\title{
Magnetic Properties of the Nucleon in a Uniform Background Field
}

\author{
Thomas James Primer
}

Supervisors: Derek Leinweber, Waseem Kamleh

The University of Adelaide

School of Chemistry and Physics

Discipline of Physics

June 2013 


\begin{abstract}
We present a calculation of the magnetic moment and magnetic polarisability of the nucleon. The calculation is performed using the background field method of lattice QCD. Dynamical results are from $32^{3} \times 64$ configurations with $2+1$ flavours of quark provided by the PACS-CS group through the ILDG. These lattices use a clover fermion action and Iwasaki gauge action with $\beta=1.9$ and physical lattice spacing $a=0.0907(13) \mathrm{fm}$. Quenched results come from $32^{3} \times 40$ lattices using a FLIC fermion action and Symanzik improved gauge action with $\beta=3.2$ and $a=0.127 \mathrm{fm}$.

The Landau energy is a crucial effect in the calculation of magnetic polarisabilities for charged particles. We derive the Landau levels and show their effect using examples of proton energy shifts in a background field.

Next we investigate the effects of moving the origin of the background gauge potential. This procedure looks similar to the technique of twisted boundary conditions, but we explain how for a quantised background field there is no change in the physical states, and show evidence using tree level calculations.

We present magnetic moment calculations for the proton and neutron, with a comparison between quenched and dynamical background field results as well as three point function results. We use the variational method in order to isolate excited states so that we can present results for the magnetic moment of the lowest lying odd-parity proton and neutron states.

Finally we present a calculation of the magnetic polarisability of the neutron. We investigate ways of improving the plateau behaviour of the energy shift, including the use of a variational analysis with a variety of source and sink smearings. Results are compared with experimental values.
\end{abstract}




\section{Statement of originality}

I certify that this work contains no material which has been accepted for the award of any other degree or diploma in any university or other tertiary institution in my name and, to the best of my knowledge and belief, contains no material previously published or written by another person, except where due reference has been made in the text. In addition, I certify that no part of this work will, in the future, be used in a submission in my name, for any other degree or diploma in any university or other tertiary institution without the prior approval of the University of Adelaide and where applicable, any partner institution responsible for the joint-award of this degree.

I give consent to this copy of my thesis, when deposited in the University Library, being made available for loan and photocopying, subject to the provisions of the Copyright Act 1968.

I also give permission for the digital version of my thesis to be made available on the web, via the University's digital research repository, the Library catalogue and also through web search engines, unless permission has been granted by the University to restrict access for a period of time. 


\section{Acknowledgements}

First of all I would like to thank my supervisors Derek and Waseem. Their guidence and advice has enabled me to produce a thesis I am proud of. Progress hasn't always been easy, but I have learned an amazing amount from them and it has made me a better physicist. I would also like to give special thanks to Matthias Burkardt, whose insight was crucial in getting us unstuck from the mud on a number of occassions.

Next I would like to thank all the PhD students I have shared my time with, both for the physics discussions and for the social interactions, whether at lunch or off at conferences or on an indoor soccer court. I would especially like to thank Dale and Ben, who always had time to help me track down recalcitrant seg faults and the like. This has been a really great group of people to spend five years studying with.

Finally I would like to thank my family, who have always been supportive of me. They were happy for me to persue what I was interested in and have also pushed me to be the best that I can. Thanks to them I have had countless opportunities to try and explain my work in laymans terms and what the real world significance of it is. If I ever work out an answer I'll let you know. 


\section{Contents}

1 Introduction 1

2 Quantum Chromodynamics 4

2.1 The Standard Model . . . . . . . . . . . . . . . . . . . . . 4

2.2 Gauge field theory . . . . . . . . . . . . . . . . . . 5

2.3 The quark model . . . . . . . . . . . . . . . . 6

2.4 The QCD Lagrangian . . . . . . . . . . . . . . . 7

2.5 Expectation values ................ 8

3 Lattice QCD $\quad \mathbf{1 0}$

3.1 Lattice gauge action . . . . . . . . . . . . . . . . 10

3.2 Lattice fermion action . . . . . . . . . . . . . . . . 12

3.2.1 Wilson action .................. . . . . . . . . . . . . . . 13

3.2 .2 Clover action .................. . . . 14

3.3 Correlation functions . . . . . . . . . . . . . . . . . 15

3.4 Interpolating fields . . . . . . . . . . . . . . . . . . . . . . . . . . . . . . . 17

3.5 Gauge field ensemble . . . . . . . . . . . . . . 18

4 The Background Field Method 20

4.1 Introduction . . . . . . . . . . . . . . . 20

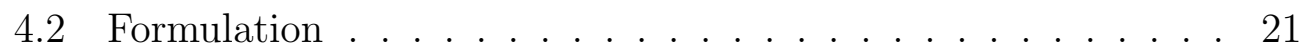

4.3 Quantising the magnetic field ............ 22

5 Simulation Details $\quad 28$

5.1 Error analysis ..................... 30

6 Landau Levels 32

6.1 Introduction . . . . . . . . . . . . . . . . . 32 
6.2 Derivation . . . . . . . . . . . . . . . . . . . 34

6.3 Examples . . . . . . . . . . . . . . . . 37

7 Twisted Boundary Conditions 43

7.1 Introduction . . . . . . . . . . . . . . . . 43

7.2 Twisted boundary conditions and momentum shifts . . . . . 45

7.2 .1 Examples . . . . . . . . . . . . . . . 46

7.3 Twist-like phases with a background field . . . . . . . . 50

7.3 .1 Explanation . . . . . . . . . . . . . . . 54

7.4 Summary . . . . . . . . . . . . . . 57

8 Magnetic Moment 58

8.1 Introduction . . . . . . . . . . . . . . . . 58

8.2 Quark model prediction . . . . . . . . . . . . . . 59

8.3 Method . . . . . . . . . . . . . . . . 61

8.4 Results . . . . . . . . . . . . . . . . . . . . . 64

8.4.1 Effective energies . . . . . . . . . . . . . . . 64

8.4.2 Magnetic field dependence . . . . . . . . . . . . . . . 68

8.4.3 Magnetic moment as a function of pion mass . . . . . . 69

8.5 Odd Parity Nucleon . . . . . . . . . . . . . . . . . 72

8.5.1 Initial results . . . . . . . . . . . . . . . 76

8.5 .2 Variational method . . . . . . . . . . . . . . . 78

8.5 .3 Results . . . . . . . . . . . . . . . . . . . 80 80

8.5.4 Effective energies . . . . . . . . . . . . . . 8 83

8.5.5 Fits to the field . . . . . . . . . . . . . . . . . 89

8.5.6 Magnetic moment as a function of pion mass . . . . . . 93

8.5.7 Quark model prediction . . . . . . . . . . . 98

9 Magnetic Polarisability 101

9.1 Introduction . . . . . . . . . . . . . . . . . . 101

9.2 Method . . . . . . . . . . . . . . . . . 102

9.3 Results . . . . . . . . . . . . . . . . . . . . . . 104

9.3.1 Effective energies . . . . . . . . . . . . . . . . 104

$9.3 .2 \quad$ Source smearing . . . . . . . . . . . . . . . . . . . . 104

9.3.3 Magnetic field dependence . . . . . . . . . . . . . . . 113

9.3.4 Magnetic polarisability as a function of pion mass . . . 114

$\begin{array}{ll}10 \text { Conclusion } & 118\end{array}$ 
$\begin{array}{lr}\text { A Quenched Results } & 126\end{array}$

A.1 Simulation details . . . . . . . . . . . . . . . 126

A.2 Magnetic moment . . . . . . . . . . . . . . . 126

A.3 Magnetic polarisability . . . . . . . . . . . . 136 


\section{List of Figures}

4.1 Top: Plaquette definition. Bottom: Background field plaquette. 24

4.2 Plaquettes at the edge of the lattice . . . . . . . . . . 25

4.3 Corner plaquette. . . . . . . . . . . . 26

6.1 Example of Landau levels from quenched calculation. . . . . . 38

6.2 Example of Landau levels from dynamical calculation. . . . . . 40

7.1 Twisted boundary effective masses at tree level. . . . . . . . . 47

7.2 Neutron effective mass twisted boundary conditions comparison. 49

7.3 Background field and twisted boundary eff. masses at tree level. 51

7.4 Energies with background field and TBCs at different smearings. 53

7.5 Example $4 \times 4$ lattice with shifted origin background field. . . . 56

8.1 Neutron effective mass plot with background field on. . . . . . 63

8.2 Spin-difference effective energy plot at $\kappa=0.13700$. . . . . . . 64

8.3 Spin-difference effective energy plot at $\kappa=0.13727$. . . . . . 65

8.4 Spin-difference effective energy plot at $\kappa=0.13754$. . . . . . 66

8.5 Spin-difference effective energy plot at $\kappa=0.13770$. . . . . . . 67

8.6 Neutron spin-difference mass shift vs field strength. . . . . . . 70

8.7 Proton spin-difference mass shift vs field strength. . . . . . . . 71

8.8 Proton magnetic moment as a function of pion mass squared. . 73

8.9 Neutron magnetic moment as a function of pion mass squared. 74

8.10 Odd parity proton spin-difference energy shift. . . . . . . . . 77

8.11 Odd parity neutron spin-difference energy shift. . . . . . . . . 77

8.12 Eigenvector values from the odd-parity variational analysis. . . 82

8.13 Projected spin-down effective mass for the odd-parity neutron. 84

8.14 Odd parity proton spin-difference energy shift at $\kappa=0.13700 . \quad 85$

8.15 Odd parity proton spin-difference energy shift at $\kappa=0.13727 .86$

8.16 Odd parity proton spin-difference energy shift at $\kappa=0.13754 . \quad 87$ 
8.17 Odd parity proton spin-difference energy shift at $\kappa=0.13770 . \quad 88$

8.18 Odd parity neutron spin-difference energy shift at $\kappa=0.13700 .89$

8.19 Odd parity neutron spin-difference energy shift at $\kappa=0.13727 .90$

8.20 Odd parity neutron spin-difference energy shift at $\kappa=0.13754$. 91

8.21 Odd parity neutron spin-difference energy shift at $\kappa=0.13770 .92$

8.22 Odd parity proton spin-diff. mass shift vs field strength. . . . 94

8.23 Odd parity neutron spin-diff. mass shift vs field strength. . . . 95

8.24 Magnetic moment of the proton and its odd-parity excitations. 96

8.25 Magnetic moment of the neutron and its odd-parity excitations. 97

9.1 Spin-averaged neutron effective mass including bare mass. . . 103

9.2 Spin-averaged energy shift at $\kappa=0.13700$. . . . . . . . . . 105

9.3 Spin-averaged energy shift at $\kappa=0.13727$. . . . . . . . . . . 106

9.4 Spin-averaged energy shift at $\kappa=0.13754$. . . . . . . . . . . 107

9.5 Spin-averaged energy shift at $\kappa=0.13770$. . . . . . . . . . . 108

9.6 Proton spin-averaged energy shift at $\kappa=0.13700$. . . . . . . 109

9.7 Spin-up and spin-down energy shifts at different smearings. . . 110

9.8 Spin-averaged energy shift with combined smearings. . . . . . 111

9.9 Spin-averaged energy shift from variational analysis. . . . . . . 112

9.10 Spin-averaged mass shift vs field strength. . . . . . . . . . 115

9.11 Neutron magnetic polarisability as a function of $m_{\pi}^{2}$. . . . . 116

A.1 Quenched spin-difference energy shift at $m_{\pi}=840 \mathrm{MeV}$. . . 128

A.2 Quenched spin-difference energy shift at $m_{\pi}=775 \mathrm{MeV}$. . . . 128

A.3 Quenched spin-difference energy shift at $m_{\pi}=693 \mathrm{MeV}$. . . . 129

A.4 Quenched spin-difference energy shift at $m_{\pi}=626 \mathrm{MeV}$. . . . 129

A.5 Quenched spin-difference energy shift at $m_{\pi}=540 \mathrm{MeV}$. . . . 130

A.6 Quenched spin-difference energy shift at $m_{\pi}=435 \mathrm{MeV}$. . . . 130

A.7 Quenched spin-difference energy shift at $m_{\pi}=275 \mathrm{MeV}$. . . . 131

A.8 Proton spin-difference energy shift vs field strength. . . . . . . 132

A.9 Neutron spin-difference energy shift vs field strength. . . . . . 133

A.10 Quenched proton magnetic moment as a function of $m_{\pi}^{2}$. . . . 134

A.11 Quenched neutron magnetic moment as a function of $m_{\pi}^{2}$. . . 135

A.12 Quenched spin-average energy shift at $m_{\pi}=840 \mathrm{MeV}$. . . . . 137

A.13 Quenched spin-average energy shift at $m_{\pi}=775 \mathrm{MeV}$. . . . . 137

A.14 Quenched spin-average energy shift at $m_{\pi}=693 \mathrm{MeV}$. . . . . 138

A.15 Quenched spin-average energy shift at $m_{\pi}=626 \mathrm{MeV}$. . . . 138

A.16 Quenched spin-average energy shift at $m_{\pi}=540 \mathrm{MeV}$. . . . 139 
A.17 Quenched spin-average energy shift at $m_{\pi}=435 \mathrm{MeV} . \quad \ldots 139$

A.18 Quenched spin-average energy shift at $m_{\pi}=275 \mathrm{MeV}$. . . 140

A.19 Neutron spin-average energy shift vs field strength. . . . . . . 141 


\section{List of Tables}

5.1 Simulation details. . . . . . . . . . . . . . . . . 28

8.1 Neutron magnetic moment values for different fit windows. . . 68

8.2 Neutron magnetic moment values from 1 and 2 parameter fits. 70

8.3 Magnetic moment values for the proton and neutron. . . . . . 73

8.4 Magnetic moment values for odd-parity states. . . . . . . . . 100

9.1 Neutron magnetic polarisability values from 1 and 2 parameter fits. . . . . . . . . . . . . . . . . . 114 


\section{Chapter 1}

\section{Introduction}

The Standard Model of particle physics has been an incredibly successful theory for describing much of the physical world. The bulk of everyday matter is made up of protons and neutrons. These in turn are formed from the combination of quarks and gluons under the part of the Standard Model known as quantum chromodynamics (QCD). In order to learn more about the properties of these particles and how they come to be, we must calculate them from the first principles of QCD. The only way to solve QCD at low energies is via the lattice.

Lattice QCD is a technique for simulating QCD interactions on a discretised space-time grid. Vast computational resources are needed to calculate physical observables to a reasonable precision. As computer power increases over time we are able to perform more precise calculations with more accurate parameters. Algorithms are also being improved all the time, decreasing errors and allowing us to reach ever closer to physical parameters. Lattice calculations allow us to test the standard model by comparing lattice results with physical results measured by experiment. As lattice results improve we also have the ability to make real predictions for many observables. In Chapters 2 and 3 we visit some of the key points in the overview of quantum chromodynamics and how calculations are implemented on the lattice. We provide citations to references which present the full details.

One interesting set of properties is the electromagnetic structure of hadrons. These values describe how hadrons interact with EM fields and require an understanding of both QCD and QED to calculate. In this work we calculate the magnetic moment and magnetic polarisability of the proton and neutron. These are fundamental properties which describe the response of the particle 
to an applied static magnetic field.

In order to calculate these properties on the lattice we use the background field method. This is a technique which simulates a classical electromagnetic field across the whole lattice. We are able to create a constant magnetic field which allows us to access the magnetic moment and polarisability via the shift in the energy due to the field. Formulating the background field method on a lattice with periodic boundary conditions requires a quantisation condition to produce a uniform magnetic field. Previous background field method results have been from lattices which were too small for the quantisation condition to be used in the calculation. In these cases the quantisation condition was avoided through various means which introduced errors in the calculation. The results for the magnetic polarisability in this work are the first ones calculated on a periodic lattice with a uniform background field everywhere. Chapter 4 describes the formulation of the background field method, with particular attention paid to the crucial quantisation condition.

In order to perform accurate background field method calculations we need a strong understanding of the issues which can arise. We have investigated the Landau levels, an effect due to the quantisation of orbital angular momentum for a charged particle in a magnetic field. This effect is very important in the calculation of the magnetic polarisability of a charged particle and as a result we do not report polarisability values for the proton in this work.

Another issue is the choice of origin for the gauge potential which creates the background field. Moving the origin is equivalent to multiplying each gauge link by a constant phase. This is closely linked to the lattice technique of twisted boundary conditions. Although one might expect moving the origin to therefore change the energy levels, we show that only the relative coupling of states is affected, and explain why this is so.

Having covered these concerns we present our calculation of the magnetic moment of the proton and neutron in Chapter 8. We have results from both dynamical and quenched configurations, with the details of the quenched calculation being found in Appendix A. Using the variational method we are also able to calculate magnetic moments for the lowest lying odd-parity states of the proton and neutron. We perform a chiral extrapolation of our results to compare with experiment.

Finally we present our calculation of the magnetic polarisability of the neutron in Chapter 9. The polarisability calculations prove much less straight forward than the moment. We investigate how the results may be improved 
through the use of different smeared sources. Again we have results from both dynamical and quenched configurations which are compared with experiment. 


\section{Chapter 2}

\section{Quantum Chromodynamics}

\subsection{The Standard Model}

The Standard Model represents our best understanding of three of the four fundamental forces that shape our universe [1]. These forces are electromagnetism, the weak interaction and the strong interaction. Electromagnetism is experienced by particles carrying electric charge and is mediated by photons, which are chargeless and massless. The weak force acts on the property of weak isospin and is carried by massive bosons, the charged $W^{+}$, and $W^{-}$ and the neutral $Z^{0}$. At high energies the electromagnetic force and the weak force become unified into a single force called the electroweak force.

The third force described by the standard model is the strong interaction [1]. The strong interaction is felt by particles which have colour charge and the associated force carrying bosons are called gluons. The only particles that carry colour charge are quarks and gluons. The name "colour" comes from the fact that three different colours combine to make a colour neutral particle in the same way that different colours of light combine to produce white light. The charges are called red, green and blue for quarks and antired, anti-green and anti-blue for anti-quarks. Each gluon carries both colour and anti-colour and has zero mass. Quarks are fermions and come in six types, called "flavours", which are arranged into three generations. There are also three generations of leptons each with a charged lepton and a neutrino. These six flavours of quark and six leptons combined with the force carrying bosons and the Higgs represent all of the known fundamental particles. With the recent discovery of the Higgs boson [2,3], all of the fundamental particles 
of the Standard Model have been observed experimentally.

Quarks and gluons combine through the strong force to create composite particles called hadrons. When a quark and an anti-quark combine they create a bosonic particle called a meson. The lightest mesons are the pions, which are formed from the combination of up and down quarks with their anti-particles. Pions are important in describing low-energy properties of other particles because virtual pion loops are readily formed, creating a "pion cloud" which surrounds the particle. When three quarks combine they form a fermionic particle called a baryon. The most common baryons are the proton and neutron, which make up the vast majority of everyday matter. There are also theorised types of particles such as tetraquarks and pentaquarks which are made from four and five quarks and anti-quarks respectively, and glueballs which are composed entirely of gluons.

\subsection{Gauge field theory}

Each of the forces described by the Standard Model is formulated as a gauge field theory [1]. Gauge field theory requires that the interactions of the fundamental particles remain invariant under local symmetry transformations of a gauge group. The combined gauge group of the Standard Model is $U(1) \times S U(2) \times S U(3)$. The groups $U(1)_{\text {weak hypercharge }}$ and $S U(2)_{\text {weak isospin }}$ were combined into the unified electroweak group by Glashow, Weinberg and Salam, for which they received the Nobel Prize in 1979.

The gauge field theory describing the strong interaction is called quantum chromodynamics (QCD) [1]. The symmetry group of QCD is $S U(3)_{\text {colour }}$. $S U(3)$ is composed of all complex $3 \times 3$ matrices that are unitary ( $U^{\dagger} U=$ $U U^{\dagger}=\mathbb{I}$ ) and have a determinant of 1 . This group is non-abelian, meaning that elements in the group do not commute, i.e. in general,

$$
[A, B] \equiv A B-B A \neq 0
$$

It is the structure of the gauge symmetry group that leads to many of the properties of QCD.

Two of the most important properties of QCD which were recognised early on are confinement and asymptotic freedom. Confinement refers to the fact that we never see isolated quarks in nature. It comes about because the potential energy between the quark anti-quark pair of a meson increases linearly as the distance between them increases. This so called "string" 
breaks when the energy is large enough to produce a quark anti-quark pair out of the vacuum. These newly created quarks combine with the existing ones to form two mesons. Similarly, when one of the quarks in a baryon is pulled away a quark anti-quark pair is produced which combines with the existing quarks to form a meson and a baryon. Since confinement can not be shown analytically, the ability to reproduce and investigate this behaviour is a major advantage and proof of concept for lattice QCD.

Asymptotic freedom [1] refers to the fact that at high energies (or equivalently, small distances) the effective coupling constant of the strong interaction approaches zero. This means that high energy probes of hadron structure should be able to detect quarks which are approximately free. As a consequence of asymptotic freedom it is possible to calculate some aspects of QCD perturbatively, but only in high energy processes where the effective coupling is small.

\subsection{The quark model}

Before the idea that particles like the nucleon were made up of smaller elementary particles, it was noticed that hadrons could be organised into families with underlying internal symmetries. Murray Gell-Mann (who also coined the term quark) came up with a system for organising particles called "the Eightfold Way". Under this system, baryons and mesons are collected into octets in which their position describes the electric charge of the particle as well as a property called "strangeness". The same principle can be applied to the spin-3/2 baryons to give us the baryon decuplet. This approach was able to predict the discovery of the $\Omega^{-}$baryon.

The term strangeness came about because certain particles such as kaons were seen to decay much more slowly than expected, which was considered strange behaviour [4]. Once the quark model was developed it was understood that the reason for this behaviour was that those particles contained a strange quark such that they could only decay via weak interactions, which are slower than strong decays. This is where the strange quark gets its name.

The structure of these multiplets arises due to an internal symmetry of quarks called $S U(3)_{\text {flavour }}$ symmetry. This symmetry relies on the fact that the masses of the up, down and strange quark are quite similar, at least when compared to the masses of the hadrons they comprise. The symmetry is broken however due to the fact that the strange quark mass is around ten 
times that of the up and down quarks. This mass difference leads to the difference in masses of the members of each multiplet.

It was originally believed that flavour symmetry was the fundamental symmetry of QCD. This was disproved when a new particle was discovered, the $\Delta^{++}$, which is made up of three up quarks which are all spin-up [4]. Since this is forbidden by the Pauli exclusion principle there needed to be another internal property to separate the quarks, and colour was invented.

\subsection{The QCD Lagrangian}

In quantum field theory we use the Lagrangian formalism for describing systems and calculating their equations of motion. The dynamical behaviour of quarks and gluons is governed by the QCD Lagrangian,

$$
\mathcal{L}_{Q C D}=\bar{\psi}(x)\left(i \gamma^{\mu} D_{\mu}-m\right) \psi(x)-\frac{1}{2} \operatorname{Tr}\left(F_{\mu \nu} F^{\mu \nu}\right),
$$

where $\psi, \bar{\psi}$ are four-component Dirac spinors representing the quark fields, $\gamma^{\mu}$ are the $\gamma$-matrices in the Dirac representation [5], and $D_{\mu}$ is the covariant derivative

$$
D_{\mu} \equiv \partial_{\mu}+i g A_{\mu}
$$

The field strength tensor, $F_{\mu \nu}$ is defined by,

$$
F_{\mu \nu}=\partial_{\mu} A_{\nu}-\partial_{\nu} A_{\mu}+i g\left[A_{\mu}, A_{\nu}\right]
$$

The gauge fields, and therefore the field strength tensor, can be linearly combined from the generators of $S U(3)$,

$$
A_{\mu}(x)=\sum_{a=1}^{8} \frac{\lambda^{a}}{2} A_{\mu}^{a}(x), \quad F_{\mu \nu}(x)=\sum_{a=1}^{8} \frac{\lambda^{a}}{2} F_{\mu \nu}^{a}(x),
$$

where $\lambda^{a}$ are the generators of $S U(3)$. They are represented by $3 \times 3$ traceless Hermitian matrices satisfying $\operatorname{Tr}\left(\lambda^{a} \lambda^{b}\right)=\delta^{a b}$ and $\left[\frac{\lambda^{a}}{2}, \frac{\lambda^{b}}{2}\right]=i f^{a b c} \frac{\lambda^{c}}{2}$, where $f^{a b c}$ are the antisymmetric structure constants. There are eight generators, corresponding to eight different types of gluon.

Due to the non-abelian nature of $S U(3)$, the $F^{2}$ term in the Lagrangian gives rise to $A^{3}$ and $A^{4}$ terms which desribe 3 and 4 gluon vertices. This 
means that gluons carry colour charge and can interact with each other, which is a major difference between QCD and QED.

From the Lagrangian density we calculate the QCD action by taking the integral over space-time,

$$
\begin{aligned}
S_{Q C D} & =\int d^{4} x \mathcal{L}_{Q C D}\left(\psi, \bar{\psi}, A_{\mu}\right) \\
& =\int d^{4} x \bar{\psi}(x)\left(i \gamma^{\mu} D_{\mu}-m\right) \psi(x)-\frac{1}{2} \int d^{4} x \operatorname{Tr}\left(F_{\mu \nu} F^{\mu \nu}\right) \\
& =S_{F}+S_{G},
\end{aligned}
$$

where we have broken the action into a fermionic part and a gluonic part.

The QCD Lagrangian and action must be invariant under both global and local $S U(3)$ transformations. i.e. for local transformations,

$$
\begin{aligned}
\psi(x) & \rightarrow G(x) \psi(x) \\
\psi \overline{(x)} & \rightarrow \psi \overline{(x) G(x)} \\
A_{\mu}(x) & \rightarrow G(x) A_{\mu}(x) G^{-1}(x)-\frac{i}{g} G(x) \partial_{\mu}\left(G^{-1}(x)\right),
\end{aligned}
$$

where $G(x)=\exp \left(i \omega^{a}(x) \lambda_{a}\right)$. These symmetries govern the interactions of $\mathrm{QCD}$ and give rise to the conservation of colour charge.

\subsection{Expectation values}

We calculate observables in QCD from vacuum expectation values following the Feynman path integral approach [1]. We begin with the Euclidean spacetime partition function, also known as the vacuum-to-vacuum amplitude, which is written as,

$$
Z=\int \mathcal{D} A_{\mu} \mathcal{D} \psi \mathcal{D} \bar{\psi} e^{-S}
$$

where $A_{\mu}$ is the gauge field and $\psi$ and $\bar{\psi}$ are fermion fields which are represented by Grassman variables. $S$ is the QCD action, given by,

$$
S=\int d^{4} x\left(\frac{1}{2} \operatorname{Tr} F_{\mu \nu} F^{\mu \nu}-\bar{\psi} M \psi\right),
$$

where $\mathrm{M}$ is the Dirac operator. To calculate the partition function we take advantage of the Grassman algebra [1], which describes the anti-commuting 
fermion fields, $\left\{\psi_{\mu}, \psi_{\nu}\right\}=0$. This allows us to integrate out the fermion fields exactly, leaving us with,

$$
Z=\int \mathcal{D} A_{\mu} \operatorname{det} M e^{\int d^{4} x\left(-\frac{1}{2} \operatorname{Tr} F_{\mu \nu} F^{\mu \nu}\right)}
$$

which is an integral only over gauge configurations.

In order to obtain results for physical observables we calculate vacuum expectation values

$$
\langle\hat{O}\rangle=\langle\Omega|\hat{O}| \Omega\rangle=\frac{1}{Z} \int \mathcal{D} A_{\mu} \hat{O} \operatorname{det} M e^{-S_{G}},
$$

where $\hat{O}$ is some operator or combination of operators that we are interested in. $\int \mathcal{D} A_{\mu}$ indicates a functional integral over all of the infinite possible configurations of the gluon field. $\operatorname{det} M e^{-S_{G}}$ is a weighting for each gauge configuration, where $S_{G}$ is the gauge action. The result is normalised by the partition function. 


\section{Chapter 3}

\section{Lattice QCD}

Problems in QED are generally solved using a perturbative expansion in the coupling. This is possible due to the fact that the coupling is small, so that higher order terms quickly become negligible. In QCD at large energies we have asymptotic freedom so the coupling becomes small and perturbation theory can be used. At lower energy scales however the dynamics of QCD give rise to a coupling that is large and a non-trivial vacuum structure. Thus a non-perturbative approach is required.

\subsection{Lattice gauge action}

Lattice gauge theory was developed by Wilson [6] as a method for solving QCD non-perturbatively. In lattice theory we discretise space-time into a Euclidean hypercube lattice with finite volume $V$ and lattice spacing $a$. In the standard formalism the quark fields $\psi(x)$ exist at the lattice sites and the gauge fields are represented by links from each lattice site to each of its neighbours in the form of $S U(3)$ colour matrices. Derivatives are replaced with finite differences and integrals with discrete sums. The definition of the gauge links is given by the path ordered integral,

$$
U_{\mu}(x)=\mathcal{P} \exp \left(-i g \int_{0}^{a} d \lambda A_{\mu}(x+\lambda \hat{\mu})\right),
$$

where the $\mu$ index labels the direction from the lattice site at $x$ to the site at $x+a \hat{\mu}, a$ is the lattice spacing and $g$ is the QCD coupling constant. For a sufficiently smooth gauge field and small lattice spacing this can be 
approximated as,

$$
U_{\mu}(x) \simeq e^{-i g a A_{\mu}(x)} .
$$

The transformation property of the fields under a general local gauge transformation $\Lambda(X)$ is given by,

$$
\psi(x) \rightarrow \Lambda(x) \psi(x), \quad U_{\mu}(x) \rightarrow \Lambda(x) U_{\mu}(x) \Lambda^{\dagger}(x+\hat{\mu}) .
$$

The product of gauge links around a closed path is called a Wilson loop. The smallest such loop is a square called a plaquette [1],

$$
P_{\mu \nu}(x)=\left(U_{\mu}(x) U_{\nu}(x+\hat{\mu}) U_{\mu}^{\dagger}(x+\hat{\nu}) U_{\nu}^{\dagger}(x)\right),
$$

Using the unitarity of the gauge transformation $\left(\Lambda \Lambda^{\dagger}=\Lambda^{\dagger} \Lambda=1\right)$ and the cyclic property of the trace it is easy to see that the plaquette is gauge invariant. We can write the plaquette more explicitly as,

$$
P_{\mu \nu}(x)=e^{-i g a A_{\mu}(x)} e^{-i g a A_{\nu}(x+\mu)} e^{i g a A_{\mu}(x+\nu)} e^{i g a A_{\nu}(x)},
$$

Using the Baker-Campbell-Hausdorff formula for non-commuting operators this can be written as,

$$
P_{\mu \nu}(x)=e^{-i g a^{2}\left(F_{\mu \nu}+\mathcal{O}\left(a^{2}\right)\right)} .
$$

We see clearly that the field strength tensor is encoded in the plaquettes. Expanding the exponential in Eq. (3.6) gives,

$$
\begin{aligned}
P_{\mu \nu}(x) & =1-i g a^{2} F_{\mu \nu}+\frac{1}{2} g^{2} a^{4} F_{\mu \nu} F^{\mu \nu}+\mathcal{O}\left(a^{4}\right) \\
1-\frac{1}{3} \mathcal{R} e \operatorname{Tr}\left[P_{\mu \nu}(x)\right] & =a^{4} \frac{g^{2}}{6}\left(\operatorname{Tr} F_{\mu \nu} F^{\mu \nu}+\mathcal{O}\left(a^{2}\right)\right)
\end{aligned}
$$

Using the definition of the plaquette in Eq. (3.8) it is possible to define what is known as the Wilson gluon action,

$$
S_{\mathrm{W}}=\beta \sum_{x} \sum_{\mu>\nu}\left(1-\frac{1}{3} \mathcal{R} e \operatorname{Tr}\left[P_{\mu \nu}(x)\right]\right) .
$$

In the continuum limit where the lattice spacing $a \rightarrow 0$ we have,

$$
\begin{aligned}
\sum_{x} & \rightarrow \frac{1}{a^{4}} \int d^{4} x, \\
\mathcal{O}\left(a^{2}\right) & \rightarrow 0, \\
S_{\mathrm{W}} & \rightarrow \beta \frac{g^{2}}{6} \int d^{4} x \frac{1}{2} \operatorname{Tr} F_{\mu \nu} F^{\mu \nu} .
\end{aligned}
$$


Therefore to match the continuum action we require that $\beta=6 / g^{2}$. The errors in the Wilson gluon action are $\mathcal{O}\left(a^{2}\right)$ and $\mathcal{O}\left(g^{2} a^{2}\right)$. It is possible to remove the $a^{2}$ errors using other Wilson loops. We use $2 \times 1$ and $1 \times 2$ rectangular loops, which have different $\mathcal{O}\left(a^{2}\right)$ errors that cancel with those in $P_{\mu \nu}$ to give an improved action,

$$
\begin{aligned}
S_{\text {Imp }} & =\beta \sum_{x} \sum_{\mu>\nu}\left[\frac{5}{3}\left(1-P_{\mu \nu}\right)-\frac{1}{12}\left(1-R_{\mu \nu}^{1 \times 2}\right)+\frac{1}{12}\left(1-R_{\nu \mu}^{2 \times 1}\right)\right] \\
& =a^{4} \sum_{x} \sum_{\mu>\nu}\left[\frac{1}{4} F_{\mu \nu} F^{\mu \nu}+\mathcal{O}\left(a^{4}\right)+\mathcal{O}\left(g^{2} a^{2}\right)\right]
\end{aligned}
$$

This improved action has errors which are $\mathcal{O}\left(a^{4}\right)$ and $\mathcal{O}\left(g^{2} a^{2}\right)$. There are several methods for calculating perturbative corrections to the coefficients of the plaquette and rectangle terms. Mean field complements the two extra links in $R_{\mu \nu}$ with $1 / u_{0}^{2}$ where $u_{0}$ is the mean link, $u_{0}=\left\langle\frac{1}{4} P_{\mu \nu}\right\rangle$. An alternative approach uses the renormalization group transformations to determine the corrections. The Iwasaki action is one such, given by [1],

$$
S_{\text {Iwasaki }}=\beta \sum_{x} \sum_{\mu, \nu}\left(P_{\mu \nu}-0.0907 R_{\mu \nu}^{1 \times 2}\right) .
$$

\subsection{Lattice fermion action}

After the gauge action the next step is to discretise the quark part of the QCD action on the lattice. From the QCD Lagrangian we obtain the Euclidean space-time continuum Dirac fermion action,

$$
\bar{\psi}\left(\gamma^{\mu} D_{\mu}+m\right) \psi
$$

where $D_{\mu}$ is the matrix form of the covariant derivative from Eq. (2.3). Wilson discretised this action by simply replacing the derivatives with a central difference formula and representing the gluon fields by the previously defined link variables,

$$
\bar{\psi} \gamma^{\mu} D_{\mu} \psi=\frac{1}{2 a} \bar{\psi}(x) \sum_{\mu} \gamma^{\mu}\left[U_{\mu}(x) \psi(x+\hat{\mu})-U_{\mu}^{\dagger}(x-\hat{\mu}) \psi(x-\hat{\mu})\right] .
$$

By Taylor expanding $U_{\mu}$ and $\psi$ and taking the limit $a \rightarrow 0$ the continuum equation is recovered as required for physical consistency. Using this definition of the covariant derivative we have the simplest possible lattice fermion 
action, known as the "naive" fermion action,

$$
\begin{aligned}
S_{N}= & m_{q} \sum_{x} \bar{\psi}(x) \psi(x) \\
& +\frac{1}{2 a} \sum_{x, \mu} \bar{\psi}(x) \gamma^{\mu}\left[U_{\mu}(x) \psi(x+\hat{\mu})-U_{\mu}^{\dagger}(x-\hat{\mu}) \psi(x-\hat{\mu})\right] \\
= & \sum_{x} \bar{\psi}(x) M_{x y}^{N}[U] \psi(y)
\end{aligned}
$$

where $M^{N}$ is the Dirac operator interaction matrix and $\gamma^{\mu}$ are the gamma matrices given in the Dirac representation. The Taylor expansion shows that the error in the naive action is $\mathcal{O}\left(a^{2}\right)$.

The problem with the naive fermion action is that it gives rise to 16 flavours of quarks. This can be seen by taking the inverse of the free field propagator, obtained from the Fourier transform of the action with $U_{\mu}=1$ for all gauge links,

$$
S^{-1}(p)=m_{q}+\frac{i}{a} \sum_{\mu} \gamma^{\mu} \sin p_{\mu} a
$$

which has 16 zeros within the Brillouin cell, $\frac{-\pi}{a}<p_{\mu} \leqslant \frac{\pi}{a}$, in the limit $m_{q} \rightarrow 0$. This is known as the fermion doubling problem and makes the naive fermion action unacceptable.

\subsubsection{Wilson action}

Wilson solved the fermion doubling problem by adding an irrelevant dimension five operator called the "Wilson term" to the action. The operator is "irrelevant" because it is proportional to $a$ and therefore vanishes in the 
continuum limit. This gives the action known as the Wilson action,

$$
\begin{aligned}
S_{W}= & m_{q} \sum_{x} \bar{\psi}(x) \psi(x) \\
& +\frac{1}{2 a} \sum_{x, \mu} \bar{\psi}(x) \gamma^{\mu}\left[U_{\mu}(x) \psi(x+\hat{\mu})-U_{\mu}^{\dagger}(x-\hat{\mu}) \psi(x-\hat{\mu})\right] \\
& -\frac{r}{2 a} \sum_{x, \mu} \bar{\psi}\left[U_{\mu}(x) \psi(x+\hat{\mu})-2 \psi(x)+U_{\mu}^{\dagger}(x-\hat{\mu}) \psi(x-\hat{\mu})\right] \quad(3.17) \\
= & \frac{m_{q} a+4 r}{a} \sum_{x} \bar{\psi}(x) \psi(x) \\
& +\frac{1}{2 a} \sum_{x, \mu} \bar{\psi}(x)\left[\left(\gamma^{\mu}-r\right) U_{\mu}(x) \psi(x+\hat{\mu})-\left(\gamma^{\mu}+r\right) U_{\mu}^{\dagger}(x-\hat{\mu}) \psi(x-\hat{\mu})\right] \\
\equiv & \sum_{x, y} \bar{\psi}_{x}^{L} M_{x y}^{W} \psi_{y}^{L},
\end{aligned}
$$

where the interaction matrix $M^{W}$ is written as,

$$
a M_{x y}^{W}[U]=\delta_{x y}-\kappa \sum_{\mu}\left[\left(r-\gamma^{\mu}\right) U_{x, \mu} \delta_{x, y-\mu}+\left(r+\gamma^{\mu}\right) U_{x-\mu, \mu}^{\dagger} \delta_{x, y+\mu}\right]
$$

with the rescaling,

$$
\begin{aligned}
\kappa & =1 /\left(2 m_{q} a+8 r\right) \\
\psi^{L} & =\sqrt{m_{q} a+4 r} \psi=\psi / \sqrt{2 \kappa} .
\end{aligned}
$$

Here $r$ is what is known as the Wilson parameter and is almost always given the value $r=1$. The addition of this term suppresses the 15 extra flavours of quark by giving them a mass proportional to $r / a$. Unfortunately the Wilson action has $\mathcal{O}(a)$ errors and breaks chiral symmetry.

\subsubsection{Clover action}

In order to improve upon the $\mathcal{O}(a)$ errors of the Wilson action, Sheikholeslami and Wohlert [7] added another irrelevant operator term of dimension 5, called the "clover" term. This removes the $\mathcal{O}(a)$ errors without ruining Wilson's fix of the doubling problem. The resulting Sheikholeslami-Wohlert (clover) action is,

$$
S_{S W}=S_{W}-\frac{i a C_{S W} \kappa r}{4} \bar{\psi}(x) \sigma_{\mu \nu} F_{\mu \nu} \psi(x),
$$


where $\sigma_{\mu \nu}=\frac{i}{2}\left[\gamma_{\mu}, \gamma_{\nu}\right]$. The clover coefficient, $C_{S W}=1$ at tree level, must be tuned to effectively remove $\mathcal{O}(a)$ errors. The most common method is non-perturbative improvement [8]. The SW action is known as the clover action because the field strength tensor in the term is calculated from the four plaquettes surrounding a point on the lattice in the $\mu \nu$ plane, which make a shape like a four leaf clover. Using the previous definition of the plaquette from Eq. (3.8) we get an expression for the field strength tensor,

$$
g a^{2} F_{\mu \nu}=\frac{-i}{2}\left[O_{\mu \nu}^{(1)}-O_{\mu \nu}^{(1) \dagger}-\frac{1}{3} \operatorname{Tr}\left(O_{\mu \nu}^{(1)}-O_{\mu \nu}^{(1) \dagger}\right)\right],
$$

where $O_{\mu \nu}^{(1)}$ is the sum of the four plaquettes in the $\mu \nu$ plane around $x$ and $1 / 3$ of the trace is subtracted to make $F_{\mu \nu}$ traceless. Using this definition of the field strength tensor with the SW action gives us a fermion action accurate to $\mathcal{O}\left(a^{2}\right)$.

\subsection{Correlation functions}

Recall the expression for the expectation value of an observable,

$$
\langle\hat{O}\rangle=\langle\Omega|\hat{O}| \Omega\rangle=\frac{1}{Z} \int \mathcal{D} A_{\mu} \hat{O} \operatorname{det} M e^{-S_{G}} .
$$

An important such expectation value on the lattice is the two point correlation function. Here we are interested in baryon energies so we use baryon interpolating functions as our operators,

$$
G_{2}(t, \mathbf{p})=\sum_{\mathbf{x}} e^{-i \mathbf{p} \cdot \mathbf{x}}\left\langle\Omega\left|\chi_{B}(x) \bar{\chi}_{B}(0)\right| \Omega\right\rangle .
$$

This describes the amplitude for a baryon being created at zero and then annihilated at space-time point $x .|\Omega\rangle$ is the non-trivial QCD vacuum state. We insert a complete set of Hamiltonian eigenstates and note the interpolating field couples to all states with the correct quantum numbers,

$$
G_{2}(t, \mathbf{p})=\sum_{B, s, \mathbf{q}} \sum_{\mathbf{x}} e^{-i \mathbf{p} \cdot \mathbf{x}}\left\langle\Omega\left|\chi_{B}(x)\right| B, s, \mathbf{q}\right\rangle\left\langle B, s, \mathbf{q}\left|\bar{\chi}_{B}(0)\right| \Omega\right\rangle,
$$

We assume a wall boundary condition in time such that states do not traverse the boundary. The translation operator $e^{-i \hat{P} \cdot \mathbf{x}}$, where $\hat{P}$ is the four momentum operator, is applied so that the creation and annihilation operators act 
at the same point,

$$
\begin{aligned}
G_{2}(t, \mathbf{p}) & =\sum_{B, s, \mathbf{q}} \sum_{\mathbf{x}} e^{-i \mathbf{p} \cdot \mathbf{x}}\left\langle\Omega\left|e^{+i \hat{P} \cdot \mathbf{x}} \chi_{B}(0) e^{-i \hat{P} \cdot \mathbf{x}}\right| B, s, \mathbf{q}\right\rangle\left\langle B, s, \mathbf{q}\left|\bar{\chi}_{B}(0)\right| \Omega\right\rangle \\
& =\sum_{B, s, \mathbf{q}} e^{-i E_{B} t} \sum_{\mathbf{x}} e^{-i(\mathbf{p}-\mathbf{q}) \cdot \mathbf{x}}\left\langle\Omega\left|\chi_{B}(0)\right| B, s, \mathbf{q}\right\rangle\left\langle B, s, \mathbf{q}\left|\bar{\chi}_{B}(0)\right| \Omega\right\rangle \\
& =\sum_{B, s, \mathbf{q}} e^{-i E_{B} t} \delta_{\mathbf{p q}}\left\langle\Omega\left|\chi_{B}(0)\right| B, s, \mathbf{q}\right\rangle\left\langle B, s, \mathbf{q}\left|\bar{\chi}_{B}(0)\right| \Omega\right\rangle \\
& =\sum_{B} e^{-i E_{B} t} \sum_{s}\left\langle\Omega\left|\chi_{B}(0)\right| B, s, \mathbf{p}\right\rangle\left\langle B, s, \mathbf{p}\left|\bar{\chi}_{B}(0)\right| \Omega\right\rangle
\end{aligned}
$$

then we define,

$$
\begin{aligned}
\left\langle\Omega\left|\chi_{B}\right| B, s, \mathbf{q}\right\rangle & =\lambda_{B, \mathbf{q}} \psi_{B}^{s} \\
\left\langle B, s, \mathbf{q}\left|\bar{\chi}_{B}\right| \Omega\right\rangle & =\bar{\lambda}_{B, \mathbf{q}} \bar{\psi}_{B}^{s}
\end{aligned}
$$

where $\psi_{B}^{s}, \bar{\psi}_{B}^{s}$ are Dirac spinors, $\psi_{B}^{s}=\sqrt{\frac{M_{B}}{E_{B}}} u(\mathbf{p}, s)$, and $\lambda, \bar{\lambda}$ are the couplings of the interpolators at the sink and source. We can then write the correlation function as,

$$
G_{2}(t, \mathbf{p})=\sum_{B}\left|\lambda_{B, \mathbf{p}}\right|^{2} e^{-i E_{B}(\mathbf{p}) t} \frac{M_{B}}{E_{B}} \sum_{s} u(\mathbf{p}, s) \bar{u}(\mathbf{p}, s)
$$

Recalling that lattice QCD is formulated in Euclidean space time, we replace $t \rightarrow-i t$. We project out zero momentum such that $E_{B}=M_{B}$, resulting in,

$$
G_{2}(t, \mathbf{0})=\sum_{B}\left|\lambda_{B, \mathbf{p}}\right|^{2} e^{-M_{B} t} \frac{\gamma \cdot p+M_{B}}{2 E_{B}}
$$

We can calculate a baryon effective mass via,

$$
a M(t)=\ln \left(\frac{G_{2}(\mathbf{0}, t)}{G_{2}(\mathbf{0}, t+1)}\right)
$$

where it is understood in the above formula that the appropriate element of $G_{2}(\mathbf{0}, t)$ is considered based on the desired spin and parity of the baryon. The effective mass contains contributions from all of the excited states created by the interpolator as well as the ground state. The higher energy states are exponentially suppressed such that at large times only the ground state 
contribution remains. By waiting for the effective mass to plateau and then fitting we can extract the mass of the baryon ground state.

At zero momentum the even-parity spin-up and spin-down states propagate in the $(1,1)$ and $(2,2)$ components of the Dirac matrix respectively. In standard mass calculations the spins are equivalent in the ensemble average so the average of spins can be taken to improve statistics. In our background field calculations, however, we need to take advantage of the fact that spin-up and spin-down energies are different in the presence of a magnetic field.

\subsection{Interpolating fields}

In order to actually calculate the two point baryon correlation function we express the baryon interpolating fields in terms of quark fields. The standard type 1 interpolator for the proton is written,

$$
\chi_{1}=\epsilon^{a b c}\left(u^{a T} C \gamma^{5} d^{b}\right) u^{c},
$$

where $\epsilon^{a b c}$ is the Levi-Civita tensor, $C$ is the charge conjugation matrix (= $i \gamma_{0} \gamma_{2}$ in the Dirac representation), and $u$ and $d$ are Dirac spinors representing up and down quarks respectively. $T$ indicates a transpose in the Dirac index and $a, b$, and $c$ are colour indices which are summed over. We can also define the adjoint field,

$$
\begin{aligned}
\bar{\chi}_{1}=\chi_{1}^{\dagger} \gamma^{0} & =\epsilon^{a b c} u^{a \dagger}\left(d^{b \dagger} \gamma^{5} C^{\dagger} u^{c T \dagger}\right) \\
& =\epsilon^{a b c} \bar{u}^{a}\left(\bar{d}^{b} \gamma^{0} \gamma^{5} C^{\dagger} \gamma^{0} \bar{u}^{c T}\right),
\end{aligned}
$$

where we have inserted $\left(\gamma^{0}\right)^{2}=I$ to get $\bar{u}=u^{\dagger} \gamma^{0}$ and $\bar{d}=d^{\dagger} \gamma^{0}$. Using these definitions we can then write the quark level correlation function,

$$
\begin{aligned}
G_{2 \gamma \gamma^{\prime}}(t, \mathbf{p})= & \sum_{\mathbf{x}} e^{-i \mathbf{p} \cdot \mathbf{x}}\langle\Omega| \epsilon^{a b c}\left(u_{\alpha}^{a T}(x)\left(C \gamma^{5}\right)_{\alpha \beta} d_{\beta}^{b}(x)\right) u_{\gamma}^{c}(x) \\
& \epsilon^{a^{\prime} b^{\prime} c^{\prime}} \bar{u}_{\gamma^{\prime}}^{a^{\prime}}(0)\left(\bar{d}_{\alpha^{\prime}}^{b^{\prime}}(0)\left(\gamma^{0} \gamma^{5 \dagger} C^{\dagger} \gamma^{0}\right)_{\alpha^{\prime} \beta^{\prime}} \bar{u}_{\beta^{\prime}}^{c^{\prime} T}(0)\right)|\Omega\rangle \\
= & \sum_{\mathbf{x}}-e^{-i \mathbf{p} \cdot \mathbf{x}} \epsilon^{a b c} \epsilon^{a^{\prime} b^{\prime} c^{\prime}}\left(C \gamma^{5}\right)_{\alpha \beta}\left(\gamma^{0} \gamma^{5 \dagger} C^{\dagger} \gamma^{0}\right)_{\alpha^{\prime} \beta^{\prime}} \\
& \left\langle\Omega\left|\left(u_{\alpha}^{a T}(x) d_{\beta}^{b}(x)\right) u_{\gamma}^{c}(x) \bar{u}_{\gamma^{\prime}}^{a^{\prime}}(0)\left(\bar{d}_{\alpha^{\prime}}^{b^{\prime}}(0) \bar{u}_{\beta^{\prime}}^{c^{\prime} T}(0)\right)\right| \Omega\right\rangle(3.3
\end{aligned}
$$

Here $\alpha, \beta$, and $\gamma$ and $\alpha^{\prime}, \beta^{\prime}$, and $\gamma^{\prime}$ are Dirac indices and there is a sum over $\alpha, \beta, \alpha^{\prime}$, and $\beta^{\prime}$. The Grassmann integration, cf. (2.7), demands we make 
all possible contractions between the quark fields, replacing pairs of $\psi \bar{\psi}$ with the inverse fermion matrix, $M^{-1}$, which corresponds to Wick's theorem. This gives us the correlation function in terms of quark propagators,

$$
\begin{aligned}
G_{2 \gamma \gamma^{\prime}}= & \sum_{\mathbf{x}}-e^{-i \mathbf{p} \cdot \mathbf{x}} \epsilon^{a b c} \epsilon^{a^{\prime} b^{\prime} c^{\prime}}\left(C \gamma^{5}\right)_{\alpha \beta}\left(\gamma^{0} \gamma^{5 \dagger} C^{\dagger} \gamma^{0}\right)_{\alpha^{\prime} \beta^{\prime}} \\
& \left\{S_{u, \gamma \gamma^{\prime}}^{a a^{\prime}} S_{d, \beta \alpha^{\prime}}^{b b^{\prime}} S_{u, \alpha \beta^{\prime}}^{c c^{\prime}}+S_{u, \gamma \beta^{\prime}}^{c c^{\prime}} S_{d, \beta \alpha^{\prime}}^{b b^{\prime}} S_{u, \alpha \gamma^{\prime}}^{a a^{\prime}}\right\} \\
= & \sum_{\mathbf{x}}-e^{-i \mathbf{p} \cdot \mathbf{x}} \epsilon^{a b c} \epsilon^{a^{\prime} b^{\prime} c^{\prime}}\left\{S _ { u , \gamma \gamma ^ { \prime } } ^ { c c ^ { \prime } } \operatorname { T r } \left[S_{u}^{a a^{\prime}}\left(C \gamma^{5} S_{d}^{b b^{\prime}} \gamma^{0} \gamma^{5 \dagger} C^{\dagger} \gamma^{0}\right)^{T}\right.\right. \\
& {\left.\left[S_{u}^{c c^{\prime}}\left(C \gamma^{5} S_{d}^{b b^{\prime}} \gamma^{0} \gamma^{5 \dagger} C^{\dagger} \gamma^{0}\right)^{T} S_{u}^{a a^{\prime}}\right]_{\gamma \gamma^{\prime}}\right\}, }
\end{aligned}
$$

where the Feynman propagators are defined,

$$
S_{u, \alpha \beta}^{a b}=S_{u, \alpha \beta}^{a b}(x, 0)=\langle\Omega| \text { contraction between } u_{\alpha}^{a}(x) \text { and } \bar{u}_{\beta}^{b}(0)|\Omega\rangle
$$

and similarly for $S_{d}$. The two point correlation function for the neutron is obtained the same way but with up and down quarks switched in the interpolating field. The numerical values of the Feynman propagators are calculated from the matrix inverse of the Dirac operator for the appropriate fermion action on a given background gauge field,

$$
S_{F}(y, \beta, b ; x, \alpha, a)=\left(M^{-1}\right)_{x, \alpha, a}^{y, \beta, b},
$$

which gives the amplitude for a quark to go from space-time point $x$ with Dirac index $\alpha$ and colour $a$ to point $y$ with Dirac index $\beta$ and colour $b$.

\subsection{Gauge field ensemble}

In order to get a final value for the expectation value we must perform the functional integral $\int \mathcal{D} A_{\mu}$. We cannot calculate correlation functions on an infinite number of gauge fields so we replace the functional integral with an average over a finite gauge ensemble,

$$
\langle\hat{O}\rangle \approx \frac{1}{n} \sum_{i=1}^{n} \hat{O}\left[\{U\}_{i}\right],
$$

where the configuration $\{U\}$ is selected with probability,

$$
\operatorname{det} M e^{-S_{G}}
$$


A gauge ensemble is generated using an importance sampling Monte Carlo technique. Each configuration is generated from the one before it by proposing random (or pseudo-random) updates $U \rightarrow U^{\prime}$ which are accepted with probability $P\left(U \rightarrow U^{\prime}\right)=e^{-\Delta S}$, where $\Delta S=S\left[U^{\prime}\right]-S[U]$. This results in an ensemble which is representative of the physical world.

It is possible to save a great deal of computation by simply setting $\operatorname{det} M=1$ because the remaining gluonic part of the action is entirely local. This is known as the quenched approximation and is equivalent to suppressing disconnected pion loops. The effect of the quenched approximation on observables is non-trivial. In this work we perform calculations on both quenched and dynamical gauge configurations and compare the results. 


\section{Chapter 4}

\section{The Background Field Method}

\subsection{Introduction}

The background field formalism is a well established technique for investigating electromagnetic properties in lattice QCD [9-21]. It allows us to introduce a classical electric or magnetic field over the lattice and determine hadron properties by looking at the change in energy. The technique is easy to implement computationally. Calculations proceed like any other lattice calculation but with a slight modification to the gauge field. This modification comes in the form of a $U(1)$ phase factor which multiplies the usual gauge links. By making the appropriate choice of phase factors it is possible to introduce a constant electric or magnetic field on the lattice.

We calculate propagators with a given background field and then combine them to produce hadron correlators with a magnetic field present. Unlike many lattice calculations, up and down quarks must be treated separately due to their different charges. We consider propagators in background fields related by the same factor as the relationship between their charges. Hadron energies at zero momentum are extracted in the usual way, except that the effective masses are shifted by the presence of the background field. For a constant magnetic field the relationship between the field and the effective mass of a particle of charge $e$ is given by $[9,11]$,

$$
E(B)=M-\boldsymbol{\mu} \cdot \mathbf{B}+\frac{e|B|}{2 M}-\frac{4 \pi}{2} \beta B^{2}+\mathcal{O}\left(B^{3}\right),
$$

where we define $\mu$ to be the magnetic moment, and $\beta$ to be the magnetic polarisability. The term $e|B| / 2 M$ describes the Landau energy. From this 
relation the magnetic moment and magnetic polarisability of a hadron can be determined.

The addition of a magnetic field to the lattice can have subtleties and cause additional issues which may not be immediately apparent. In Chapter 6 we discuss the Landau levels, an effect that arises due to the quantisation of orbital angular momentum, which affects charged particles. In Chapter 7 we look at twisted boundary conditions, a technique which is similar in implementation to the background field method. In particular, we explore how a shift in the origin of the background field can have unintended consequences for background field calculations.

There are also issues which are specific to electric fields. One is the realness of the phase, which is in question because electric background fields involve the zeroth (or time) dimension [20]. Another is that charged particles in electric fields are moved by the field, making their zero momentum state hard to pin down. Understanding issues like these is crucial for performing precise background field method calculations.

\subsection{Formulation}

The background field method is formulated by beginning in the continuum limit. We modify the QCD Dirac operator with the addition of a term describing minimal electromagnetic coupling.

$$
\begin{aligned}
D_{\mu} & =\partial_{\mu}+g G_{\mu} \\
\rightarrow D_{\mu}^{\prime} & =\partial_{\mu}+g G_{\mu}+q A_{\mu} .
\end{aligned}
$$

where $g$ is the QCD coupling, $G_{\mu}$ is the gluon field, $q$ is the electromagnetic coupling, and $A_{\mu}$ is the electromagnetic gauge field. The EM gauge field is then discretised in the same manner as the gluon field in Eq. (3.1),

$$
U_{\mu}^{(B)}(x)=\exp \left(i q \int_{x}^{x+a \hat{\mu}} \mathrm{d} y_{\mu} A_{\mu}(y)\right)
$$

which for an appropriately smooth, continuous gauge field results in,

$$
U_{\mu}^{(B)}(x) \approx e^{i a q A_{\mu}(x)} .
$$


This term then multiplies the normal gauge links. Therefore we can simply consider the gluon field to have been modified by a $U(1)$ phase,

$$
\begin{aligned}
U_{\mu}(x) \rightarrow U_{\mu}^{\prime}(x) & =U_{\mu}(x) U_{\mu}^{(B)}(x) \\
& =U_{\mu}(x) e^{i a q A_{\mu}(x)},
\end{aligned}
$$

and otherwise perform all calculations as usual using these modified gauge links.

\subsection{Quantising the magnetic field}

In order to perform useful calculations with the background field method we need to have a magnetic field with a well defined magnitude and direction. We use Maxwell's equations to make a choice of gauge potential which will give us a constant field along one axis,

$$
\begin{aligned}
\mathbf{B} & =\nabla \times \mathbf{A} \\
B_{z}=B_{3}=F_{12} & =\partial_{1} A_{2}-\partial_{2} A_{1} \\
& =\partial_{x} A_{y}-\partial_{y} A_{x} .
\end{aligned}
$$

There are multiple (in fact infinite) possible choices for $A_{\mu}(x)$ which lead to the same physical field $\mathbf{B}$. We commence with,

$$
A_{x}=-B y
$$

which gives a constant magnetic field of strength $B$ in the $z$-direction. We test that this gives the correct value for the magnetic field on the lattice by examining a single plaquette. A plaquette is the smallest example of a Wilson loop, which is a closed path of gauge links. It is calculated from the product of the gauge links over the path, with backwards links represented by Hermitian conjugates $U_{\mu}^{\dagger}(x)$. It was shown in Equation (3.8) that the field strength tensor is encoded in the plaquettes on the lattice,

$$
P_{\mu \nu}(x)=\exp \left(i q a^{2} F_{\mu \nu}(x)\right) .
$$

In our case of a constant background field this equation is exact because the higher order terms involve a derivative of at least second order. The definition of the background field link in Equation (4.3) is also exact in this case. 
For a general plaquette $P_{x y}$ (not near the boundary of the lattice) we get,

$$
e^{-i a e B y} e^{i a e B(y+a)}=e^{i a^{2} e B},
$$

as we hope. However, when we get to the boundary where $y=N_{y}-1$ there is a discontinuity in the gauge field due to the periodic boundary conditions which gives us,

$$
e^{-i a e B\left(N_{y} a-a\right)} e^{i a e B(0)}=e^{-i a^{2} e B\left(N_{y}-1\right)} .
$$

In order to correct for this discontinuity we use the only other free variable available in Equation (4.5), which is $A_{y}$. We give $A_{y}$ the following values,

$$
A_{y}=\left\{\begin{array}{cc}
N_{y} B x, & y / a=\left(N_{y}-1\right) \\
0, & \text { elsewhere }
\end{array}\right.
$$

Such that at the $y / a=N_{y}-1$ boundary we now get,

$$
\begin{aligned}
e^{-i a e B\left(N_{y} a-a\right)} e^{i a e B N_{y}(x+a)} e^{i a e B(0)} e^{-i a e B N_{y}(x)} & =e^{-i a e B\left(N_{y} a-a\right)} e^{i a^{2} e B N_{y}} \\
& =e^{i a^{2} e B} .
\end{aligned}
$$

as required. There is then another problem when we arrive at the double boundary, $x=N_{x}-1$ and $y=N_{y}-1$. At this point the result of the plaquette is,

$$
e^{-i a e B\left(N_{y} a-a\right)} e^{i a e B N_{y}(0)} e^{i a e B(0)} e^{-i a e B N_{y}\left(N_{x} a-a\right)}=e^{i a^{2} e B} e^{i a^{2} e B N_{y} N_{x}} .
$$

The only way for this to result in the value we want is if,

$$
e^{i a^{2} e B N_{y} N_{x}}=1
$$

which is only true under the condition [12],

$$
e B a^{2}=\frac{2 \pi n}{N_{x} N_{y}}
$$

Here $n$ can be any integer, so we have a quantisation condition which limits the available choices of magnetic field strength for a uniform field everywhere. This condition is very important in background field method calculations because the energy relation given in Eq. (4.1) is only valid for small fields. If we are working on a small lattice then the magnetic field will be large and higher order terms will begin to dominate. This motivates us to not only 

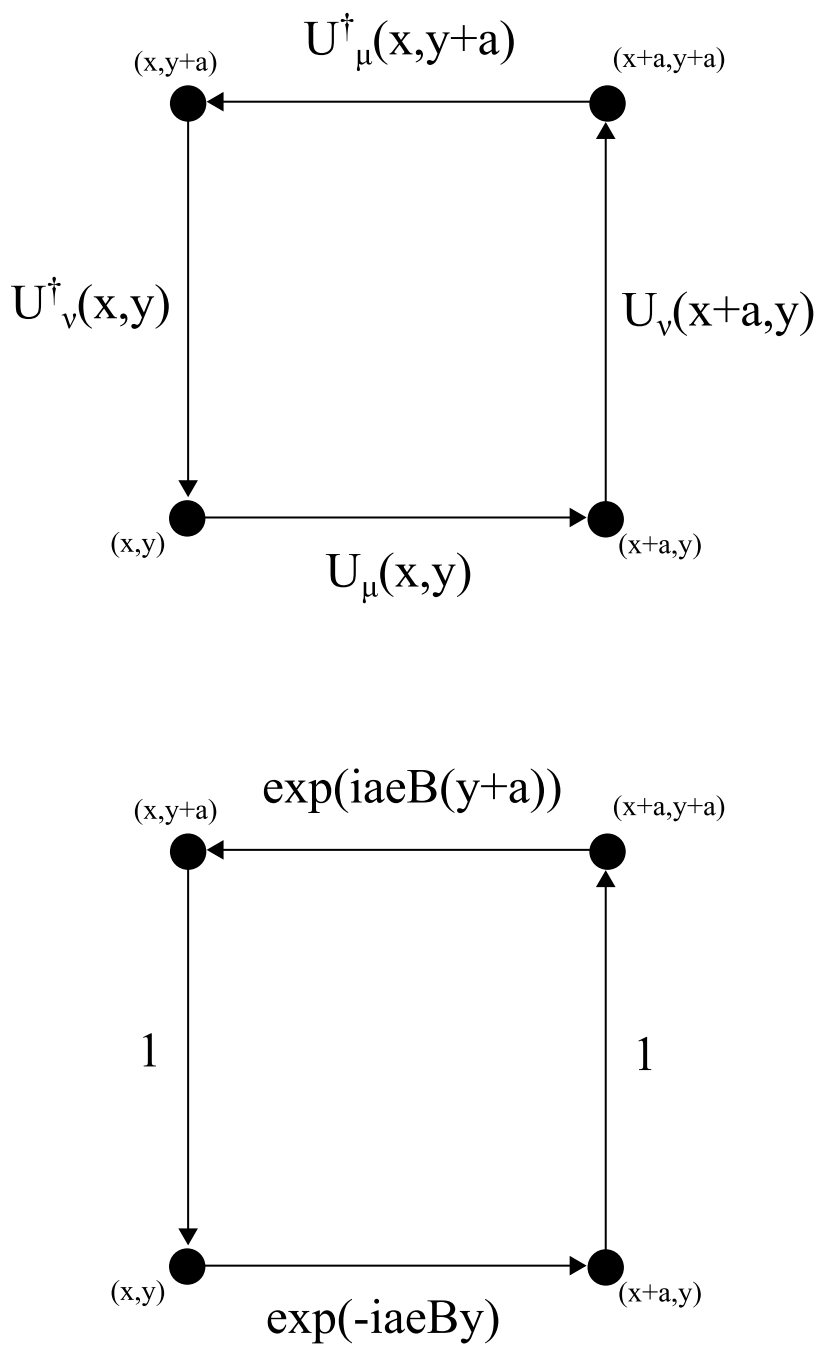

Figure 4.1: Top: The definition of a general plaquette of gauge links. Bottom: The values of the background field links $U_{\mu}^{(B)}(x)$ for a general plaquette away from the boundary. 

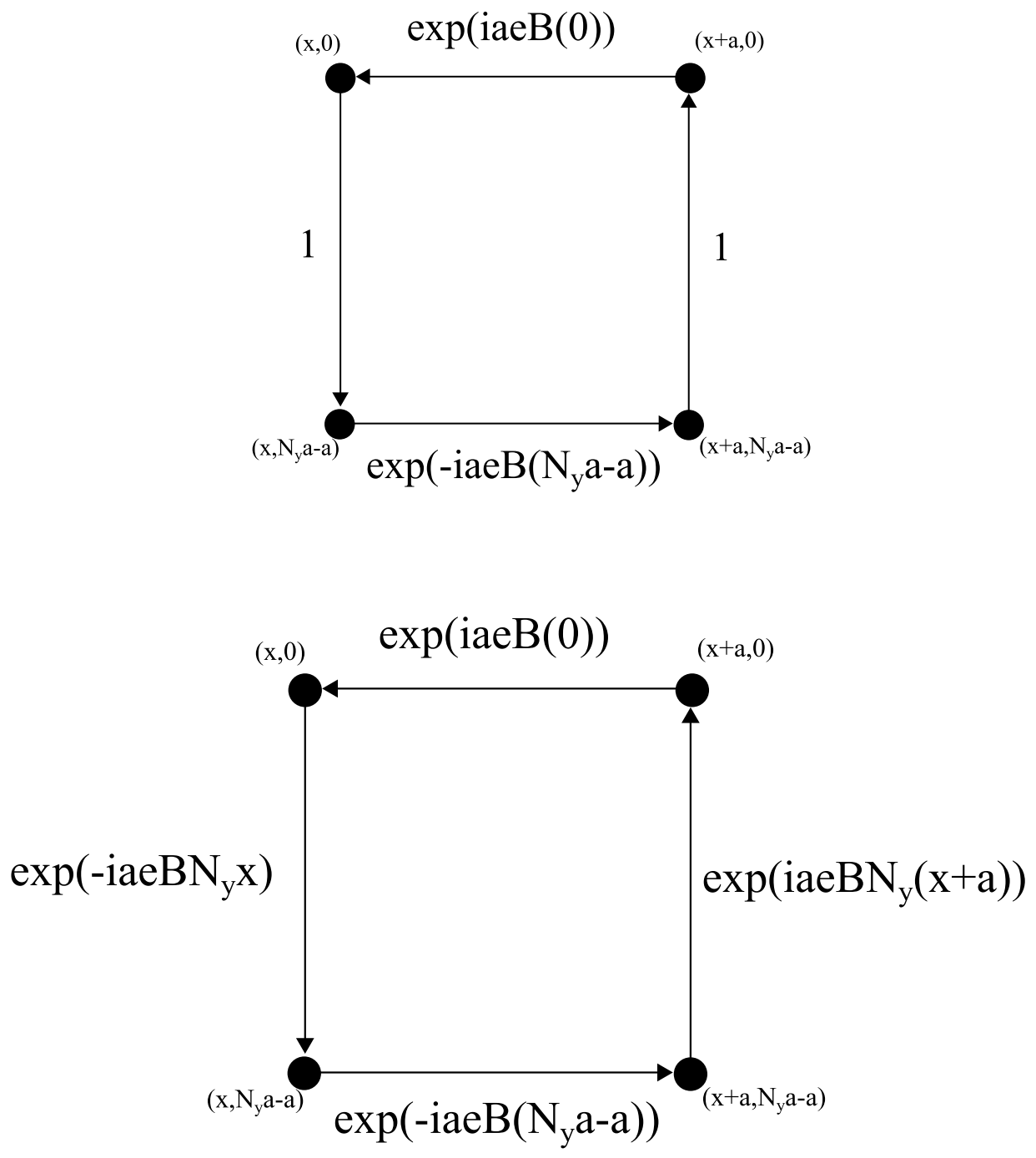

Figure 4.2: Top: A plaquette at the edge of the lattice, $y=N_{y}-1$. Bottom: The same plaquette with the boundary terms added to the background field links to correct for the discontinuity. 


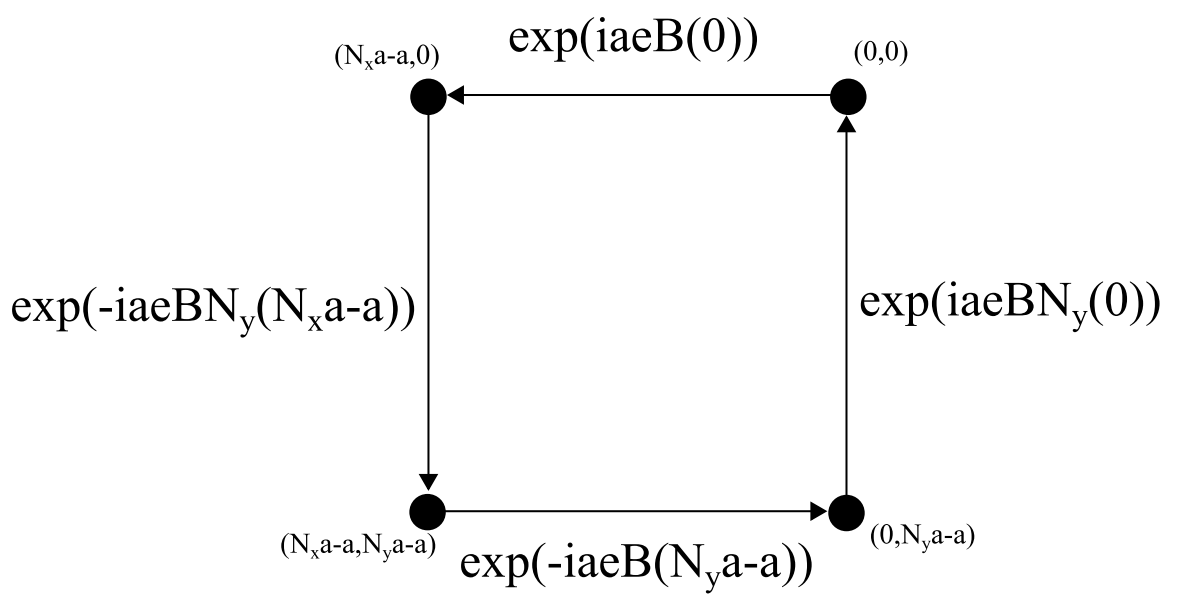

Figure 4.3: The plaquette at the corner of the lattice, $x=N_{x}-1, y=N_{y}-1$.

use a large number of lattice sites but also a relatively large lattice spacing a. However we still have to be careful of discretisation errors and our use of improved actions will be of utility.

Historically the computational cost for having a large enough lattice to meet the small field requirement has often been found to be impractical. As a result of this, other calculations have used ways of getting around the quantisation condition, allowing them to use smaller fields than the lattice would otherwise allow. One technique is to use a linearised version of the background field gauge links,

$$
U_{\mu}^{(B)}=e^{i a q A_{\mu}} \rightarrow 1+i a q A_{\mu},
$$

which has $\mathcal{O}\left(a^{2}\right)$ errors. It was shown in [20] that in the electric field case this introduces order $E^{2}$ errors and has a significant effect on the calculated polarisability. In our magnetic field case the errors would be order $B^{2}$ and we would expect a similarly significant impact on the magnetic polarisability results. One also has the difficulty of introducing a fixed boundary condition in a spatial direction of the lattice. Another technique is to use the full exponential form for the background field gauge links but avoid the quantisation condition by again using a fixed boundary condition in the $x$ direction [15]. An early paper [12] used the exponential phase factor and periodic boundary with the corrected phase along the boundary but ignored the quantisation 
condition from the corner. They put the quark source at the centre of the lattice to reduce the errors from the discontinuity in the magnetic field at the boundary.

Due to the unpredictability of the errors associated with these methods for avoiding the quantisation condition we are committed to using the fully quantised, uniform field for our calculations. 


\section{Chapter 5}

\section{Simulation Details}

Our background field method calculations primarily use the $2+1$ flavour dynamical QCD gauge configurations provided by the PACS-CS collaboration [22] through the International Lattice Data Grid [23]. They are $32^{3} \times 64$ lattices made using a non-perturbative $\mathcal{O}(a)$-improved Wilson quark action and Iwasaki gauge action. Simulations were carried out at $\beta=1.9$, corresponding to a lattice spacing in the physical limit of $a=0.0907(13) \mathrm{fm}$. We used four values of the hopping parameter $\kappa_{\mathrm{ud}}$ corresponding to pion masses from 622 to $282 \mathrm{MeV}$ with $\kappa_{\mathrm{s}}=0.13640$. The full details of each ensemble are given in Table 5.1, where $a$ determined from the Sommer parameter of $r_{0}=0.49 \mathrm{fm}$ are also provided.

\begin{tabular}{cccc}
$\kappa_{\text {ud }}$ & $m_{\pi}(\mathrm{MeV})$ & $\mathrm{a}(\mathrm{fm})$ & \# cfgs \\
\hline \hline 0.13700 & 622 & $0.1022(15)$ & 320 \\
0.13727 & 512 & $0.1009(15)$ & 320 \\
0.13754 & 388 & $0.0961(13)$ & 400 \\
0.13770 & 282 & $0.0951(13)$ & 400 \\
\hline
\end{tabular}

Table 5.1: Simulation details.

The Sommer scale [24] involves calculating the static quark potential and then fitting to the Sommer parameter, defined by,

$$
r_{0}^{2}=\left.\frac{d V(r)}{d r}\right|_{r=r_{0}}=1.65
$$

Given the physical value of the Sommer parameter $r_{0}=0.49 \mathrm{fm}$ one can then determine the lattice spacing. 
It should be noted that these configurations are dynamical only in the QCD sense. There was no magnetic field included when they were calculated, so the sea quarks do not "see" the field. The background field can be put on the sea quarks straightforwardly by generating a separate Markov chain for each field strength using the background field modified gauge links in the fermion action. This is obviously very computationally expensive because the calculation of the fermion determinant is already the most difficult part of the gauge field generation. The other problem with this approach is that because there is a separate ensemble for each field strength the correlation between the zero and non-zero field correlation functions is lost. Our results exploit this correlation extensively. Without this, tens of thousands of dynamical gauge fields are required and must be left for future consideration.

Alternatively to the full calculation there exist techniques for a reweighting of configurations in order to correct for the background field [25]. This is less computationally intensive than just recalculating each gauge field, but the extent to which the necessary correlations are preserved remains to be investigated. In this work we ignore the effect of the background field on the sea quarks. While we expect these contributions to be small, it will be important to eventually include them in future precision comparisons with experiment.

For our propagators we use a Clover fermion action with $C_{S W}=1.715$ determined non-perturbatively [8]. Unless otherwise specified, all our propagators use the interpolating fields,

$$
\chi_{1}^{p}=\left(u^{T} C \gamma_{5} d\right) u
$$

for the proton and for neutron,

$$
\chi_{1}^{n}=\left(d^{T} C \gamma_{5} u\right) d .
$$

In order to improve ground state overlap we employ fermion source smearing on our interpolating fields using stout-smeared links. Again unless otherwise specified, all propagators used 100 sweeps of gauge-invariant Gaussian smearing at the source with a smearing coefficient of 0.7. We used periodic boundary conditions in all the spatial dimensions and a fixed boundary condition in time.

We calculated propagators at six non-zero field strengths, corresponding to $n=1,-2,3,4,-6,-8$ in the quantisation condition given by Eq. (4.13). Using the relationships $q_{d}=-e / 3$ and $q_{u}=2 e / 3$ to combine up and down 
quark propagators with the appropriate field strengths resulted in hadrons in fields of strength $e B=-0.087,+0.174,-0.261,-0.345 \mathrm{GeV}$ at the physical lattice spacing. This is not quite as efficient as the choice of fields we used in our quenched calculation (Appendix A.1), but gives us all of the four smallest available field strengths.

\subsection{Error analysis}

The standard deviation for a large sample of uncorrelated measurements can be written as,

$$
\sigma_{x}=\sqrt{\frac{1}{N} \sum_{i=1}^{N}\left(x_{i}-\bar{x}\right)^{2}},
$$

where $N$ is the sample size, $x_{i}$ is the value for measurement $i$ and $\bar{x}$ is the sample average. In using Eq. (5.4) each entry of $x_{i}$ must be a measurement. However, in Monte Carlo, each sample need not be a measurement. Only after averaging over several samples does one obtain an estimate suitable for Eq. 5.4. We therefore perform our error analysis using the jacknife method. Performing a jackknife involves creating $N$ subensembles by removing the $n$th configuration of the original ensemble,

$$
X_{n}=\frac{1}{N-1} \sum_{i=1, i \neq n}^{N} x_{i} .
$$

The variance is then given by,

$$
\sigma_{x}^{2} \equiv \frac{N-1}{N} \sum_{i=1}^{N}\left(X_{i}-\bar{X}\right)^{2}
$$

where $\bar{X}$ is the mean of all the $X_{i}$ subensemble measurements. In order to get errors for the correlation functions, the energy shifts, and the moment/polarisability values we use a third order jacknife. This requires a complement set with the $i$ th, $j$ th, and $k$ th configurations removed for all values of $i, j$, and $k$. One challenge of a third order jackknife are the huge

memory requirements, which scale approximately like $\mathcal{O}\left(N^{3}\right)$, such that we sometimes have to group some configurations into bins. 
Our fits of the effective energies and of the energy shift as a function of the background field were performed using linear least squares fits. The naive $\chi^{2}$ is defined by,

$$
\chi^{2}=\sum_{i=1}^{k}\left(\frac{X_{i}-\mu_{i}}{\sigma_{i}}\right)^{2},
$$

where $k$ is the number of points, $X_{i}$ is the value at point $i, \mu_{i}$ is the value predicted by the fit at that point, and $\sigma_{i}$ is the error at that point. The degrees of freedom is just the number of data points minus the number of fit parameters ( 1 for effective mass fits). The $\chi_{\text {dof }}^{2}$ is a measure of how well the data fits the model, with a value on the order of 1 suggesting a good fit. However, there can again be correlation in the values used in the fits, such as between adjacent time slices when fitting the effective mass or between background field strengths when fitting $\mu$ or $\beta$. This would lead to overconfidence in the quality of our fits, so we take this into account by using the full covariance matrix in the calculation of our $\chi_{\text {dof }}^{2}$ values,

$$
\chi^{2}=\sum_{i, j=1}^{k}\left(X_{i}-\mu_{i}\right)\left(C^{-1}\right)_{i j}\left(X_{j}-\mu_{j}\right),
$$

where $C$ is the covariance matrix, defined by,

$$
C_{i j}=(N-1)\left(\overline{X_{i} X_{j}}-\bar{X}_{i} \bar{X}_{j}\right),
$$

where $\bar{X}_{i}$ and $\bar{X}_{j}$ are the ensemble averages at points $i$ and $j$ and $\overline{X_{i} X_{j}}$ is the ensemble average of the product of the two points. This gives an accurate measure of the quality of the fit. 


\section{Chapter 6}

\section{Landau Levels}

\subsection{Introduction}

Observe the equation which describes the energy for a particle in a uniform magnetic field $[9,11]$,

$$
E(B)=M-\boldsymbol{\mu} \cdot \mathbf{B}+\frac{e|B|}{2 M}-\frac{4 \pi}{2} \beta B^{2}+\mathcal{O}\left(B^{3}\right) .
$$

In addition to the bare mass and the terms involving the magnetic moment and polarisability, there is another term which is proportional to the magnitude of the magnetic field,

$$
\frac{e|B|}{2 M}
$$

where $e>0$ is the elementary charge, $B$ is the magnetic field strength and $M$ is the mass of the particle. This term is called the Landau energy, and it arises from the quantisation of angular momentum for a charged particle in a magnetic field. The charge in this case refers to the overall charge of the hadron, so it is expected to be zero for a neutral particle such as the neutron. For the proton however the term is non-zero, and has a significant effect when performing background field calculations.

When calculating magnetic moments the relevant term in the energy relation of Eq. (6.1) is isolated from the other terms by taking a difference of correlation functions. Taking the difference of a spin-up and spin-down effective mass or taking the difference of positive and negative fields causes all the terms in the equation except for the magnetic moment term to cancel, including the Landau energy term, leaving terms of order $B^{3}$. This allows us to 
calculate magnetic moments of charged particles without direct interference by the Landau energy term.

The magnetic polarisability term is isolated by taking the average of spinup and spin-down or positive and negative fields to remove the moment term, and then subtracting off the zero-field mass separately. This however does not remove the Landau energy term, which cannot be cancelled by any combination of spins or fields. This means that the effective energies that we must try to fit in order to calculate magnetic polarisabilities will always include this extra energy for charged particles. It is possible to calculate analytically the size of the Landau energy given we know the charge and mass of the particle and the strength of the magnetic field. This value could then be subtracted from the spin averaged effective mass to leave only the contribution from the polarisability. In practice however, this won't necessarily work because Eq. (6.1) only tells part of the story. The energy-field relation describes the shift in energy due to a magnetic field for the ground state of a particle. In addition to the ground state Landau energy there exists an infinite tower of Landau levels with energy given by,

$$
E_{n}=\frac{e|B|}{2 M}(2 n+1) \text { for } n=0,1,2,3, \ldots
$$

All of these levels contribute to the background field correlation function. Like any excited state these are exponentially suppressed in large Euclidean time. Unfortunately at typical field strengths used in background field calculations the difference between the energy levels is much less than the difference between, say, the nucleon and its first excited state (the Roper). This means that it takes much longer for the higher Landau levels to be suppressed and leave only the ground level, by which time it is likely that the signal will be overcome by noise.

Another problem created by the Landau levels relates to momentum states. The Landau energy comes from charged hadrons with non-zero orbital angular momentum. The typical effective mass calculation used in background field calculations involves projection to a zero momentum plane wave eigenstate with coordinate-space wavefunction,

$$
\psi_{\mathbf{p}}=e^{i \mathbf{p} \cdot \mathbf{x}},
$$

and thus will project a superposition of Landau levels. This will affect the proton magnetic moment results as one relies on the cancellation of the energy associated with the Landau levels in subtracting different spin or field 
orientations. We have found that the effect of this on the magnetic moment calculation appears to be small as the proton effective energies used to calculate the magnetic moment are similar in quality to those for the neutron. This can be seen in the effective energy plots in Section 8. In the future it would be interesting to project to the lowest Landau level. In this investigation we will probe the nature of this state in a uniform field.

The way the Landau levels could be handled is to replace the projection to a plane wave with a projection to the lowest Landau level.

$$
G(t, \mathbf{B})=\sum_{\mathbf{x}} \psi_{0}^{L}(x)\langle 0|\chi(x) \bar{\chi}(0)| 0\rangle
$$

which results in a correlation function where only the lowest Landau level contributes to the energy of each state. In Ref. [21] this technique was investigated using the wave function of a ground-state harmonic oscillator,

$$
\psi_{0}^{L}(x)=e^{-\frac{1}{2} e|B| x^{2}},
$$

They investigated the finite volume and finite lattice spacing effects on the Landau level wavefunction and found that the discretisation effects on the wave function of the lowest Landau level were negligible, but that the finite volume effects were important, motivating the determination of the finite volume Landau ground state wave function for projection. We hope to perform further research into this technique in the future.

\subsection{Derivation}

The Landau levels can be derived [26] by considering the Dirac equation for a point-like particle with charge $e$ in an external electromagnetic field defined by its potential $A_{\mu}(x)$,

$$
\left(i \gamma^{\mu} \partial_{\mu}-e \gamma^{\mu} A_{\mu}-m\right) \psi(x)=0
$$

This can be rewritten more explicitly by breaking $\gamma^{\mu}$ into $\gamma^{0}$ and $\gamma^{i}$ and then multiplying both sides by $\gamma^{0}$,

$$
\gamma^{0} i \gamma^{0} \frac{\partial \psi}{\partial t}=\gamma^{0}\left(\gamma^{i} \hat{p}_{i}+e \gamma^{i} A_{i}+e \gamma^{0} A_{0}+m\right) \psi
$$


We then choose the Dirac representation of the gamma matrices and write this as,

$$
i \frac{\partial \psi}{\partial t}=\left(\boldsymbol{\alpha} \cdot \hat{\mathbf{p}}-e \boldsymbol{\alpha} \cdot \mathbf{A}+e A^{0}+\beta m\right) \psi
$$

where the matrices are defined,

$$
\beta\left(=\gamma^{0}\right)=\left(\begin{array}{cc}
I & 0 \\
0 & -I
\end{array}\right), \quad \boldsymbol{\alpha}\left(=\gamma^{0} \gamma^{i}\right)=\left(\begin{array}{cc}
0 & \boldsymbol{\sigma} \\
\boldsymbol{\sigma} & 0
\end{array}\right) .
$$

One then writes $\psi=\left(\begin{array}{l}\phi \\ \chi\end{array}\right)$, which leads to the pair of coupled equations

$$
\begin{aligned}
i \frac{\partial \phi}{\partial t} & =\boldsymbol{\sigma} \cdot(\hat{\mathbf{p}}-e \mathbf{A}) \chi+e A^{0} \phi+m \phi \\
i \frac{\partial \chi}{\partial t} & =\boldsymbol{\sigma} \cdot(\hat{\mathbf{p}}-e \mathbf{A}) \phi+e A^{0} \chi-m \chi
\end{aligned}
$$

Then, as with the background field method, we choose a vector potential that gives a constant magnetic field $\mathbf{B}$ along the $z$ axis, $A^{0}=A^{x}=A^{z}=0$, $A^{y}=B x$. We take a stationary solution of energy $E, \psi=e^{-i E t}\left(\begin{array}{l}\phi \\ \chi\end{array}\right)$, so that Eq. (6.11) and (6.12) become,

$$
\begin{aligned}
(E-m) \phi & =\boldsymbol{\sigma} \cdot(\hat{\mathbf{p}}-e \mathbf{A}) \chi \\
(E+m) \chi & =\boldsymbol{\sigma} \cdot(\hat{\mathbf{p}}-e \mathbf{A}) \phi
\end{aligned}
$$

which we can combine to eliminate $\chi$ and get an equation for $\phi$ :

$$
\begin{aligned}
\left(E^{2}-m^{2}\right) \phi & =[\boldsymbol{\sigma} \cdot(\hat{\mathbf{p}}-e \mathbf{A})]^{2} \phi \\
& =\left[(\hat{\mathbf{p}}-e \mathbf{A})^{2}-e \boldsymbol{\sigma} \cdot \mathbf{B}\right] \phi \\
& =\left[\hat{\mathbf{p}}^{2}+e^{2} B^{2} x^{2}-e B\left(\sigma_{z}+2 x p_{y}\right)\right] \phi \\
& =\left[\hat{\mathbf{p}}^{2}+\left(e B x-p_{y}\right)^{2}+e B \sigma_{z}\right] \phi .
\end{aligned}
$$

This is the Hamiltonian of a harmonic oscillator. Since $p_{y}, p_{z}$ and $\sigma_{z}$ commute with the right-hand side, we constrain the particle to the $x-y$ plane (by setting $p_{z}=0$ ) for simplicity and look for a solution of the form

$$
\phi(x)=e^{i\left(p_{y} y\right)} f(x)
$$


where $f(x)$ satisfies equation (6.15),

$$
\left[-\frac{d^{2}}{d x^{2}}+\left(e B x-p_{y}\right)^{2}-e B \sigma_{z}\right] f(x)=\left(E^{2}-m^{2}\right) f(x) .
$$

Constraining $B$ to be positive such that $e B>0$, we define extra variables,

$$
\begin{aligned}
\omega & =\sqrt{e B}\left(x-\frac{p_{y}}{e B}\right) \\
a & =\frac{E^{2}-m^{2}}{e B},
\end{aligned}
$$

so that Eq. (6.17) simplifies to,

$$
\left(-\frac{d^{2}}{d \omega^{2}}+\omega^{2}-\sigma_{z}\right) f=a f
$$

If $f$ is an eigenvector of $\sigma_{z}$ with eigenvalue $\alpha= \pm 1$,

$$
\begin{aligned}
& f=\left(\begin{array}{c}
f_{1} \\
0
\end{array}\right) \text { for } \alpha=1 \\
& f=\left(\begin{array}{c}
0 \\
f_{-1}
\end{array}\right) \text { for } \alpha=-1
\end{aligned}
$$

then $f_{\alpha}$ satisfies

$$
\left(\frac{d^{2}}{d \omega^{2}}-\omega^{2}\right) f_{\alpha}(\omega)=-(a+\alpha) f_{\alpha}(\omega), \quad \alpha= \pm 1
$$

which has the solution, written in terms of a Hermite polynomial $H_{n}(\omega)$ :

$$
f_{\alpha}=c e^{-\omega^{2} / 2} H_{n}(\omega)
$$

Given that $H_{0}=1$, one can see that this has the same form as the suggested ground state wavefunction in Eq. 6.6. However this is only a solution if we have $a+\alpha=2 n+1$, for $n=0,1,2, \ldots$ Therefore the energy levels are given by,

$$
\begin{aligned}
E^{2} & =m^{2}+e B(2 n+1-\alpha) \\
E & =m \sqrt{1+\frac{e B}{m^{2}}(2 n+1-\alpha)}
\end{aligned}
$$


Then using the small $x$ expansion of $\sqrt{1+x}$ with $x=\frac{e B}{m^{2}}(2 n+1-\alpha)$,

$$
\sqrt{1+x}=1+\frac{x}{2}-\frac{x^{2}}{8}+\frac{x^{3}}{16}+\mathcal{O}\left(x^{4}\right)
$$

we can get the required energy expansion:

$$
\begin{aligned}
E= & m+\frac{e B}{2 m}(2 n+1)-\alpha \frac{e B}{2 m}-\frac{(e B)^{2}}{8 m^{3}}(2 n+1-\alpha)^{2} \\
& +\frac{(e B)^{3}}{8 m^{5}}(2 n+1-\alpha)^{3}+\mathcal{O}\left(\frac{(e B)^{4}}{m^{7}}\right) .
\end{aligned}
$$

The first term is just the mass and the second term describes the energy due to the Landau levels. The third term is actually equivalent to the magnetic moment term in Eq. (6.1), as it is proportional to $\alpha$, which is the eigenvalue of $\sigma_{z}$ which appears in the spin operator. The higher order terms can safely be ignored for $e B \ll m$. Therefore we have shown that the Landau levels for a charged particle in a magnetic field are given by,

$$
E_{\text {Landau }}=\frac{e|B|}{2 m}(2 n+1) \text { for } n=0,1,2,3, \ldots
$$

with a wavefunction proportional to Eq. 6.6 for a particle of charge $e$ and mass $m$ in a field $B$.

\subsection{Examples}

We can see evidence of the Landau levels in some of our energy shift plots. These are plots of the average of spin-up and spin-down energies with the zero-field mass subtracted off. That means that the only effects that should be present are the magnetic polarisability, the Landau energy and some negligible higher order contributions.

Figure 6.1 displays an effective energy plot for the average of spin-up and spin-down protons in a magnetic field with the zero-field mass subtracted. This is from our earlier (quenched) calculation using the heaviest quark mass considered at the smallest background field strength. The details of this calculation are found in Appendix A. We notice that there were two very distinct plateaus that occur at different energy levels with a constant downward slope between them. We fit these plateaus and find values of $45.3 \mathrm{MeV}$ and $19.5 \mathrm{MeV}$, a difference of approximately $26 \mathrm{MeV}$. Using knowledge of 


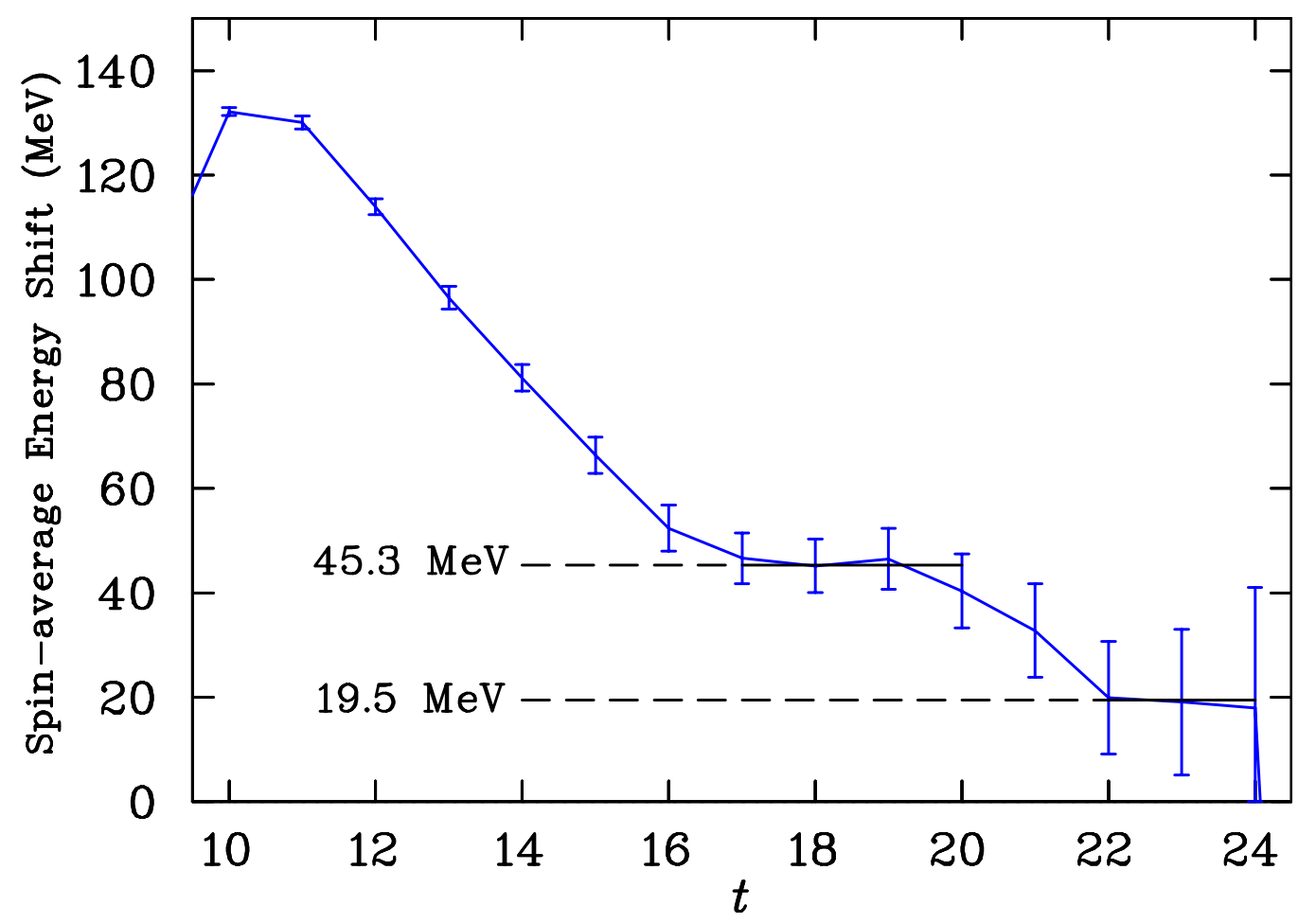

Figure 6.1: Spin-averaged energy shift from an earlier quenched background field calculation. 
the lattice parameters and the magnetic field that we are working with, we can calculate the expected difference in energies between two Landau levels. As derived in Eq. (6.19) the difference between the first and second Landau levels (or any adjacent levels) is given by,

$$
\frac{e|B|}{2 M}(3-1)=\frac{e|B|}{M}
$$

where $e$ is the charge of the particle, $B$ is the strength of the magnetic field and $m$ is the mass of the particle.

Our quenched calculation used a $32^{3}$ spatial lattice with lattice spacing $0.127 \mathrm{fm}$. For the mass we have to use the mass calculated on this lattice, rather than the physical mass, so $M=1670 \mathrm{MeV}$.

$$
\begin{aligned}
\frac{e|B|}{M} & =\frac{3 \cdot 2 \pi}{n_{x} n_{y} a^{2}} \frac{1}{M} \\
& =\frac{3 \cdot 2 \pi}{32^{2}(0.127 \mathrm{fm})^{2}} \frac{1}{1670 \mathrm{MeV}} \\
& =26 \mathrm{MeV},
\end{aligned}
$$

where the factor of 3 is associated with the quark charge. This is almost exactly what is given by the difference in plateaus from the effective energy plot. We are therefore led to believe that the drop that we see from one energy level to another is due to the Landau levels. The experimental value for the magnetic polarisability of the proton is positive and the polarisability term in Eq. (6.1) has a minus sign. This means that the contribution to the effective energy from the polarisability should be negative. The fact that we see the energy shift as positive in this region is thus due to the Landau levels.

We can also calculate the expected energy shift due to the polarisability using the experimental value, which for the proton is $1.9 \pm 0.5\left(10^{-4} \mathrm{fm}^{3}\right)[27]$,

$$
\begin{aligned}
\frac{4 \pi}{2} \beta B^{2} & =\frac{4 \pi}{2} \beta \frac{\left(e B a^{2}\right)^{2}}{e^{2} a^{4}} \\
& =\frac{4 \pi}{e^{2}} \frac{\left(e B a^{2}\right)^{2}}{2 a^{4}} \beta \\
& =\frac{137}{2(0.127 \mathrm{fm})^{4}} \frac{3 \cdot 2 \pi}{32^{2}}\left(1.9 \times 10^{-4} \mathrm{fm}^{3}\right) \\
& =3.3 \mathrm{MeV}
\end{aligned}
$$

This value is very small, only a fraction of even the lowest Landau level energy, due to the fact that the lattice spacing is quite coarse, allowing a 


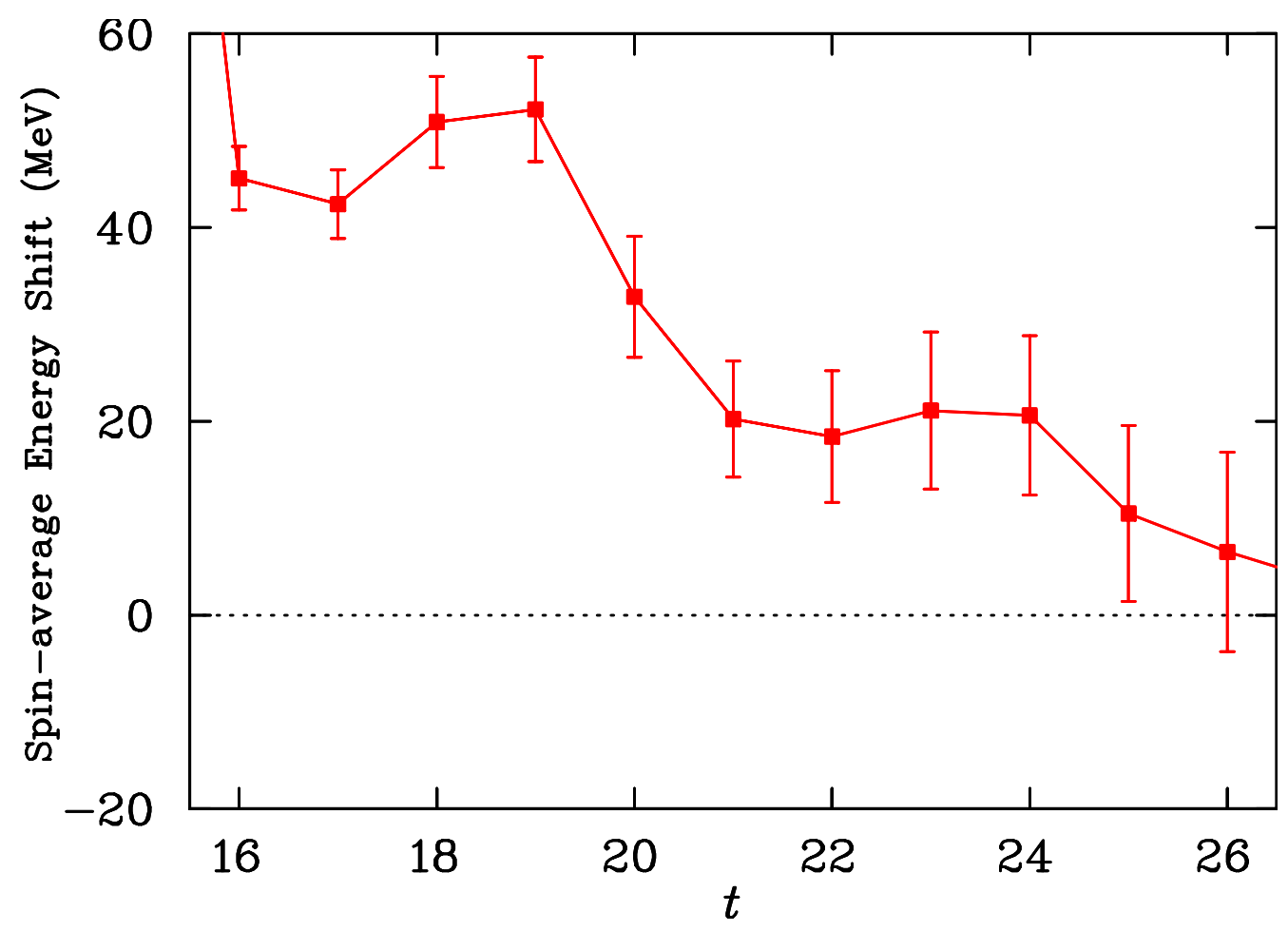

Figure 6.2: Spin-averaged energy shift from our dynamical background field calculation.

small field. Since it is so small it may not be possible to extract this shift even if we project to the lowest Landau level. In the example from Fig. 6.1 if we assume that the levels we have fit are the third and second Landau levels then subtracting the energy of the lowest level from the second plateau results in a value which is consistent with a shift of $3 \mathrm{MeV}$. This relies on the fact that by this time the errors have grown quite large, and the value is also consistent with zero. Therefore it may be necessary to use somewhat larger background fields for calculating polarisabilities of charged particles simply because the shift will otherwise be too small to resolve.

Figure 6.2 displays another effective energy plot for the average of spin-up and spin-down in a magnetic field with the zero-field mass subtracted, this time from our dynamical calculation. Again it is at the heaviest available quark mass (although much lighter than the heaviest quenched calculation) and the smallest magnetic field. Here we again see multiple plateaus, although not nearly as clearly as in the quenched case. There is a sort of 
plateau at 18-19 and then a quick drop to a quite clear plateau at 21-24. This is followed by a downwards drift to zero at 25-28 which could be fit as a plateau due to the large errors at those times. We can again calculate the expected Landau levels given the properties of this lattice, with lattice spacing $a=0.10224 \mathrm{fm}$ and lattice nucleon mass $M=1420 \mathrm{MeV}$,

$$
\begin{aligned}
\frac{e|B|}{M} & =\frac{3 \cdot 2 \pi}{n_{x} n_{y} a^{2}} \frac{1}{M} \\
& =\frac{3 \cdot 2 \pi}{32^{2}(0.10224 \mathrm{fm})^{2}} \frac{1}{1420 \mathrm{MeV}} \\
& =48 \mathrm{MeV}
\end{aligned}
$$

This is larger than for the quenched case due to the finer lattice spacing and smaller nucleon mass. Therefore we would expect the Landau levels to be suppressed more quickly. Comparing this value to the plateaus in the effective energy plot we see that it is too large to be associated with the plateau in the middle at about $20 \mathrm{MeV}$. The value is consistent with the difference between the first plateau at time slice 18 and the last time slice before signal is lost, where the value is approximately zero. We also see that the value is again positive up until the loss of signal, in conflict with the negative mass shift we expect from the polarisability.

We can again calculate the expected energy shift due to the effect of the polarisability,

$$
\begin{aligned}
\frac{4 \pi}{2} \beta B^{2} & =\frac{4 \pi}{2} \beta \frac{\left(e B a^{2}\right)^{2}}{e^{2} a^{4}} \\
& =\frac{4 \pi}{e^{2}} \frac{\left(e B a^{2}\right)^{2}}{2 a^{4}} \\
& =\frac{137}{2(0.10224 \mathrm{fm})^{4}} \frac{3 \cdot 2 \pi}{32^{2}}\left(1.9 \times 10^{-4} \mathrm{fm}^{3}\right) \\
& =8.0 \mathrm{MeV} .
\end{aligned}
$$

The only thing which has changed from the quenched calculation is that the lattice is finer, which leads to a larger field strength. This value is larger than the one from the quenched lattices, but is still small compared to the Landau level energies. Due to the large errors and inconclusive plateaus it is probably not worth trying to draw too many conclusions about which levels we might be seeing. 
These examples show clearly that the Landau levels are affecting background field method effective energy plots. It is also clear that the lowest level is not the only one which is present. We believe that implementing a projection to the finite volume wave function of the lowest Landau level will allow us to remove the contributions coming from the higher levels. From there we hope that we can simply calculate the Landau contribution and subtract it in order to get the pure polarisability based mass shift. If the technique works it may be that the error associated with the Landau energy makes it difficult to resolve a value when the field is weak and therefore the shift is small. Since the Landau levels scale with $B$ and the polarisability term scales with $B^{2}$ there could arise problems when going to very small field strengths, making it harder to achieve the same level of precision we could get with neutral particle polarisabilities. These are interesting issues to be investigated in the future. 


\section{Chapter 7}

\section{Twisted Boundary Conditions}

\subsection{Introduction}

Twisted boundary conditions (TBCs) are a technique used in lattice QCD to access momenta other than the usual multiple of $2 \pi / L$ on a lattice with spatial volume $L^{3}[28,29]$. A phase or "twist" of $e^{i \phi}$ is introduced to the gauge field, which shifts the momentum of a particle by $\phi / L$ in the direction altered by the phase. The phase can be introduced in a number of ways, such as in a chunk on the boundary, or uniformly through the lattice with phase $e^{i \phi / L}$. Although twisted boundary conditions are not directly related to the background field method, they are important to understand due to the way they can arise naturally in background field calculations.

The boundary phase $\phi$ can be spread out across all the sites of the lattice via gauge transformation. This looks similar to the background field method, which also introduces a phase at all sites of the lattice, except that in twisted boundary conditions the phase is constant. Twisted boundary conditions in background field calculations were touched on briefly in Ref. [30] in the context of electric polarisabilities.

In the infinite volume limit a translation of a component of the gauge field such as,

$$
A_{x}=B y \rightarrow B\left(y-y_{0}\right),
$$

is a simple gauge transformation and therefore has no effect on any calculated

observables. If we define the origin of the gauge field as the point where $\vec{A}=\overrightarrow{0}$, then this translation moves the origin of the field from $y=0$ to $y=y_{0}$. 
When formulating the background field method in Section 4 we chose $A_{x}=-B y$ in order to produce a background field $B$. Had we chosen $A_{x}=$ $-B\left(y-y_{0}\right)$ the resultant background field would have been the same. Due to the periodic boundary conditions on the lattice, however, these choices are not directly related by a gauge transformation. Making the second choice for $A_{x}$ results in the following change in the gauge links,

$$
U_{1}^{(B)}(x)=e^{i \omega y} U_{1}(x) \rightarrow U^{\prime(B)}(x)=e^{i \omega\left(y-y_{0}\right)} U_{1}(x),
$$

where $U_{1}(x)$ is the pure QCD gauge link. As stated this does not change the magnetic field induced on the lattice and therefore does not affect the energy shift due to the magnetic moment or polarisability. However it does change the nature of the two-point function calculated on this transformed field. This will be explored in detail.

We can rewrite the modified links as,

$$
e^{i \omega\left(y-y_{0}\right)} U_{1}(x)=e^{i \omega y_{0}} e^{i \omega y} U_{1}(x)
$$

so we can see that we have effectively multiplied each $x$-direction gauge link on the lattice by a constant phase $e^{i \omega y_{0}}$. This phase appears to be equivalent to a twisted boundary conditions phase.

In this chapter we will first briefly describe how twisted boundary conditions work. We will use tree level lattice calculations to demonstrate the effect of twisted boundary conditions on an effective mass. However, we will then show that when combined with a quantised background magnetic field a twist-like phase has no effect on the physical states. Finally we will explain why this happens by illustrating how the phase can be removed through a gauge transformation making use of the periodic boundary conditions and the quantised form of the background field. 


\subsection{Twisted boundary conditions and momentum shifts}

Begin by considering the naive fermion action on the lattice, with two degenerate flavours of quarks and gauge links $U_{\mu}(\mathbf{x})$ modified by a $U(1)$ phase $\exp \left(i a A_{\mu}(\mathbf{x})\right)$

$$
\begin{aligned}
S_{q}=\frac{1}{2 a} \sum_{\mathbf{x}, \hat{\mu}} \bar{q}(\mathbf{x}) \gamma^{\mu}\left[U_{\mu}(\mathbf{x}) \exp \left(i a A_{\mu}(\mathbf{x})\right) q(\mathbf{x}+\hat{\mu})-\right. \\
\left.\quad U_{\mu}^{\dagger}(\mathbf{x}-\hat{\mu}) \exp \left(-i a A_{\mu}(\mathbf{x}-\hat{\mu})\right) q(\mathbf{x}-\hat{\mu})\right]+m_{q} \sum_{\mathbf{x}, \hat{\mu}} \bar{q}(\mathbf{x}) q(\mathbf{x})
\end{aligned}
$$

Here we have quark fields $q(x)$ and $\bar{q}(x)$ which satisfy periodic boundary conditions and we take the $U(1)$ field to have the value

$$
\mathbf{A}=\left(0,0,0, \frac{\omega}{L}\right)=\frac{\omega}{L} \hat{z}
$$

The constant field can be described by splitting the term into two z-dependent parts that act on the quark fields

$$
\begin{array}{r}
S_{q}=\frac{1}{2 a} \sum_{\mathbf{x}, \hat{\mu}} \exp (-i a(\omega / L) \mathbf{z}) \bar{q}(\mathbf{x}) \gamma^{\mu}\left[U_{\mu}(\mathbf{x}) \exp (i a(\omega / L)(\mathbf{z}+\hat{z})) q(\mathbf{x}+\hat{\mu})-\right. \\
\left.U_{\mu}^{\dagger}(\mathbf{x}-\hat{\mu}) \exp (i a(\omega / L)(\mathbf{z}-\hat{z})) q(\mathbf{x}-\hat{\mu})\right]+m_{q} \sum_{\mathbf{x}, \hat{\mu}} \bar{q}(\mathbf{x}) q(\mathbf{x}) .
\end{array}
$$

Here we have defined $\mathbf{z}=z \hat{z}=(0,0,0, z)$. We can then choose to redefine our quark field variables to absorb the terms that pre-multiply them.

$$
\begin{aligned}
& \tilde{q}(\mathbf{x})=\exp (i a(\omega / L) \mathbf{z}) q(\mathbf{x}) \\
& \tilde{q}(\mathbf{x})=\exp (-i a(\omega / L) \mathbf{z}) \bar{q}(\mathbf{x})
\end{aligned}
$$

Such that our fermion action is now given by

$\tilde{S}_{q}=\frac{1}{2 a} \sum_{\mathbf{x}, \hat{\mu}} \tilde{\bar{q}}(\mathbf{x}) \gamma^{\mu}\left[U_{\mu}(\mathbf{x}) \tilde{q}(\mathbf{x}+\hat{\mu})-U_{\mu}^{\dagger}(\mathbf{x}-\hat{\mu}) \tilde{q}(\mathbf{x}-\hat{\mu})\right]+m_{q} \sum_{\mathbf{x}, \hat{\mu}} \tilde{\bar{q}}(\mathbf{x}) \tilde{q}(\mathbf{x})$, 
which is the same as Eq. (7.4) except that the $U(1)$ field is gone and the quark fields now satisfy twisted boundary conditions in the $z$ direction instead of periodic ones,

$$
\begin{aligned}
& \tilde{q}(z+L)=e^{i \omega} \tilde{q}(z) \\
& \tilde{\bar{q}}(z+L)=e^{-i \omega} \tilde{\bar{q}}(z) .
\end{aligned}
$$

We can then write the resultant free propagators in terms of both $q(\mathbf{x})$ and $\tilde{q}(\mathbf{x})$

$$
\tilde{S}(\mathbf{x}) \equiv\langle\tilde{q}(\mathbf{x}) \overline{\tilde{q}}(\mathbf{x})\rangle=\int \frac{d k_{4}}{2 \pi} \frac{1}{L^{3}} \sum_{\mathbf{k}} \frac{e^{i(k+\omega \hat{z} / L) \cdot \mathbf{x}}}{i \gamma^{\mu}\left(k_{\mu}+\frac{\omega \hat{z}}{L}\right)+M}=e^{i(\omega \hat{z} / L) \cdot \mathbf{x}} S(\mathbf{x}),
$$

where the sum is over lattice momenta $\mathbf{k}=(2 \pi / L) \mathbf{n}$ and $\mathbf{n}$ is a vector of integers. $\tilde{S}(\mathbf{x})$ satisfies twisted boundary conditions and $S(\mathbf{x})$ satisfies periodic boundary conditions. The momentum in the denominator of the propagator has been shifted by $\omega / L$ in the $z$-direction, such that there is now no zero momentum state.

\subsubsection{Examples}

In order to examine the effects of twisted boundary conditions we make use of tree level calculations. We do this because the effect of twisted boundary conditions when using a small twist can be quite small and when using a large twist it introduces a large amount of statistical error, making the effect hard to see.

Tree level calculations are done by setting the gauge field to the identity everywhere (before applying any background field or twisted boundary phase). This means that propagators can be quickly produced since there is no ensemble to average over and only one propagator per quark flavour is required. The resultant correlation functions give smooth effective masses with zero error. Of course setting the gauge field to unity turns off all QCD interactions, making the calculation a poor representation of a physical hadron. Despite this we can take advantage of the lack of statistical error to draw some meaningful conclusions.

The following tree level calculations were calculated on a $32^{3} \times 128$ lattice using a clover fermion action. Boundary conditions are periodic in the spatial dimensions and fixed in the time direction. Where noted we use twists in one spatial direction of $\exp (i \omega / L)$ with $\omega=2 \pi n / N_{x} N_{y}$. The twists on the 


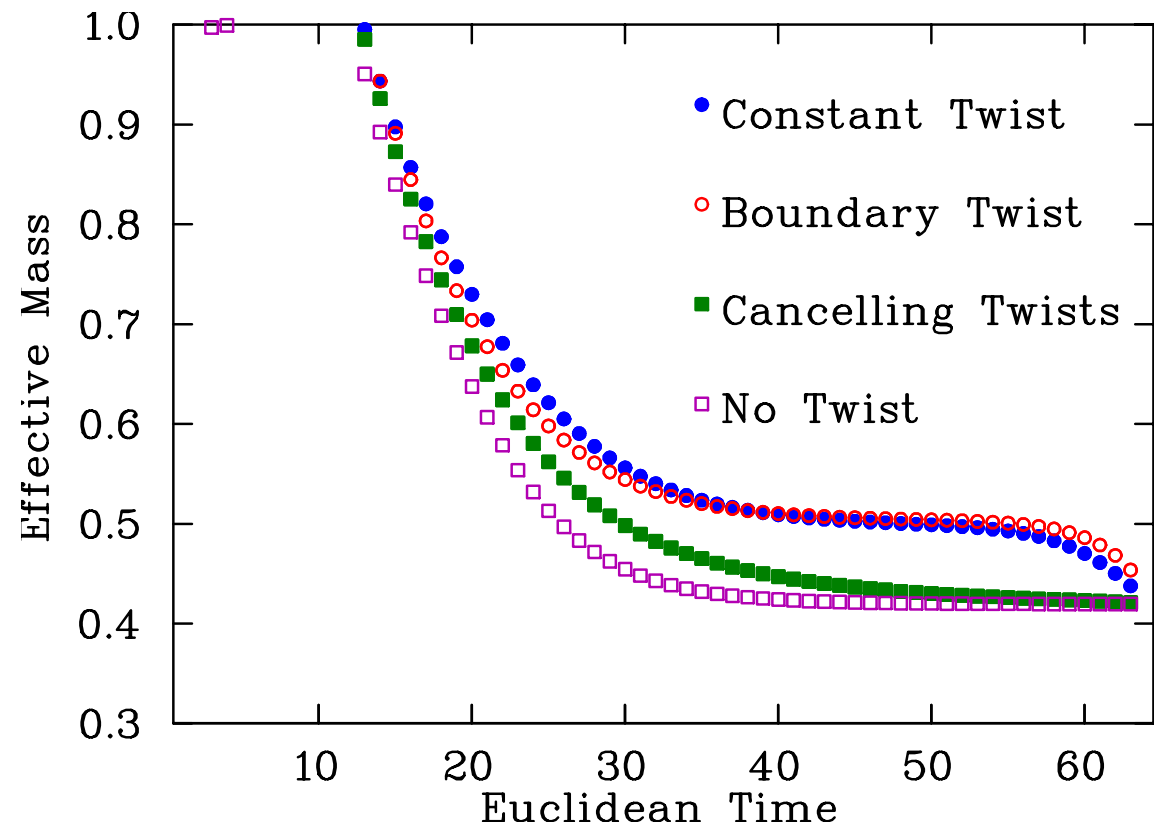

Figure 7.1: Tree level effective mass plot showing a normal neutron effective mass (no twist), an effective mass shifted by a uniform twist (constant twist), one shifted by a boundary twist, and one with a uniform twist and boundary twist designed to cancel each other out. 
up and down quark are related by their charges in the same manner as the background magnetic field. This leads to the up quarks having twice the momentum and in the opposite direction to the down quarks. For a neutral particle this results in a net momentum of zero, however the internal momenta of the quarks still leads to an increase in energy. Each correlation function uses a point sink and a source smeared with 40 sweeps of Gaussian smearing unless otherwise specified.

Figure 7.1 shows a tree level calculation of a neutron effective mass with different types of twist. The top two curves both describe twisted boundary conditions, one with a constant phase of $\exp (i \omega / L)$ on every $x$ direction link and one with the phase applied only at the $x$ boundary as one lump of phase $\exp (i \omega)$. According to the derivation of the twisted boundary conditions we expect both of these ways of applying twisted boundary conditions to cause the same increase in the neutron energy. We see that they do have the same asymptote value, although the approach to the asymptote and the large time behaviour are slightly different and this is associated with changes in the strength with which excited states are created. The lower curves are for the plain neutron mass without any twisted boundary conditions and for the case where a uniform twist and a boundary twist of the opposite sign have both been applied together. The combination of the two types of twist cancel each other, giving the same energy as for the plain neutron mass, albeit with a different decay behaviour again. In both cases, the presence of a phase throughout the lattice has enhanced the role of excited states relative to the ground state in our smeared-source local-sink correlators.

In order to test this in lattice QCD conditions we performed some calculations with twisted boundary conditions on actual gauge fields. Figure 7.2 shows fitted neutron effective mass values comparing a normal neutron mass with a neutron where each quark has been given a uniform twist proportional to its charge. The third point is a prediction for the twisted boundary condition value based on a constituent quark model. This prediction involves giving each quark a mass equal to one third of the measured neutron mass and a momentum of $-\omega / L$ for the down quarks and $2 \omega / L$ for the up quark. The total energy is then determined by a sum over the quark energies $\sum_{q} E_{q}=\sum_{q} \sqrt{p_{q}^{2}+m_{q}^{2}}$. The predicted value agrees very well with the twisted value, suggesting that the effect of the uniform twist is what we expect it to be.

This result gives new insight into the way in which finite volume effects alter the masses of hadrons. The finite volume discretises the momenta 


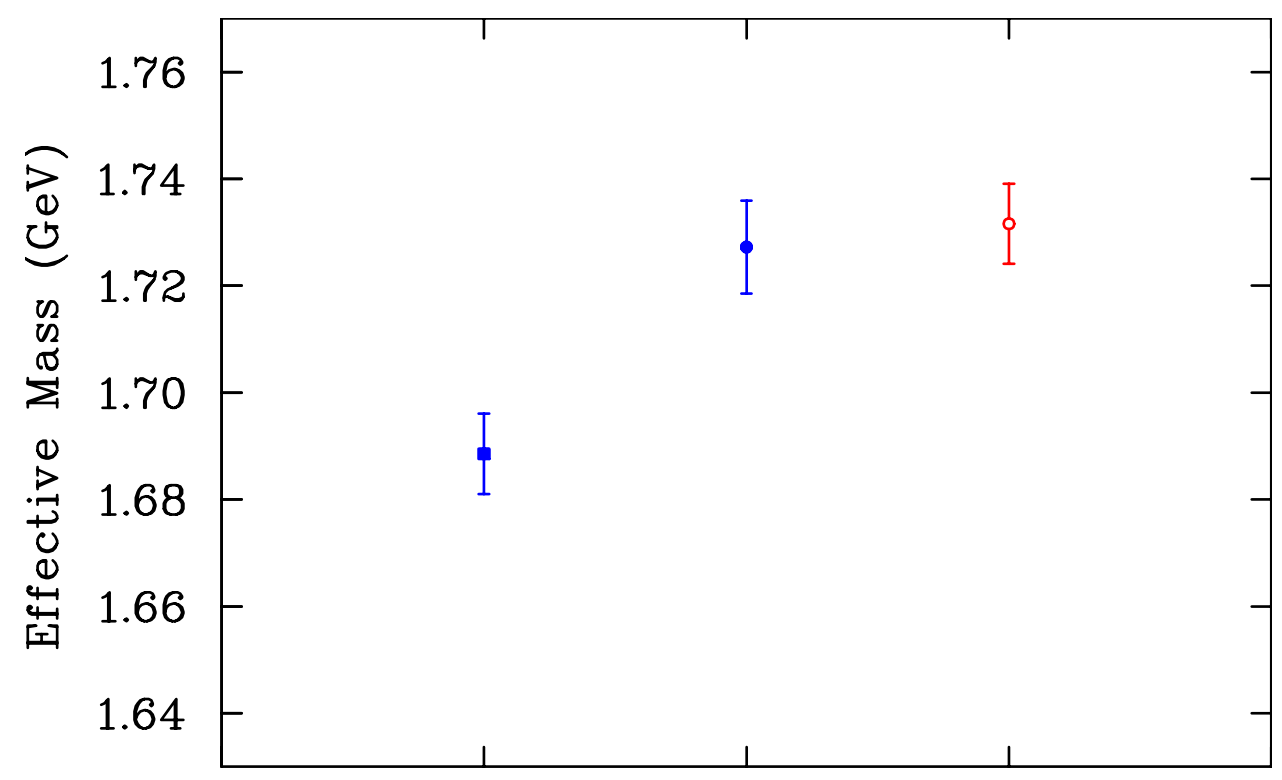

Figure 7.2: Fitted neutron effective mass values. The left point is the neutron mass with periodic boundary conditions. The middle point is the mass shifted by the addition of a uniform twist on the lattice. The right point is a simple quark model prediction for the energy change based on the expected shift in the minimum momentum for each quark from the twist. 
available to the quarks as they compose the hadrons. In the case of twisted boundary conditions, the important $\vec{p}=0$ momentum is unavailable and the true ground state is modeled poorly. However, as $V \rightarrow \infty$, the momenta needed will become available and the mass will drop. Similar effects are ongoing in the periodic boundary condition case where gaps in the available momenta force quarks into higher momentum states, thus increasing their energy on the finite volume. We note this quark-model based insight is complementary to effective field theory insights into finite-volume effects where gaps in the available momenta tend to suppress otherwise attractive meson loop contributions.

We also performed QCD calculations using a boundary twist expected to give the same momentum shift. This resulted in very large statistical errors which caused the point to be consistent with both the original mass and the uniformly twisted boundary condition mass, making the effect inconclusive. Calculating the energy with a uniform twist and a large boundary twist designed to cancel the uniform twist also had these very large errors. It seems that having a large discontinuity in the phase of the gauge field at the boundary causes instability in the calculation which leads to large statistical variation.

\subsection{Twist-like phases with a background field}

Next we examined tree level calculations which combine our quantised uniform background field with a twist-like phase, as in Eq. (7.3). The twistlike phase is the same as that in the previous section, proportional to $\omega=$ $2 \pi n / N_{x} N_{y}$ multiplied by the quark charge. We found that in tree level background field calculations using the neutron interpolator $\chi_{1}=\left(d^{T} C \gamma_{5} u\right) d$ the up and down quark in the parentheses formed a neutral scalar diquark which did not "feel" the background field. The measured magnetic moment was simply that of the remaining down quark.

Figure 7.3 shows some tree level neutron effective masses, including some modified by either a constant $U(1)$ phase, a background magnetic field, or both. The dip at the right hand end of some curves is simply an effect of the fixed time boundary. The top curve is for a pure twist of a magnitude proportional to our magnetic field quanta with $n=3$, shifting the momenta by $\omega / L=3\left(2 \pi / N_{x} N_{y}\right)$ for the down quarks and $\omega / L=-2 \cdot 3\left(2 \pi / N_{x} N_{y}\right)$ on the up quark. This is equal to the phase which is introduced by choosing $A_{x}=$ 

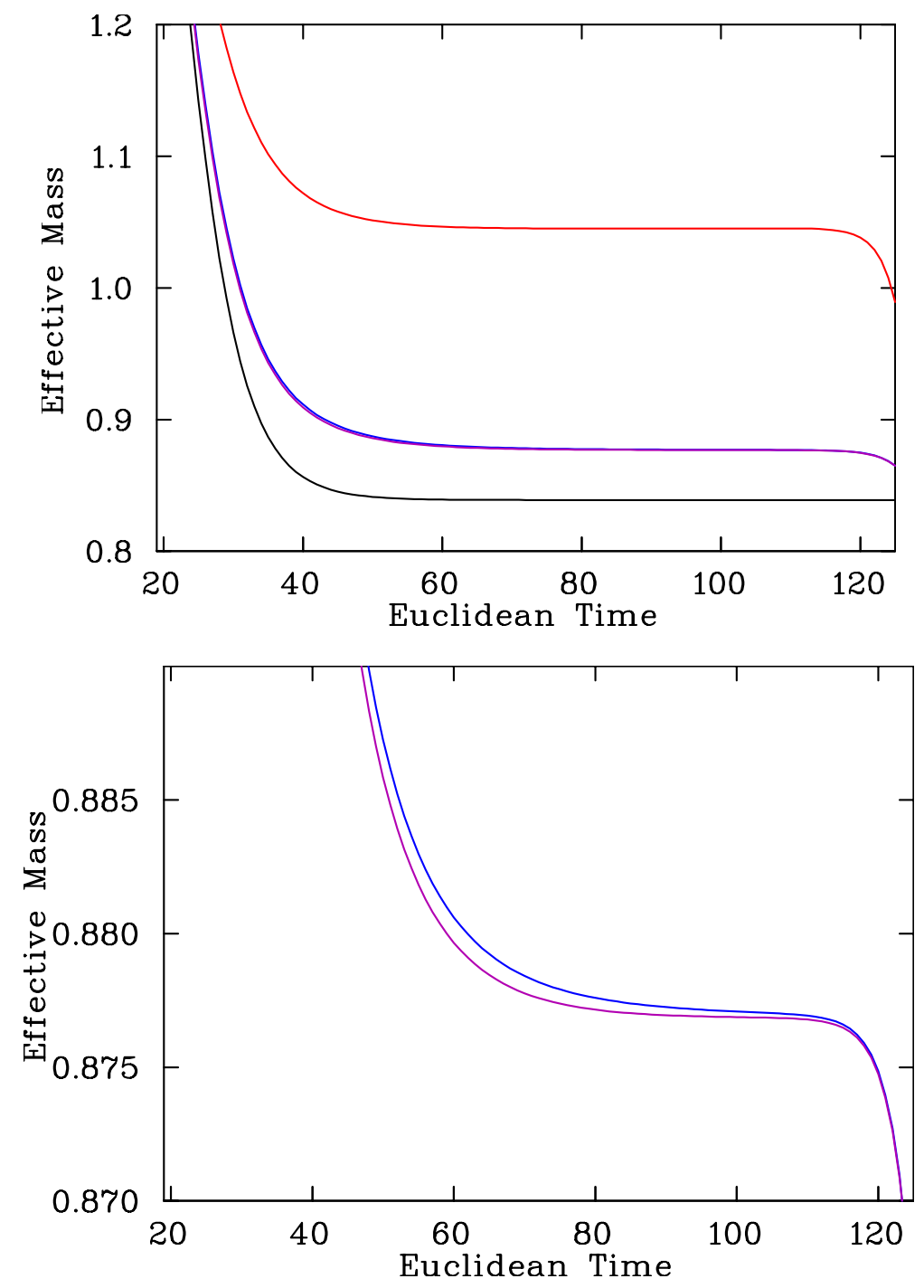

Figure 7.3: Above: Neutron effective masses in background magnetic fields and twisted boundary conditions. The bottom curve is the neutron effective mass and the top curve is the effective mass with twisted boundary conditions (in the form of a uniform $U(1)$ phase). In the middle are two curves which are almost on top of each other, one for the neutron in a background magnetic field, and one with both a magnetic field and a constant $U(1)$ phase.

Below: The same curves as above with the scale zoomed in. The bottom curve is for a neutron in a background magnetic field and the top curve is for both a magnetic field and a constant $U(1)$ twist. 
$-B(y-3)$ for our background gauge field. The resultant net momentum is zero, however the quarks are required to move in opposite directions, causing the total energy of the neutron to be increased by a significant amount. The two curves in the middle, above the bare mass curve, are almost degenerate. These are the curves for the energy in a background magnetic field and for the combination of the magnetic field and the $U(1)$ phase used in the case of the first curve. Clearly the phase has not shifted the energy level in the same way that it did without the background magnetic field present.

Figure 7.3 also has a plot of the same effective masses so we see the same nearly degenerate curves at a closer scale. Here we can tell that the curve with the twist comes in slightly higher than the one with just the magnetic field. It appears that the two curves are approaching the same asymptotic value, but due to the lack of statistical error they are true asymptotes and never actually reach that value. This means that the presence of the twistlike phase has changed the excited state overlap of the correlation function without changing the energy level of the ground state.

Since the choice of background gauge potential changes the overlap of states with the smeared interpolators, we want to know which choice gives the best behaviour. The only choice that seems "special" is the one where the constant phase term is zero. We found the most rapid approach to plateau behaviour in the effective mass occurred when the origin of the Gauge potential coincides with the origin of the propagator. Non-trivial phases in these links give rise to state overlaps favouring excited states over the desired ground state. We also tested this by moving the origin of the field via a constant phase and then putting the quark origin at the same point. We found that this did indeed give the best behaviour in terms of the flatness of the effective mass approach to the asymptote in our tree level calculations.

The previous figures have all been from propagators calculated with 40 sweeps of gauge-invariant Gaussian smearing, including the non-trivial $U(1)$ phases demanded by gauge invariance. We chose this source smearing because it is what we had been using in calculating our non-tree level quenched results. Since the $U(1)$ phase changes only the overlap of a background field correlation function we thought to try a point source and verify no sensitivity to the origin of the gauge potential. Figure 7.4 compares a background magnetic field effective mass with a magnetic field plus twist-like phase effective masses where both use a point source. The two curves are found to be completely degenerate, meaning that the $U(1)$ phase has zero effect on background field propagators that use a point source and sink. It is the smearing 


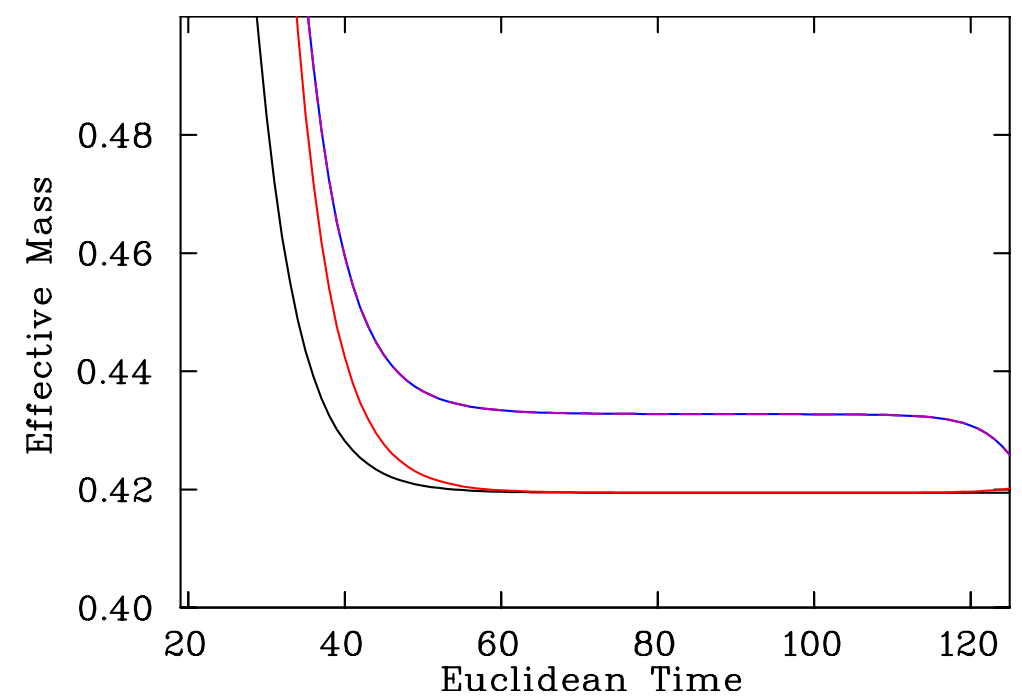

Figure 7.4: Tree level neutron effective masses. The left most curve is the mass with 40 sweeps of fermion source smearing. The curve which comes down to meet the first curve is again the mass but using a point source. The top curve is actually two degenerate curves, one for the neutron in a background magnetic field and the other for a magnetic field and a constant $U(1)$ twist-like phase, both using a point source. 
of the fermion source that allows the $U(1)$ phase to change the strength of the states participating in the correlation functions. We note the twist-like phase does not alter the spectrum. Only the overlap of excited states is changed in the correlation function.

\subsubsection{Explanation}

We have seen that twisted boundary conditions have no effect when introduced with a uniform background magnetic field so long as they both use the quantised phase term $\omega=2 \pi n / N_{x} N_{y}$ with $n$ an integer. This can be explained by making use of a two-dimensional $4 \times 4$ lattice to demonstrate. We use our usual background magnetic field defined on a periodic lattice,

$$
\begin{gathered}
U_{1}(x, y)=\exp (-i \omega y) . \\
U_{2}(x, y)=\left\{\begin{array}{cc}
\exp \left(+i \omega n_{y} x\right), & y=\left(n_{y}-1\right) \\
1, & \text { elsewhere }
\end{array}\right.
\end{gathered}
$$

which we modify by adding a constant twist across the whole lattice

$$
U_{1}^{\prime}(x, y)=\exp (-i \omega y) \exp (i \omega)=\exp (-i \omega(y-1))
$$

This is equivalent to shifting the origin of the gauge potential by one lattice site, as seen in Figure $7.5 \mathrm{a}$ ) and b), from $y=0$ to $y=1$. We then apply a gauge transformation which we define,

$$
G(x, y)=\left\{\begin{array}{cc}
\exp (+i \omega 4 x), & y=0 \\
1, & \text { elsewhere }
\end{array}\right.
$$

This is the identity over most of the lattice, but changes the $U_{1}(x, y)$ values along the bottom row,

$$
\begin{aligned}
U_{1}^{\prime}(x, 0) & =G(x, 0) U_{1}(x, 0) G^{\dagger}(x+1,0) \\
& =\exp (4 i \omega x) \exp (i \omega) \exp (-4 i \omega(x+1)) \\
& =\exp (-3 i \omega)
\end{aligned}
$$

except at the boundary $x=n_{x}-1=3$ where we get,

$$
\begin{aligned}
U_{1}^{\prime}(3,0) & =G(3,0) U_{1}(3,0) G^{\dagger}(0,0) \\
& =\exp (12 i \omega) \exp (i \omega) \exp (0)) \\
& =\exp (13 i \omega)=\exp (-3 i \omega) \exp (16 i \omega)
\end{aligned}
$$


The boundary part of $U_{2}(x, y)$ is also changed with the top row going to 1 ,

$$
\begin{aligned}
U_{2}^{\prime}(x, 3) & =G(x, 3) U_{2}(x, 3) G^{\dagger}(x, 0) \\
& =\exp (0) \exp (4 i \omega x) \exp (-4 i \omega x) \\
& =\exp (0)=1
\end{aligned}
$$

while the $U_{2}(x, y)$ for the bottom row is changed to what the top row used to be,

$$
\begin{aligned}
U_{2}^{\prime}(x, 0) & =G(x, 0) U_{2}(x, 0) G^{\dagger}(x, 1) \\
& =\exp (4 i \omega x) \exp (0) \exp (0)) \\
& =\exp (4 i \omega x)
\end{aligned}
$$

This results in Figure $7.5 \mathrm{c}$ ), which is almost the same as a) but with the top row at the bottom and an unwanted term in $U_{1}(3,0)$. We then take advantage of the periodic boundary conditions to simply take the bottom row and put it at the top, giving us Figure $7.5 \mathrm{~d}$ ).

In order to make d) look like a) we need to get rid of the $\exp (16 i \omega)$ term in $U_{1}(3,0)$. Fortunately this is extremely easy when we just look at the definition of $\omega$. Due to the periodic boundary conditions there is a quantisation condition on the allowed values of $\omega$,

$$
\omega=\frac{2 \pi n}{N_{x} N_{y}}
$$

where $n$ can be any integer. For our $4 \times 4$ demonstration we have $N_{x}=N_{y}=$ 4 , which means the unwanted term is,

$$
\exp (16 i \omega)=\exp (16 i 2 \pi /(4 \cdot 4))=\exp (i 2 \pi)=1
$$

Therefore the unwanted term disappears due to the "magic" of our quantisation condition. This leaves us with exactly the field we started with, before adding the constant phase. Thus, the phase has no effect. This holds for larger lattices using the same quantisation condition and explains why we do not see any change in the effective mass when a constant twist-like phase is added to a background field calculation. For other background field calculations which do not have the same quantisation condition then twisted boundary conditions could still be a significant concern, in addition to the errors from avoiding the quantisation. 
a)

$$
\begin{array}{lrrrrrrr}
0 & & 4 i \omega & 8 i \omega & \multicolumn{2}{c}{12 i \omega} \\
3,0 & -3 i \omega & 3,1 & -3 i \omega & 3,2 & -3 i \omega & 3,3 & -3 i \omega \\
0 & 0 & 0 & 0 & \\
2.0 & -2 i \omega & 2,1 & -2 i \omega & 2,2 & -2 i \omega & 2,3 & -2 i \omega \\
0 & & 0 & & 0 & & 0 & \\
1,0 & -1 i \omega & 1,1 & -1 i \omega & 1,2 & -1 i \omega & 3,3 & -1 i \omega \\
0 & & 0 & & 0 & & 0 & \\
0 & 0 & 1,0 & 0 & 2,0 & 0 & 3,0 & 0
\end{array}
$$

c)

$\begin{array}{lrrrrrrr}0 & & 0 & 0 & 0 & \\ 3,0 & -2 i \omega & 3,1 & -2 i \omega & 3,2 & -2 i \omega & 3,3 & -2 i \omega \\ 0 & 0 & 0 & 0 & \\ 2.0 & -1 i \omega & 2,1 & -1 i \omega & 2,2 & -1 i \omega & 2,3 & -1 i \omega \\ 0 & & 0 & & 0 & & 0 & \\ 1,0 & 0 & 1,1 & 0 & 1,2 & 0 & 1,3 & 0 \\ 0 & & 4 i \omega & & 8 i \omega & & 12 i \omega & \\ 0 ., 0 & -3 i \omega & 1,0 & -3 i \omega & 2,0 & -3 i \omega & 3,0 & 13 i \omega\end{array}$

b)

$$
\begin{aligned}
& 0 \quad 4 \mathrm{i} \omega \quad 8 \mathrm{i} \omega \quad 12 \mathrm{i} \omega \\
& \text { (3,0 }-2 \mathrm{i} \omega \text { (3,1 }-2 \mathrm{i} \omega \text { (3,2 }-2 \mathrm{i} \omega \text { (3,3 }-2 \mathrm{i} \omega \\
& \begin{array}{llll}
0 & 0 & 0 & 0
\end{array}
\end{aligned}
$$$$
\text { (2.0) }-1 i \omega \text { 2,1 }-1 i \omega \text { 2,2 }-1 i \omega \text { (2,3) }-1 i \omega
$$$$
\begin{array}{llll}
0 & 0 & 0 & 0
\end{array}
$$

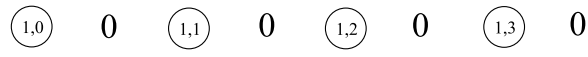$$
\begin{array}{llll}
0 & 0 & 0 & 0
\end{array}
$$$$
\text { (0,0) } \quad 1 \mathrm{i} \omega \text { (1,0 } 1 \mathrm{i} \omega \text { 2,0 } 1 \mathrm{i} \omega \text { 3,0 } 1 \mathrm{i} \omega
$$

d)

$$
\begin{aligned}
& \begin{array}{llll}
0 & 4 \mathrm{i} \omega & 8 \mathrm{i} \omega & 12 \mathrm{i} \omega
\end{array} \\
& \text { (3.0) }-3 i \omega \text { (3,1) }-3 i \omega \text { (3,2) }-3 i \omega \text { (3,3) }-3 i \omega 16 i \omega \\
& \begin{array}{llll}
0 & 0 & 0 & 0
\end{array} \\
& \text { (2.0) }-2 \mathrm{i} \omega \text { (2,1) }-2 \mathrm{i} \omega \text { (2,2) }-2 \mathrm{i} \omega \text { (2,3) }-2 \mathrm{i} \omega \\
& \begin{array}{llll}
0 & 0 & 0 & 0
\end{array} \\
& \text { (1.0) }-1 \mathrm{i} \omega \text { (1.1) }-1 \mathrm{i} \omega \text { (1.2) }-1 \mathrm{i} \omega \text { (1,3) }-1 \mathrm{i} \omega \\
& \begin{array}{llll}
0 & 0 & 0 & 0
\end{array} \\
& \begin{array}{llllllll}
0,0 & 0 & 1,0 & 0 & 2,0 & 0 & 3,0 & 0
\end{array}
\end{aligned}
$$

Figure 7.5: Diagram showing the effect of a constant phase on a $4 \times 4$ lattice and how it can be gauge transformed away. The $(x, y)$ coordinates are shown for each lattice site and the exponents of the $U(1)$ links are shown between them. a) shows a standard background field implementation with the gauge potential origin at $(0,0)$. b) shows the field shifted by the constant phase $e^{i \omega}$ in the $x$ direction. c) shows the effects of the gauge transformation. d) has the coordinates shifted via periodic boundary conditions to put the bottom row at the top. 


\subsection{Summary}

In this section we have shown that the choice of gauge potential for creating a background magnetic field can have an effect on the relative overlap of excited states in the correlation function. The physical states, however, are not affected. This has been verified using tree level calculations of background field correlation functions with a twist-like phase added. It has also been shown explicitly through a description of how a gauge transformation and periodic boundary conditions can be used to remove the phase completely.

The changes to the excited state spectrum are due to phases introduced to the smeared fermion source. When using a point source the addition of a twist-like phase has no effect on the calculation. When using a smeared source the effect of the phase on the excited state overlap is minimized when

the origin of the gauge field and the origin of the quark propagators is the same. 


\section{Chapter 8}

\section{Magnetic Moment}

\subsection{Introduction}

The magnetic moment is one of the most fundamental electromagnetic properties of a system. There are a number of definitions depending on the type of system that is being described. One of the simplest definitions is that associated with a planar loop of current,

$$
\boldsymbol{\mu}=I \mathbf{S},
$$

where $\mu$ is the magnetic moment, $I$ the current and $S$ the surface area enclosed by the loop. The direction of the moment is conventionally given by the right hand rule. This definition can be generalised to the case of an arbitrary distribution of current $\mathbf{J}$,

$$
\boldsymbol{\mu}=\frac{1}{2} \int \mathbf{r} \times \mathbf{J} \mathrm{dV},
$$

where $\mathbf{r}$ is a vector pointing from the origin to the location of the volume element $\mathrm{dV}$. These are actually generalised cases of a single charged particle moving along a circular path, for which the magnetic moment is

$$
\boldsymbol{\mu}=\frac{1}{2} q \mathbf{r} \times \mathbf{v},
$$

where $\mathbf{r}$ is the position of the charge relative to the centre of the circle and $\mathbf{v}$ is the velocity of the charge. From this we see that magnetic moments are closely bound with angular momentum. 
The moment can also be seen as a measurement of the strength and direction of the torque induced on a system in the presence of an externally applied magnetic field,

$$
\tau=\boldsymbol{\mu} \times \mathbf{B},
$$

where $\tau$ is the torque and $\mathbf{B}$ is the magnetic field. This is, for example, the force which aligns the needle of a compass with the earth's magnetic field.

The magnetic moment can more accurately be called the magnetic dipole moment, the coefficient of the first term in the multipole expansion of a general magnetic field. In Nature there do not appear to exist any magnetic monopoles (particles with magnetic charge), although some theories do suggest that they could be found.

In the context of hadron physics the magnetic moment of a particle is due to contributions associated with the angular momentum of the quarks that make up the hadron. There can be contributions from not only the valence quarks but the sea quarks as well. The magnetic moment is due primarily to the intrinsic spin of the quarks. The ability of a charged particle with only spin angular momentum to produce a magnetic moment is best seen in the electron, for which the magnetic moment is clearly established.

On the lattice, in addition to the background field method, the magnetic moment can also be calculated using three point functions [31-33]. This is done by calculating electromagnetic form factors and then extrapolating to zero momentum, where the form factors can be related to the magnetic moment. This technique is effective but less direct than the background field method. We will compare our magnetic moment results with some that were calculated using the three point function method.

\subsection{Quark model prediction}

It is worth touching on the naive constituent quark model prediction of the magnetic moment for the proton and neutron. This model uses the $S U(6)$ spin-flavour representation where the only degrees of freedom are the spins (up, down) and the flavours $(u, d, s)$ of the quarks. In this representation the proton spin-up state can be written as,

$$
|p \uparrow\rangle=\frac{1}{\sqrt{18}}\left|2(u \uparrow u \uparrow d \downarrow)_{s y m}-(u \uparrow u \downarrow d \uparrow)_{\text {sym }}\right\rangle,
$$


and the neutron can likewise be written as,

$$
|n \uparrow\rangle=\frac{1}{\sqrt{18}}\left|2(d \uparrow d \uparrow u \downarrow)_{s y m}-(d \uparrow d \downarrow u \uparrow)_{\text {sym }}\right\rangle .
$$

The subscript sym indicates all symmetric combinations of the three quarks in the term. For the second term there are six combinations (three locations for the singly represented flavour and swapping the doubly represented ones) and three combinations for the first term (since the doubly represented quarks are not distinguishable). The $1 / \sqrt{18}$ term normalises the states,

$$
\langle p \uparrow \mid p \uparrow\rangle=\langle n \uparrow \mid n \uparrow\rangle=1 .
$$

To calculate the magnetic moment in the quark model we use the magnetic moment operator $\hat{\mu}$ which acts on the states to pull out the contribution of each quark.

$$
\begin{aligned}
& \langle p \uparrow|\hat{\mu}| p \uparrow\rangle \\
= & \frac{1}{18}\left(4\langle u \uparrow u \uparrow d \downarrow|\hat{\mu}| u \uparrow u \uparrow d \downarrow\rangle_{\text {sym }}+\langle u \uparrow u \downarrow d \uparrow|\hat{\mu}| u \uparrow u \downarrow d \uparrow\rangle_{\text {sym }}\right) \\
= & \frac{1}{18}\left(4 \cdot 3\left(2 \mu_{u}-\mu_{d}\right)+6\left(\mu_{u}-\mu_{u}+\mu_{d}\right)\right) \\
= & \frac{1}{18}\left(24 \mu_{u}-12 \mu_{d}+6 \mu_{d}\right) \\
= & \frac{4}{3} \mu_{u}-\frac{1}{3} \mu_{d} .
\end{aligned}
$$

The neutron is the same except with $u$ and $d$ quarks swapped,

$$
\langle n \uparrow|\hat{\mu}| n \uparrow\rangle=\frac{4}{3} \mu_{d}-\frac{1}{3} \mu_{u} .
$$

We can then give each quark one third of the nucleon mass and use an analogy to the Dirac moment to get the moments of the quarks in terms of the nuclear magneton $\mu_{N}=\frac{e \hbar}{2 M_{N}}$,

$$
\frac{1}{2} \mu_{u}=-\mu_{d}=\mu_{N}
$$

Inserting these values into Equations (8.8) and (8.9) gives us the quark model prediction for the magnetic moments of the proton and neutron,

$$
\mu_{p}=3 \mu_{N}, \quad \mu_{n}=-2 \mu_{N} .
$$


These are close to the experimental values of $2.8 \mu_{N}$ and $-1.9 \mu_{N}$ for the proton and neutron respectively. The $S U(6)$ spin-flavour quark model provides a reasonable representation of the magnetic moments of all octet baryons. However there are fascinating discrepancies [34,35].

\subsection{Method}

The use of the background field method for calculating magnetic moments has been well established $[9,13,15,19]$. Recall the energy-field relationship for a hadron in a uniform magnetic field $[9,11]$,

$$
E(B)=M-\boldsymbol{\mu} \cdot \mathbf{B}+\frac{e|B|}{2 M}-\frac{4 \pi}{2} \beta B^{2}+\mathcal{O}\left(B^{3}\right) .
$$

In theory we could calculate an effective mass in a magnetic field and simply fit it to the four terms in this equation, however this would be very difficult and imprecise. Instead we isolate the terms we are interested in before trying to fit against the field. To calculate the magnetic moment we use the fact that the relevant term contains a dot product between the field and the magnetic moment itself. This means that the energy shift is dependent on the angle between the field and the spin of the particle.

On the lattice, where we have the spin quantised and field aligned on the z-axis, the shift has equal magnitude, but opposite sign, when the spin and field are aligned versus when they are anti-aligned. All the other terms in Eq. (8.12) are independent of the alignment of the spin and the field. This means by taking differences we can get all the terms other than the moment term to cancel out (neglecting higher order terms). This difference can be taken between either spin-up and spin-down or between the field in the positive $z$-direction and the field in the negative $z$-direction.

$$
\frac{1}{2} \delta E=\frac{1}{2}\left(E_{\uparrow}(B)-E_{\downarrow}(B)\right)=-\mu B .
$$

In the ensemble average these two approaches are identical. Since each correlation function already comes to us with spin-up and spin-down separate, it is more efficient for us to use a difference of spins. Using positive and negative fields does give somewhat higher statistics, but this is roughly equivalent to doing extra fermion sources for the same cost.

There are a number of ways of taking the difference of spins to isolate the magnetic moment term from Eq. (8.12), but some are more effective than 
others. The simplest is to just fit spin-up and spin-down and then subtract one from the other.

$$
\Delta E(B)=\left(\left(G_{\uparrow}(B, t)\right)_{f i t}-\left(G_{\downarrow}(B, t)\right)_{f i t}\right) / 2,
$$

where the subscript ${ }_{\text {fit }}$ implies a fit to the effective mass as described in Eq. (3.30) or effective energy in the case of combinations of correlation functions. However this provides little insight into the appropriate fit windows for obtaining the best measure of $\Delta E$. The uncertainties in $G_{\uparrow}$ and $G_{\downarrow}$ are correlated and it is essential to take advantage of this.

Another way is to construct a ratio of spin-up and spin-down correlation functions before fitting for the energy shift,

$$
\Delta E(B)=\frac{1}{2}\left(\frac{G_{\uparrow}(B, t)}{G_{\downarrow}(B, t)}\right)_{f i t} .
$$

This method allows for fluctuations in the correlation function which are independent of the spin to cancel. This results in smaller errors in the spin difference value, ranging from around $2 \mathrm{MeV}$ to $4 \mathrm{MeV}$ as opposed to 6 to $15 \mathrm{MeV}$ for each of the correlators.

The fit can be improved further by recognising that many of the fluctuations are not affected by the presence of the background field. This can be seen in Figure 8.1, which shows spin-up effective mass values with and without a magnetic field. Although each line has small ups and downs they are almost completely parallel to each other, at least up until the later times when noise is intruding. This means we can cancel out a lot of correlated errors by subtracting off the zero field mass explicitly. This can be achieved using the construct,

$$
\Delta E(B)=\frac{1}{2}\left(\left(\frac{G_{\uparrow}(B, t)}{G_{\uparrow}(0, t)}\right)_{f i t}-\left(\frac{G_{\downarrow}(B, t)}{G_{\downarrow}(0, t)}\right)_{f i t}\right),
$$

but again this can be improved by combining the difference into one ratio,

$$
\Delta E(B)=\frac{1}{2}\left(\frac{G_{\uparrow}(B, t)}{G_{\uparrow}(0, t)} \frac{G_{\downarrow}(0, t)}{G_{\downarrow}(B, t)}\right)_{f i t} .
$$

Using this form allows for correlated errors to cancel between spin-up and spin-down as well as field on and field off. It requires only a single fit and results in the smallest possible errors, with some as low as half an MeV. This provides us with strong constraints on the fit regime. In addition, this construction constrains $\Delta E=0$ exactly at $B=0$. 


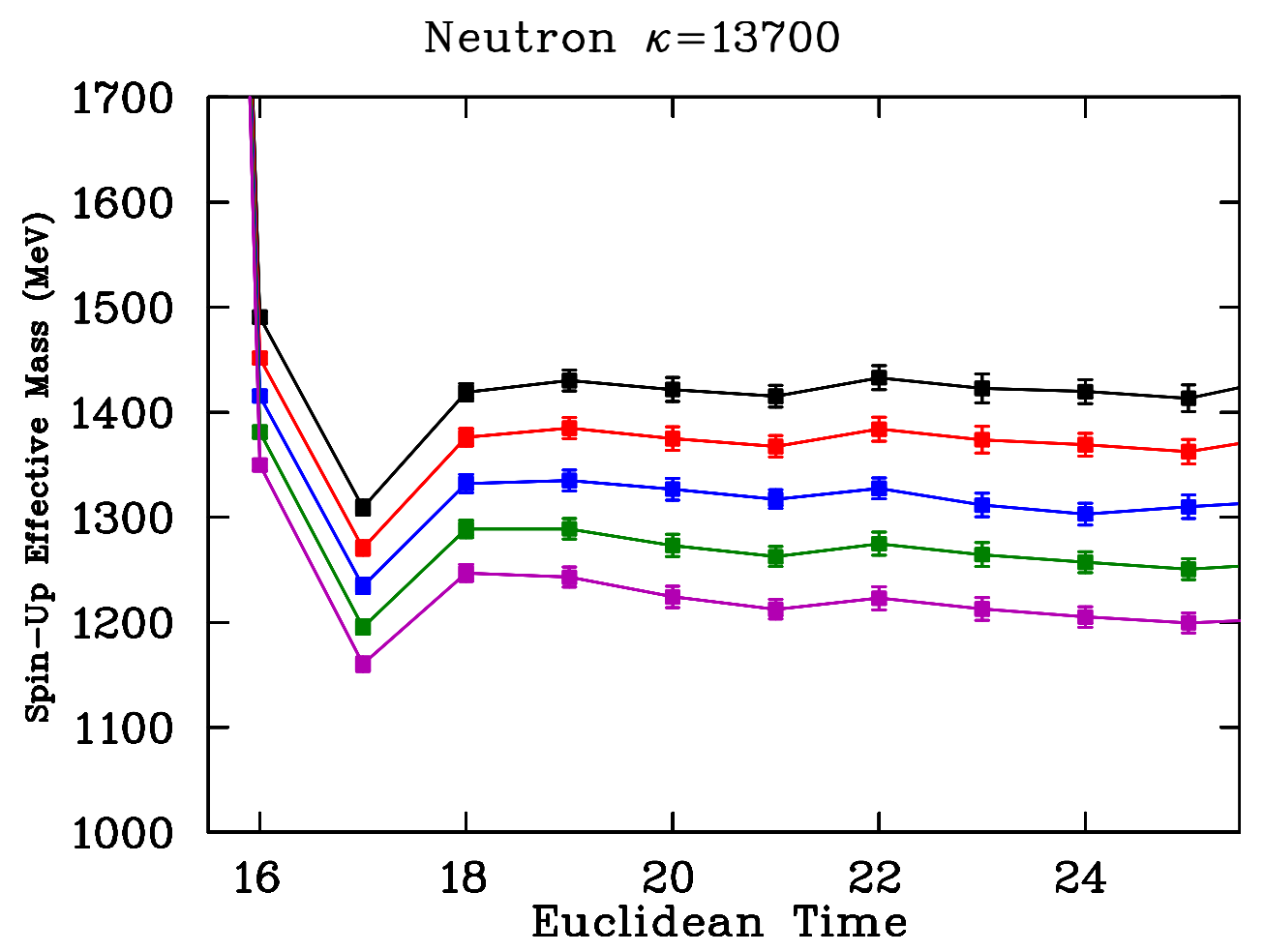

Figure 8.1: Neutron spin-up effective mass plot. Each line represents a different background field strength from zero field at the top to the largest field at the bottom. The source is at $t=16$. 


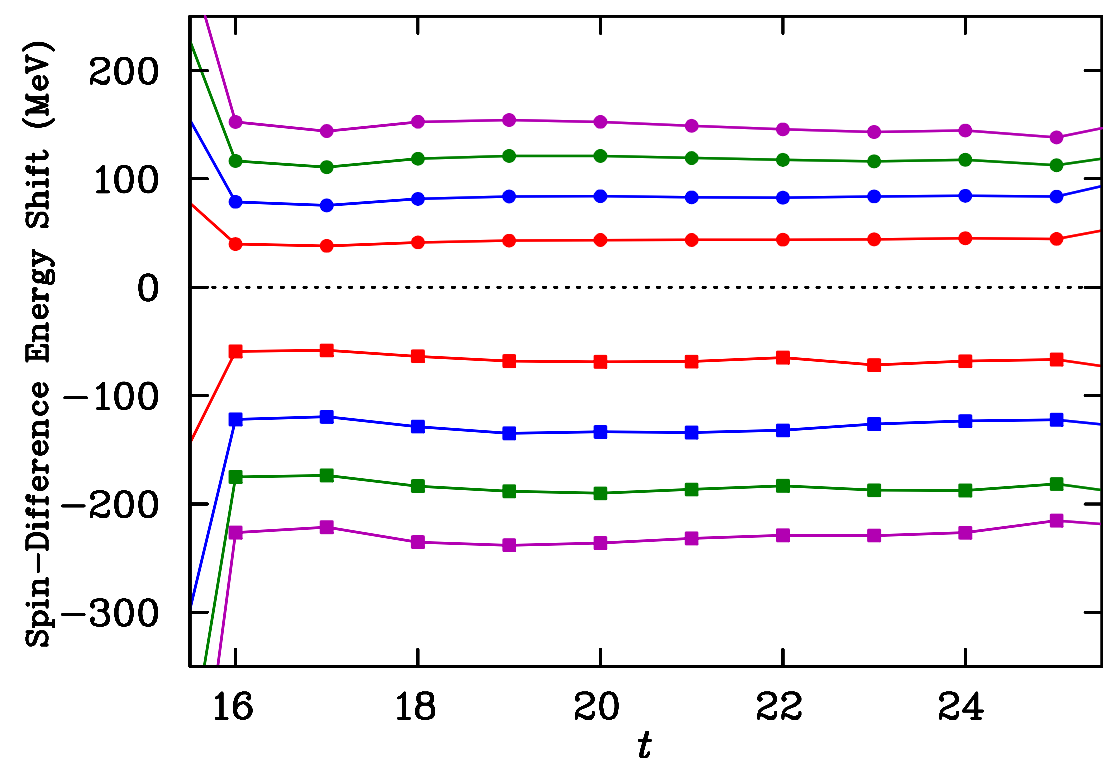

Figure 8.2: Spin-difference effective energy plot from Eq. (8.17) at $\kappa=$ 0.13700 where $m_{\pi}=622 \mathrm{MeV}$. The circles are for the neutron and the squares are for the proton. The energy shift increases in magnitude with field strength.

\subsection{Results}

\subsubsection{Effective energies}

Using the ratio described in Eq. (8.17) we construct effective measures for $\Delta E$ from the difference of spins. In order to extract a value for the energy shift we have to find plateaus in the effective energy and fit to a constant in that time window. We judge our fits based on the criteria of the $\chi_{\text {dof }}^{2}$ of the fit and the size of the errors. We use a covariant $\chi^{2}$ as described in Eq. 5.8. To minimize the uncertainties we select the earliest possible fit window that results in an acceptable $\chi_{\text {dof }}^{2}$ value. We constructed an algorithm which attempts to chose a fit window automatically by following this approach, trying to choose a window that would be acceptable at all the field strengths for a given mass. Although this automated approach was effective much of the time, we never totally got past the need to examine the graphs and associated $\chi_{\text {dof }}^{2}$ values.

Figures 8.2 to 8.5 show these spin-difference energy shifts at various field strengths obtained via Eq. (8.17) for each of the quark masses considered. 


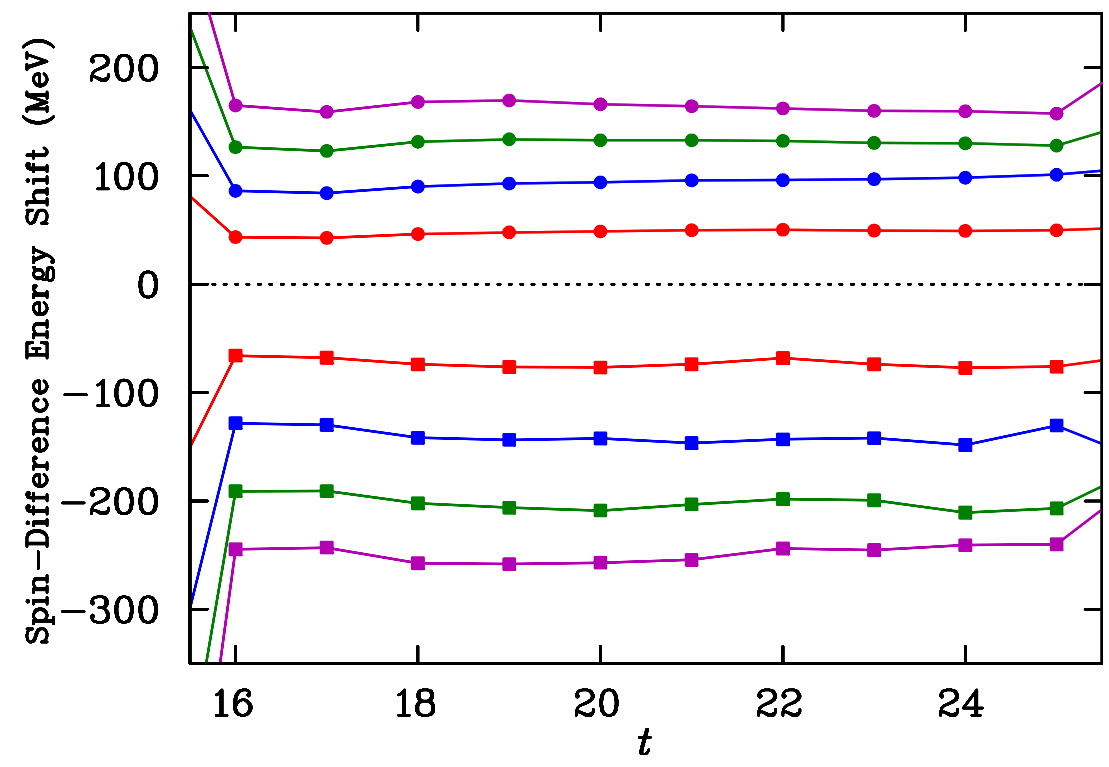

Figure 8.3: Spin-difference effective energy plot from Eq. (8.17) at $\kappa=$ 0.13727 where $m_{\pi}=512 \mathrm{MeV}$. The circles are for the neutron and the squares are for the proton. The mass shift increases in magnitude with field strength.

Usually in lattice calculations we have to wait for Euclidean time evolution to kill off the excited state contributions, however we see that there is very little excited state effect apparent for both the proton and neutron, with the lines coming in flat right from the source. In general the spin-difference energy shift curves are very flat and we immediately see that there is an approximately linear progression in the shifts. This matches what we would expect from the linearly increasing magnetic field strengths and the linear dependence of the magnetic moment term in the energy shift on the field. It also appears that the neutron effective energies are generally flatter than those for the proton and have smaller errors, even taking into account the smaller magnitude of the neutron's shifts. This may be due to the effect of there not being a proper zero momentum state to project onto for the proton as described in Chapter 6. The errors get larger and the noise at late times becomes more significant as we go to lighter quark masses, as is typical for lattice calculations. At the lightest quark mass the proton effective energies become quite noisy, with some of the noise appearing to affect points as early as time slice 21. All other masses look good well into the range where we 


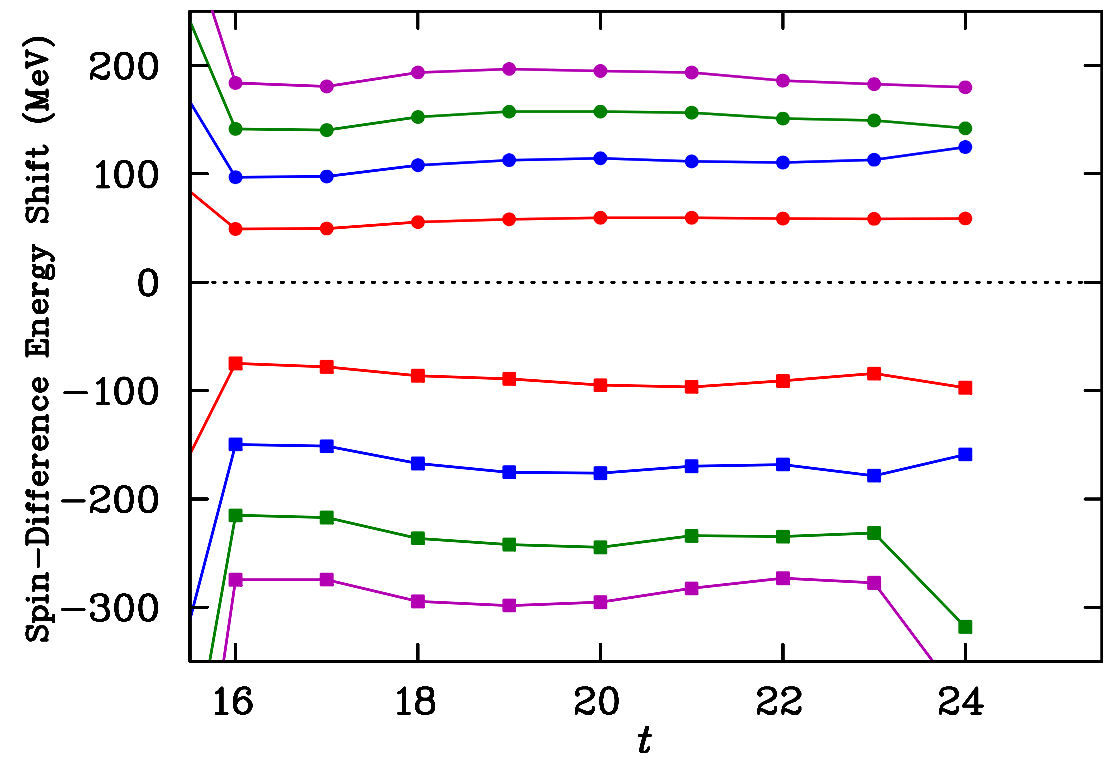

Figure 8.4: Spin-difference effective energy plot from Eq. (8.17) at $\kappa=$ 0.13754 where $m_{\pi}=388 \mathrm{MeV}$. The circles are for the neutron and the squares are for the proton. The mass shift increases in magnitude with field strength.

want to be fitting.

We observe that the effective energy curve appears to be flatter at the two smaller field strengths. The two larger field strengths still give plateaus which can be easily fit, however these plateaus are not as long as for the smaller fields. There are also cases where the larger field strengths have a section which is totally flat, then a slight drift in the curve, and then another plateau. This leads to ambiguity as to where in Euclidean time we should fit. As stated above we tend to fit as early as possible so long as the $\chi_{\text {dof }}^{2}$ is good. Since plateaus are flat the $\chi^{2}$ 's are great, but the later plateaus are just as good and have about the same error. It is likely that the plateaus at earlier times are affected by some small excited state contributions which change their value. Therefore we choose to fit at the later plateaus. We also restrict ourselves to always fitting in the same window for each field strength and quark mass. This ensures that we do not introduce systematic bias between the values by picking and choosing the best windows in each separate case.

Despite the drift over time in the effective energies for the higher field strengths the value of the magnetic moment is not actually very dependent 


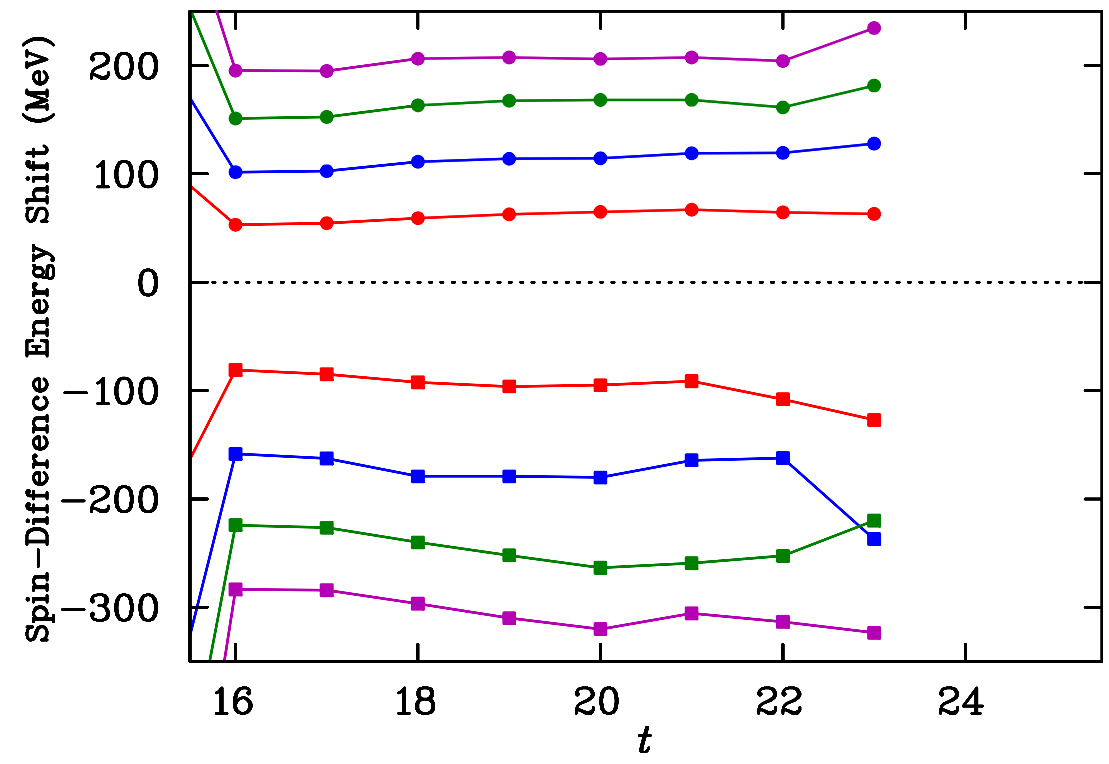

Figure 8.5: Spin-difference effective energy plot from Eq. (8.17) at $\kappa=$ 0.13770 where $m_{\pi}=282 \mathrm{MeV}$. The circles are for the neutron and the squares are for the proton. The mass shift increases in magnitude with field strength.

on the choice of fit window. The reasons for this are described in the next subsection, but we can demonstrate that this is true by redoing the calculation with a number of different fit windows. Table 8.1 contains magnetic moment values for the neutron at a number of different fit windows. We see that at every quark mass there is not a lot of change in the magnetic moment values as the fit window is changed. The only values that don't agree within errors are the ones from the earliest fit window, and that is only because the errors in general are very small. This suggests that time slice 19 may have just a little bit of excited state contamination, making it too early to fit. The smallest errors at each quark mass come from the 20-22 and 20-24 fit windows. This is probably because they start earlier than the other windows, where the errors in the effective energy are slightly smaller. Although these two fit windows agree almost perfectly in terms of effective energy fits and moment values, the shorter window has better $\chi_{\text {dof }}^{2}$ values $\sim 1$, so we use that as our window for all the spin-difference energy shift fits. 


\begin{tabular}{c|cccc}
\hline \hline & \multicolumn{4}{|c}{$\kappa$} \\
Window & 0.13700 & 0.13727 & 0.13754 & 0.13770 \\
\hline $19-21$ & $-1.187(12)$ & $-1.300(13)$ & $-1.420(16)$ & $-1.486(36)$ \\
$20-22$ & $-1.194(11)$ & $-1.317(15)$ & $-1.462(22)$ & $-1.483(30)$ \\
$21-23$ & $-1.198(13)$ & $-1.338(20)$ & $-1.454(27)$ & $-1.500(40)$ \\
$22-24$ & $-1.201(15)$ & $-1.343(25)$ & $-1.454(32)$ & $-1.508(49)$ \\
$20-24$ & $-1.199(10)$ & $-1.321(15)$ & $-1.462(20)$ & $-1.485(31)$ \\
\hline \hline
\end{tabular}

Table 8.1: Magnetic moment values for the neutron in units of nuclear magnetons $\left(\mu_{N}\right)$ at each $\kappa$ value for a variety of fit windows.

\subsubsection{Magnetic field dependence}

Once we have fit our spin-differenced effective energy shifts we must then fit against the field strength. This allows us to isolate the magnetic moment value from the $\boldsymbol{\mu} \cdot \mathbf{B}$ mass shift. Thanks to the way we calculate our spindifference in Eq. (8.17) the zero field point is exactly zero by construction. Therefore we can attempt to fit our mass shift using only a single parameter fit. The result of trying to fit a purely linear function to all four points was unacceptably large $\chi_{\text {dof }}^{2}$ values. This is because the data points do not follow a perfectly linear relationship with the field. We see evidence of this fact even in the energy shift plots, where the difference between the plateaus at each successive magnetic field strength gets noticeably smaller each time. This is an effect of the higher order terms described in the energy-field relation of Eq. (8.12). What this says is that the larger field strengths we are using are somewhat too large, making the higher order terms in $B$ non-negligible.

In order to take into account the higher order contributions at the larger field strengths we need to add extra terms into our fit. We know from Eq. (8.12) that the quadratic term in $B$ is cancelled out by the spin-difference, so the next polynomial term we can add is a $B^{3}$ term. We perform our least squares fit of the four field strengths to the function,

$$
\Delta E(B)=a B+b B^{3},
$$

which allows us to fit all four points comfortably. This fit is shown by the dashed line in Figures 8.7 and 8.6. The coefficient for $b$ in Eq. (8.18) is allowed to vary freely and results in quite a large value with significant error. Since $B^{3}$ is still fairly small at the largest field strengths the coefficient has to be large to have any noticeable effect. The value of the linear coefficient, from 
which the magnetic moment is derived, is not affected much by the variations in the cubic coefficient. This means that the energy shift measured at the two largest field strengths can vary quite significantly and the difference will be absorbed into the cubic coefficient without affecting the magnetic moment result. This is the reason for the relative lack of fit window dependence we found in the previous subsection. It also tells us that the two smaller field strength points are the main drivers of the magnetic moment value.

Since the first two points are the most important ones for determining the magnetic moment it is prudent to perform a fit that focuses on them alone. We use a one parameter linear fit on these points since we expect the higher order contributions to be negligible at the small field strengths. This allows us to check that the cubic term we introduced to fit the larger field strength points is not unduly affecting our magnetic moment result. The linear fit to the first two points can be seen as the solid line in Figures 8.7 and 8.6. The magnetic moment value coming from the linear coefficient agrees very well with that from the cubic fit. Although the linear fit looks good to the eye it has larger $\chi_{\text {dof }}^{2}$ values. This is because of the extremely small errors in the spin-difference energy shift fits, which are only about $1 \%$, so even a very small amount of higher order effect is enough to give large $\chi^{2}$ 's. A comparison of the fit values and $\chi_{\text {dof }}^{2}$ values for the neutron using 2 points and 1 parameter versus 4 points and 2 parameters can be found in Table 8.2.

Using both a cubic and linear fit gives us confidence that we are using a small enough magnetic field for our level of precision. Since the fit is constrained to go through the point $(0,0)$ we can get a magnetic moment value using only a single field strength. Without higher field strengths, however, it would be impossible to tell if there are higher order contributions in effect. If our lattice was half the size, for example, the smallest field strength available would have large $B^{3}$ contributions such that the measured magnetic moment value would be smaller. A second field strength is required to be able to see that there are higher order effects in play.

\subsubsection{Magnetic moment as a function of pion mass}

The magnetic moment is taken from the linear coefficients of the fits to the field. The magnetic moment is reported in units of nuclear magnetons, which are reached by,

$$
\mu=\frac{\Delta E}{e B}\left[\frac{e}{2 M_{N}}\right] 2 M_{N}
$$



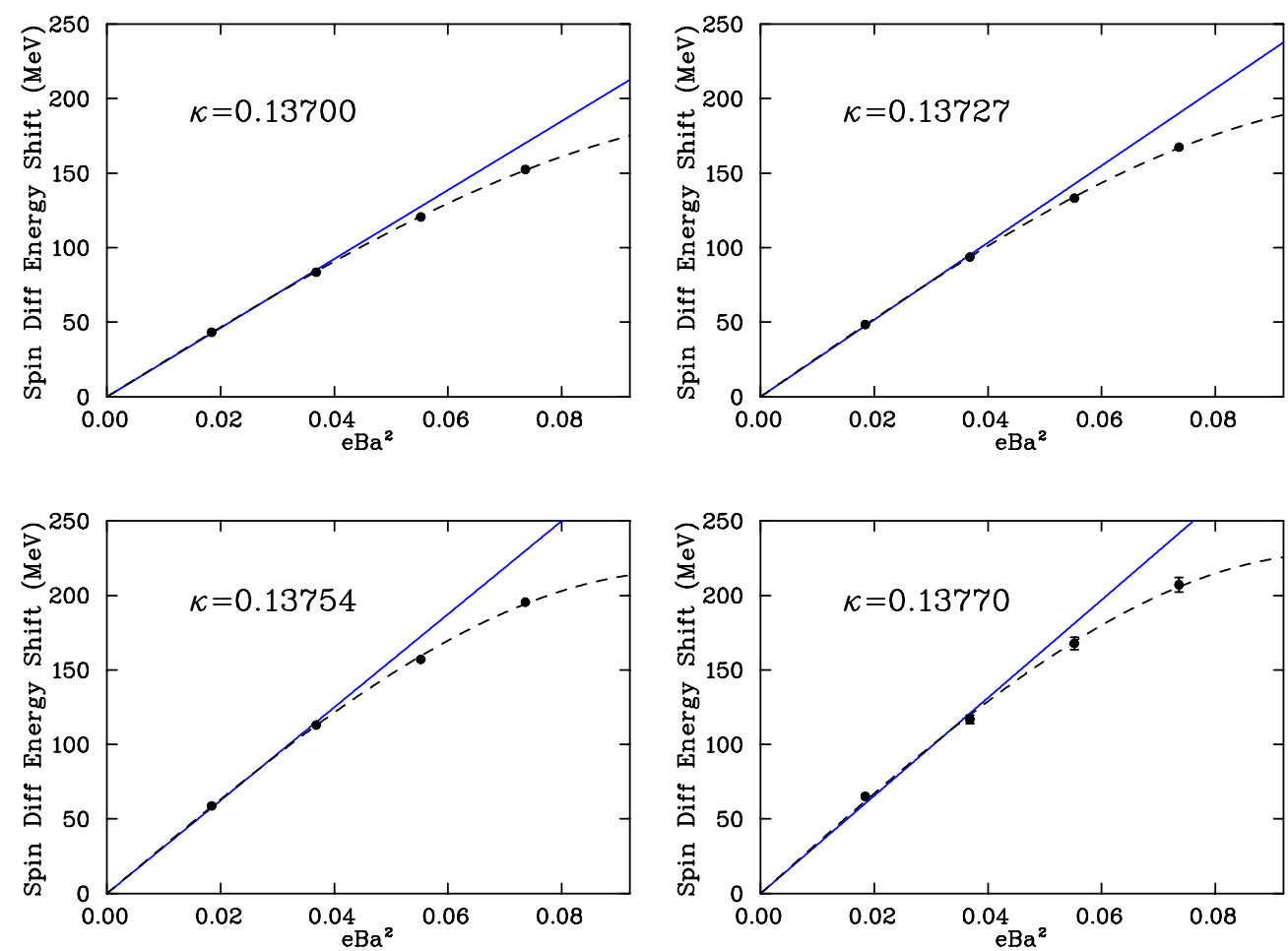

Figure 8.6: Plots of the fits of the spin-difference energy shift to the magnetic field strength for the neutron. The solid line is a one parameter linear fit to just the first two points. The dashed line is a two parameter cubic fit to all four points.

\begin{tabular}{c|cccc}
\hline \hline & \multicolumn{2}{|c}{2 point, 1 parameter } & \multicolumn{2}{c}{ 4 point, 2 parameter } \\
\hline$\kappa$ & $\mu_{n}\left(\mu_{N}\right)$ & $\chi_{\text {dof }}^{2}$ & $\mu_{n}\left(\mu_{N}\right)$ & $\chi_{\text {dof }}^{2}$ \\
\hline 0.13700 & $-1.17(2)$ & 12.2 & $-1.19(2)$ & 1.3 \\
0.13727 & $-1.28(2)$ & 4.6 & $-1.32(2)$ & 0.6 \\
0.13754 & $-1.43(3)$ & 4.4 & $-1.46(3)$ & 1.3 \\
0.13770 & $-1.43(3)$ & 6.8 & $-1.48(4)$ & 1.6 \\
\hline \hline
\end{tabular}

Table 8.2: Magnetic moment values and $\chi_{\text {dof }}^{2}$ values from the fits of the energy shift to the field strength for the neutron in units of nuclear magnetons $\left(\mu_{N}\right)$ at each $\kappa$ value comparing the 1 parameter 2 point fits with the 2 parameter 4 point fits. 

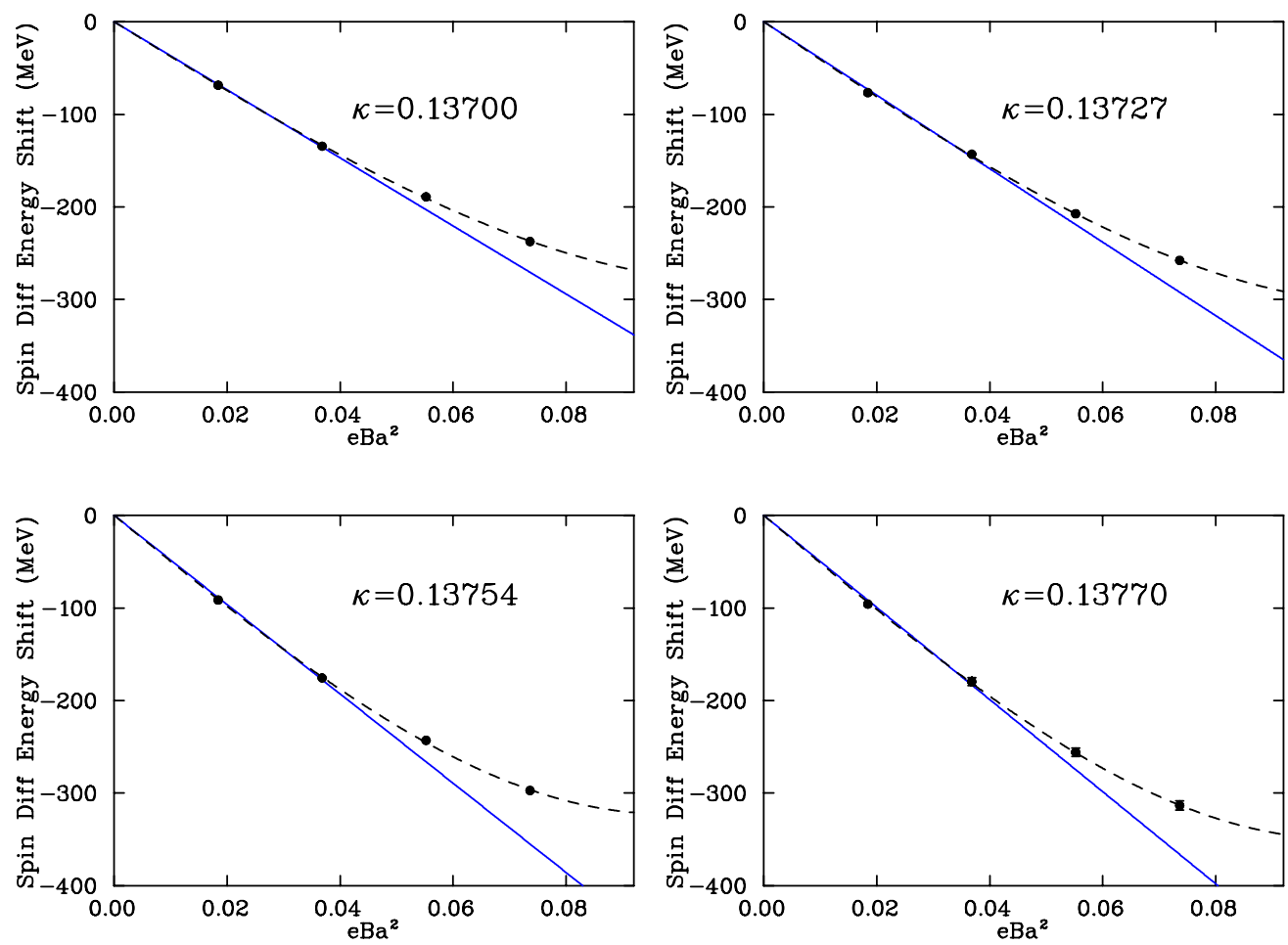

Figure 8.7: Plots of the fits of the spin-difference energy shift to the magnetic field strength for the proton. The solid line is a one parameter linear fit to just the first two points. The dashed line is a two parameter cubic fit to all four points. 
where we have started with Eq. (8.13) and introduced the elementary charge $e$ since we actually fit the energy shift against $e B$, such that $\Delta E / e B$ is equal to the fit parameter $a$ in Eq. (8.18). We then bring in twice the physical nucleon mass $M_{N}$ in order to get the nuclear magneton $\left(\mu_{N}=\frac{e \hbar}{2 M_{N}}\right)$, noting that we are using natural units $(c=\hbar=1)$.

Figure 8.8 displays a plot of the proton magnetic moment values as a function of the square of the pion mass as a measure of the quark mass. It includes both the dynamical results, the earlier quenched results and a comparison to a three point function calculation [32]. The details of the quenched calculation can be found in Appendix A. There is decent agreement between the three sets of results, with the dynamical values sitting on a slightly higher curve for the three heavier quark masses where statistical errors are small enough to discern a difference. This is a reasonable behaviour to expect as arising from the addition of sea quark contributions [35].

Figure 8.9 shows the neutron magnetic moment as a function of the pion mass squared. Again we see reasonable agreement between the quenched, dynamical and three point function results, with the heavier dynamical points seeming to trend to a larger value than the quenched. We see the lightest dynamical point has a smaller value than expected with the trend approximately flat from the second lightest mass. A similar effect is observed for the proton. Seeing both the proton and neutron have the same trend suggests that there is some systematic effect in play. The effect is mostly likely due to finite volume effects, which would be expected to increase significantly as we reach lighter quark masses [36]. This is also consistent with the fact that the quenched results are from a larger physical lattice than the dynamical results.

The lines are chiral fits using the approach from Ref. [37], and guide the anticipated trajectory to the physical point. The reason the extrapolated values are smaller in magnitude than the experimental values is expected to come from finite volume effects at all points as those have not been examined here.

\subsection{Odd Parity Nucleon}

In addition to the ground state nucleon we are also interested in the magnetic moment of excited states of the proton and neutron, including odd-parity states. These can also be calculated using the background field method. 


\begin{tabular}{c|cccccc}
\hline \hline$\kappa$ & 0.13700 & 0.13727 & 0.13754 & 0.13770 & extrap. & exp. \\
\hline$\mu_{p}\left(\mu_{N}\right)$ & $1.86(2)$ & $2.01(3)$ & $2.23(3)$ & $2.28(6)$ & $2.69(12)$ & 2.79 \\
$\mu_{n}\left(\mu_{N}\right)$ & $-1.19(1)$ & $-1.32(2)$ & $-1.46(2)$ & $-1.48(3)$ & $-1.83(8)$ & -1.91 \\
\hline \hline
\end{tabular}

Table 8.3: Magnetic moment values for the proton and neutron at each quark mass as well as the extrapolated and experimental values. Values are in units of nuclear magnetons.

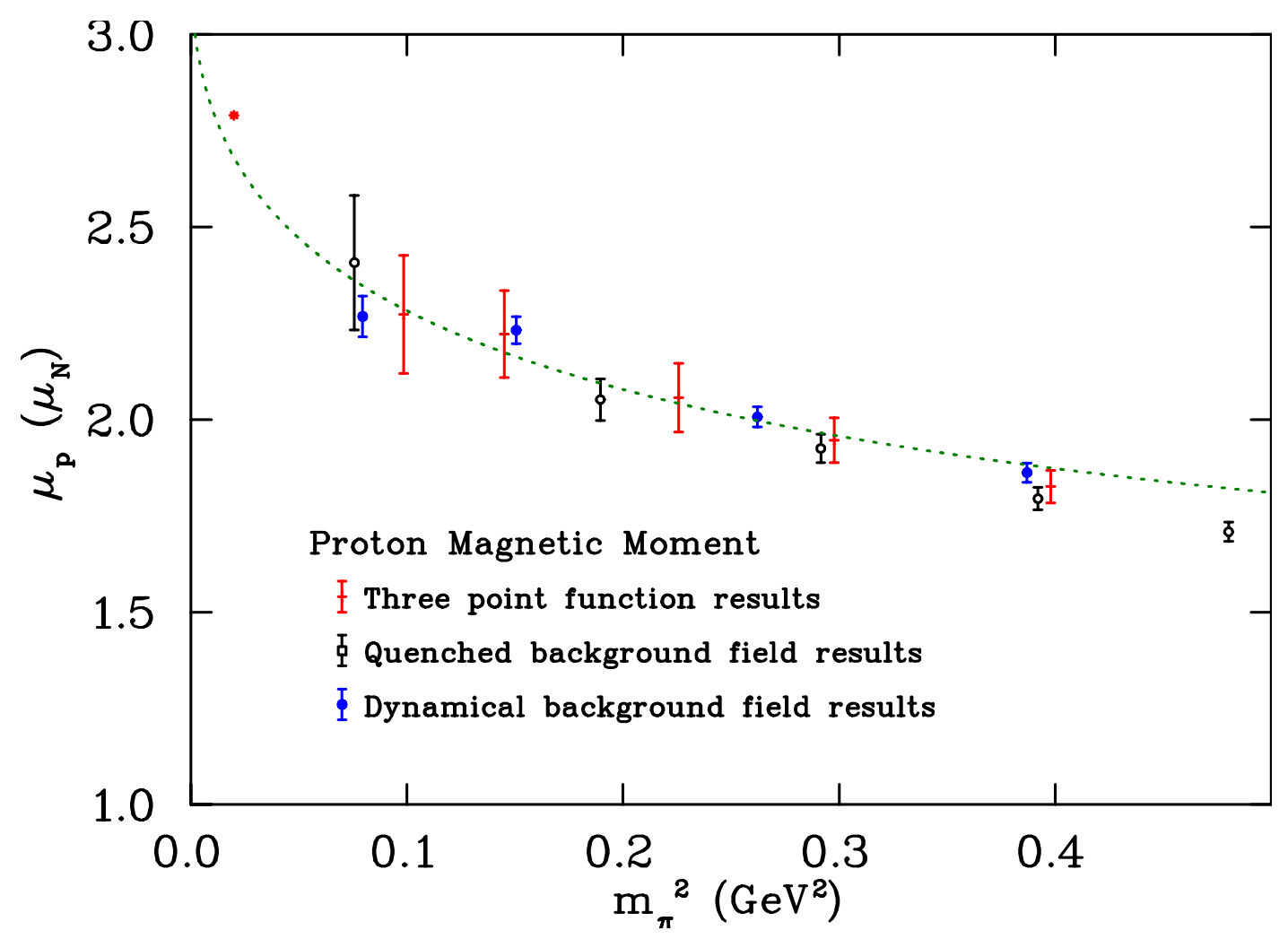

Figure 8.8: The magnetic moment of the proton as a function of pion mass squared. The left-most point is the experimental value. The dotted line is a chiral fit based on Ref. [37]. The three point function results are from Ref. [32]. 


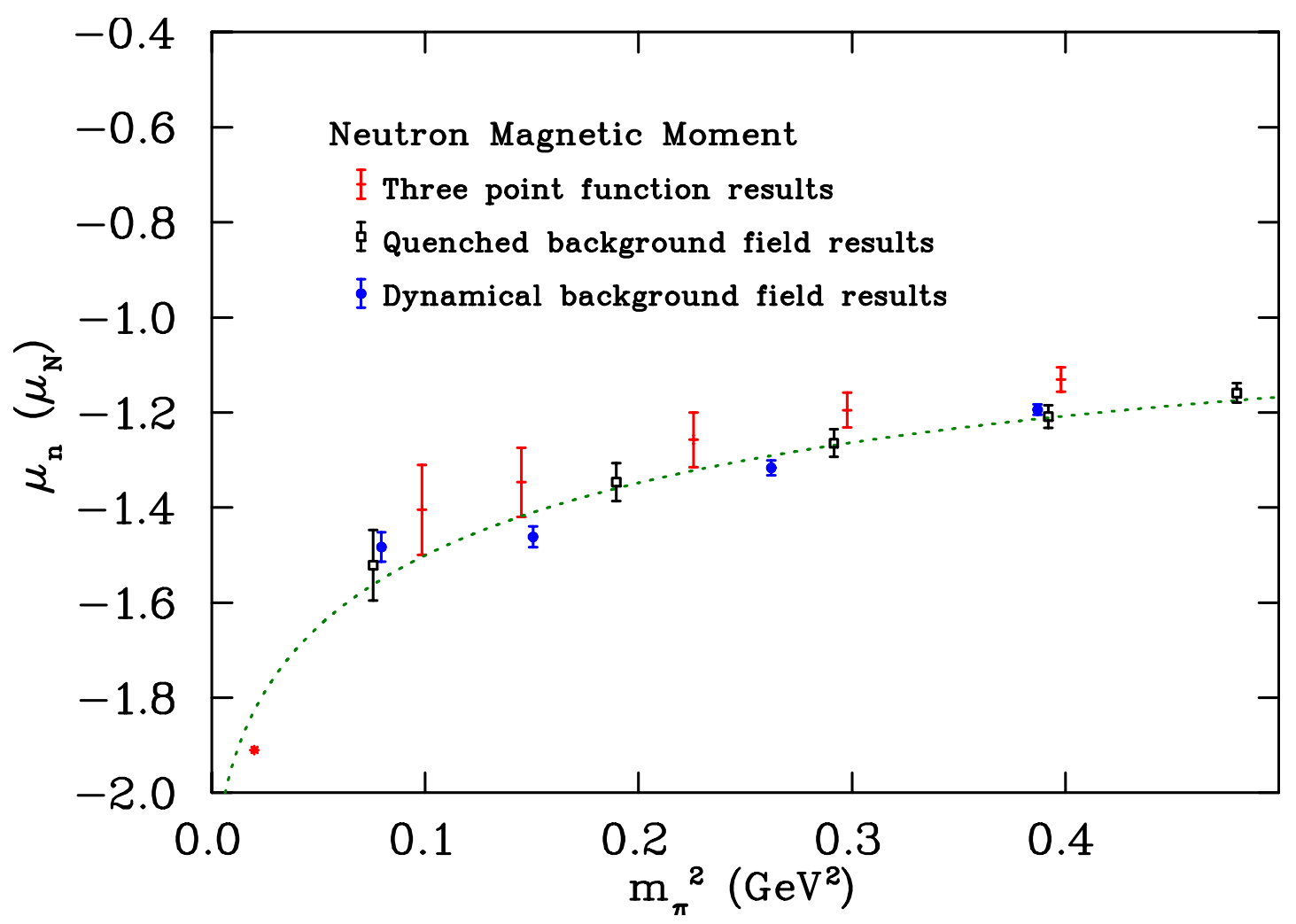

Figure 8.9: The magnetic moment of the neutron as a function of pion mass squared. The left-most point is the experimental value. The dotted line is a chiral fit based on Ref. [37]. The three point function results are from Ref. [32]. 
Only one such calculation has been attempted before [38], leaving a lot of potential for discovery.

We can access odd-parity states from our normal correlation functions that use interpolators which transform positively under parity transformations using a parity projection. The Euclidean space correlation function can be written as,

$$
G(t, \mathbf{p})=\sum_{B, s} e^{-E_{B} t}\langle\Omega|\chi(0)| B, \mathbf{p}, s\rangle\langle B, \mathbf{p}, s|\bar{\chi}(0)| \Omega\rangle,
$$

Where $\Omega$ is the QCD vacuum, $\chi$ and $\bar{\chi}$ are the sink and source interpolating fields, $B$ are intermediate baryon states, $\mathbf{p}$ is the momentum of the state and $s$ is the spin. We define the coupling of each state to the interpolators $\lambda, \bar{\lambda}$ and the Dirac spinors $u, \bar{u}$. For even-parity we have, at the sink,

$$
\left\langle\Omega|\chi| B^{+}, \mathbf{p}, s\right\rangle=\lambda_{B^{+}} \sqrt{\frac{M_{B^{+}}}{E_{B^{+}}}} u_{B^{+}}(p, s),
$$

which for odd-parity is replaced by,

$$
\left\langle\Omega|\chi| B^{-}, \mathbf{p}, s\right\rangle=\lambda_{B^{-}} \sqrt{\frac{M_{B^{-}}}{E_{B^{-}}}} \gamma_{5} u_{B^{-}}(p, s) .
$$

For odd-parity at the source we get,

$$
\begin{aligned}
\left\langle B^{-}, \mathbf{p}, s|\bar{\chi}| \Omega\right\rangle & =\bar{\lambda}_{B^{-}} \sqrt{\frac{M_{B^{-}}}{E_{B^{-}}}} u_{B^{-}}^{\dagger}(p, s) \gamma_{5} \gamma_{0} \\
& =-\bar{\lambda}_{B^{-}} \sqrt{\frac{M_{B^{-}}}{E_{B^{-}}}} \bar{u}_{B^{-}}(p, s) \gamma_{5}
\end{aligned}
$$

So we then have, for odd-parity,

$$
\begin{aligned}
& \sum_{B^{-}, s}\left\langle\Omega|\chi| B^{-}, \mathbf{p}, s\right\rangle\left\langle\Omega|\chi| B^{-}, \mathbf{p}, s\right\rangle \\
& =\sum_{B^{-}}-\lambda_{B^{-}} \bar{\lambda}_{B^{-}} \gamma_{5} \sum_{s} u_{B^{-}}(\mathbf{p}, s) \bar{u}_{B^{-}}(\mathbf{p}, s) \gamma_{5} \\
& =\sum_{B^{-}}-\lambda_{B^{-}} \bar{\lambda}_{B^{-}} \gamma_{5} \frac{\gamma \cdot p+M_{B^{-}}}{2 E_{B^{-}}} \gamma_{5} \\
& =\sum_{B^{-}} \lambda_{B^{-}} \bar{\lambda}_{B^{-}} \frac{\gamma \cdot p-M_{B^{-}}}{2 E_{B^{-}}}
\end{aligned}
$$


which allows us to write our correlation function in terms of even and oddparity components,

$$
\begin{aligned}
G(t, \mathbf{p}) & =\sum_{B^{+}} \lambda_{B^{+}} \bar{\lambda}_{B^{+}} e^{-E_{B^{+}}} \frac{\gamma \cdot p+M_{B^{+}}}{2 E_{B^{+}}} \\
& -\sum_{B^{-}} \lambda_{B^{-}} \bar{\lambda}_{B^{-}} e^{-E_{B^{-}}} \frac{-\gamma \cdot p+M_{B^{-}}}{2 E_{B^{-}}} .
\end{aligned}
$$

At $\mathbf{p}=0$ we can define a parity projection operator,

$$
\Gamma^{ \pm}=\frac{1}{2}\left(\gamma_{0} \pm 1\right),
$$

which allows us to isolate the odd-parity parts of the correlation function, as they are in the $(3,3)$ and $(4,4)$ components of the Dirac matrix for spinup and spin-down respectively. This gives us correlation functions which asymptote to the lowest lying odd-parity state. We can take the difference of spins and fit the energy shift to extract the magnetic moment just as in the ground state case. Statistical uncertainties will prevent us from exploring the polarisabilities of these states.

\subsubsection{Initial results}

Initially we tried the same interpolating field as we used for the ground state calculation,

$$
\chi_{1}=\left(d^{T} C \gamma_{5} u\right) d,
$$

which has historically proved effective at accessing the nucleon ground state. This gave us very poor signal when looking at the odd-parity spin-difference energy shift. We therefore decided to try using another nucleon interpolating field,

$$
\chi_{2}=\left(d^{T} C u\right) \gamma_{5} d .
$$

This interpolating field resulted in a strong signal for the spin-difference for both the proton and the neutron, as shown in Figures 8.10 and 8.11. These effective energies display the characteristic linear progression we expect from a magnetic moment effect. Although these energy shifts could easily be fit to extract a magnetic moment value we needed to understand why we got such different results with $\chi_{1}$ and $\chi_{2}$. 


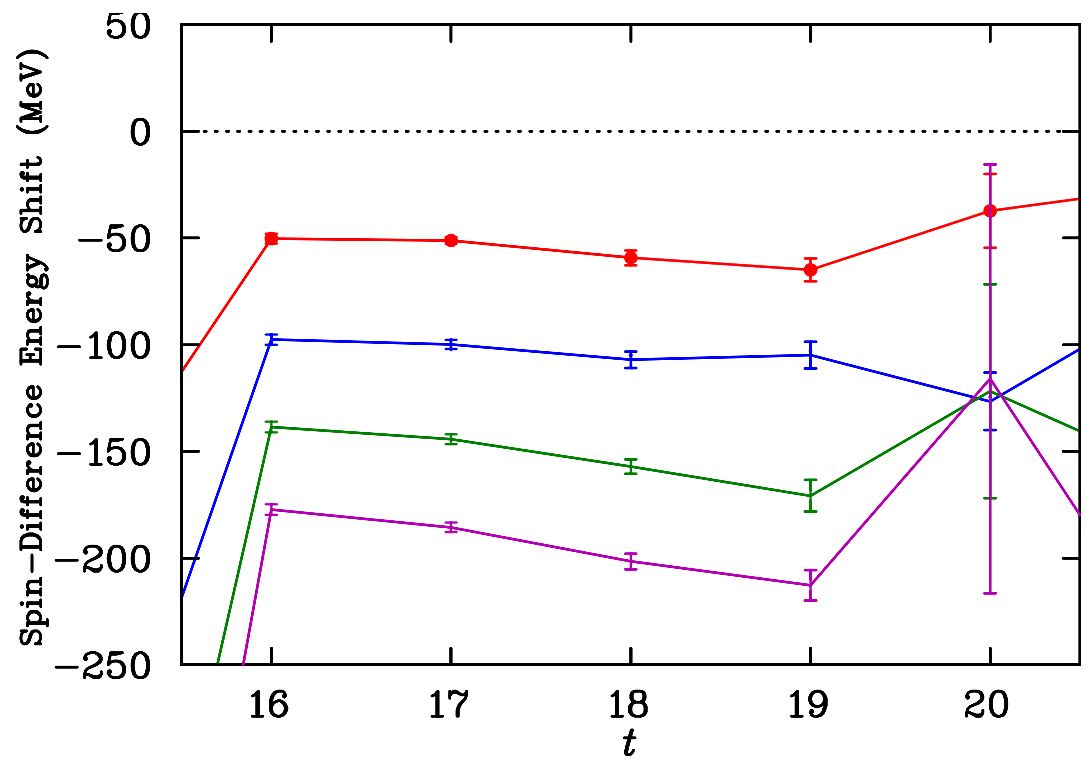

Figure 8.10: Spin-difference energy shift for the odd-parity proton using a $\chi_{2}$ interpolating field at $\kappa=0.13700$ for all four field strengths.

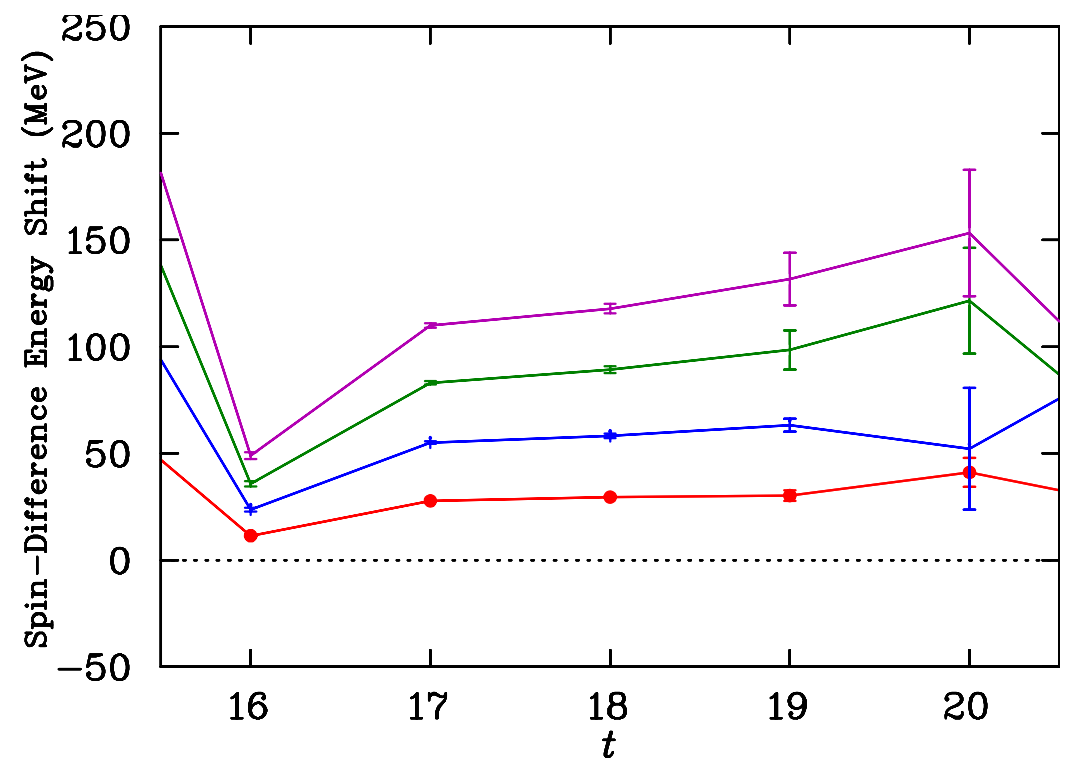

Figure 8.11: Spin-difference energy shift for the odd-parity neutron using a $\chi_{2}$ interpolating field at $\kappa=0.13700$ for all four field strengths. 
The reason for the difference in results for the two interpolator forms is that the two lowest lying $-1 / 2$ states, the $N^{*}(1535)$ and the $N^{*}(1650)$, are quite close to each other in mass. The difference is just about $100 \mathrm{MeV}$, compared to $500 \mathrm{MeV}$ between the ground state and the first even-parity excitation (the Roper resonance). As a result the lowest lying odd-parity state is not easily isolated by just waiting for the next state to be exponentially suppressed. This means that our $\chi_{1}$ and $\chi_{2}$ energy shifts contain contributions from both of the two lowest lying odd-parity states. The coupling of each of these states is different for each interpolating field, giving us the difference in the effective energies. Any result we get for the moment using $\chi_{2}$ could not be said to have come from a well defined state. We need to effectively isolate the two states, which we do using the variational method.

\subsubsection{Variational method}

Accessing the masses of ground states requires only the exponential suppression of excited states at large Euclidean times. In order to access excited state masses we use the variational method [39-43]. We construct a correlation matrix from a set of basis operators which spans the state space. Using $N$ different interpolators we can isolate up to $N$ different states from the spectrum. We write the correlation function matrix at zero momentum as a sum over all excited states,

$$
\begin{aligned}
G_{i j}(t) & =\sum_{\mathbf{x}}\left\langle\Omega\left|\chi_{i}(x) \bar{\chi}_{j}(0)\right| \Omega\right\rangle \\
& =\sum_{\alpha} \lambda_{i}^{\alpha} \bar{\lambda}_{j}^{\alpha} e^{-M_{\alpha} t},
\end{aligned}
$$

where $\lambda_{i}$ and $\bar{\lambda}_{j}$ are the couplings of state $\alpha$ to the interpolating fields $\chi_{i}$ and $\bar{\chi}_{j}$ at the sink and source respectively. We use our operator basis to construct a linear combination of interpolators which perfectly isolates each of $N$ baryon states $\left|B_{\alpha}\right\rangle$,

$$
\begin{aligned}
\bar{\phi}^{\alpha}(x) & =\sum_{j} u_{j}^{\alpha} \bar{\chi}_{j}(x) \\
\phi^{\alpha}(x) & =\sum_{i} v_{i}^{\alpha} \chi_{i}(x),
\end{aligned}
$$

such that $\phi^{\alpha}$ and $\bar{\phi}^{\alpha}$ have good overlap with state $\alpha$ and zero overlap with any other state. 
Since the only $t$ dependence in Eq. (8.31) comes from the exponential term we can write a recurrence relation for times $t_{0}$ and $\Delta t$ using the vector from Eq. (8.32),

$$
G_{i j}\left(t_{0}+\Delta t\right) u_{j}^{\alpha}=e^{-m_{\alpha} \Delta t} G_{i j}\left(t_{0}\right) u_{j}^{\alpha},
$$

for sufficiently large $t_{0}$ and $\Delta t$. We can then multiply by $\left[G_{i j}\left(t_{0}\right)\right]^{-1}$ from the left to get,

$$
\left[\left(G\left(t_{0}\right)\right)^{-1} G\left(t_{0}+\Delta t\right)\right] u^{\alpha}=e^{-m_{\alpha} \Delta t} u^{\alpha}
$$

This is an eigenvalue equation for eigenvector $u^{\alpha}$ with eigenvalue $c^{\alpha}=\exp \left(-m_{\alpha} \Delta t\right)$. We can do the same thing using the sink eigenvector $v^{\alpha}$ on the left,

$$
v_{i}^{\alpha} G_{i j}\left(t_{0}+\Delta t\right)=e^{-m_{\alpha} \Delta t} v_{i}^{\alpha} G_{i j}\left(t_{0}\right),
$$

and then multiplying by $\left[G_{i j}\left(t_{0}\right)\right]^{-1}$ from the right,

$$
v^{\alpha}\left[G\left(t_{0}+\Delta t\right)\left(G\left(t_{0}\right)\right)^{-1}\right]=e^{-m_{\alpha} \Delta t} v^{\alpha}
$$

These two eigenvalue equations are solved and the eigenvectors are then used to diagonalise the correlation matrix at time $t_{0}$ and $t_{0}+\Delta t$, projecting out the mass eigenstates,

$$
v_{i}^{\alpha} G_{i j}(t) u_{j}^{\beta}=\delta^{\alpha \beta} \lambda^{\alpha} \bar{\lambda}^{\beta} e^{-m_{\alpha} t} .
$$

There is some freedom to the choice of the variational parameters $t_{0}$ and $\Delta t$. The $t_{0}$ must be early enough that there is enough of all the states we want to project still present, but not so early that a large amount of higher unwanted excited states are contributing significantly. We tried out a number of values relative to the source at $t_{s}=16$, with $t_{0}=17,18,19,20$ and $\Delta t=1,2,3,4$. We found the best results came from starts of 18 and 19 and steps of 2 and 3 , which seems to be fairly typical [39]. Values in the results section come from $t_{0}=18$ and $\Delta t=2$.

Using the parity and eigenstate projected correlation functions we can use our standard analysis techniques to extract effective masses. The same techniques can be applied to background field correlation functions, only with a few subtleties. A separate set of eigenvalue equations must be solved for each combination of spin-up and spin-down, field on and field off. The point is that the Hilbert space changes and one must first isolate the state before combining it with states from the other Hilbert spaces. In other words, the eigenvectors $u_{i}^{\alpha}$ and $v_{j}^{\beta}$ are field and spin dependent and a recurrence relation cannot be written for combinations of spins and fields. 


\subsubsection{Results}

We performed our analysis using a $2 \times 2$ correlation matrix with $\chi_{1}$ and $\chi_{2}$ type interpolators with 100 sweeps of Gaussian smearing at the source and a point sink. An important concern when using the variational method with background field method calculations is correctly identifying the states that are given by the analysis. The eigenvector equation is solved separately for each spin and field strength and for each jackknife sub-ensemble in the complement set before the ratio in Eq. (8.17) is taken, so we must ensure that for each case we are combining the same state for field on and field off and for spin-up and spin-down.

The eigenmasses that result from solving the eigenvalue equation come in no particular order. The easiest way to order them is by the magnitude of the mass, which works well in a well spaced spectrum, but fails in our case. We found that for both the proton and neutron excited states we had one state which increased in energy when the field was turned on and one state which decreased with the field on. This indicates that one has a positive magnetic moment and the other has a negative moment. Since the direction of the energy shift is opposite for opposite spins, this means that for either spin-up or spin-down the field will move the effective energies of each state towards each other, potentially crossing. The difference in the zero-field masses of the states was small enough that we found even at the smallest field strength the energies were degenerate for one of the spins and that at higher field strengths the order switched. Therefore we had to find another method for ordering the states.

This is similar to the problem of tracking states across multiple pion masses when using the variational method for pure spectroscopy, as the ordering of the masses may change. One technique is to create a matrix from the products of the eigenvectors at two different quark masses [42], or in our case, field strengths,

$$
M^{\alpha \beta}=\mathbf{w}^{\alpha}(B) \cdot \mathbf{w}^{\beta}\left(B^{\prime}\right) .
$$

Here the state labels $\alpha$ and $\beta$ vary across row and column respectively and $w^{\alpha}=G^{\frac{1}{2}}\left(t_{o}\right) u^{\alpha}$ is an orthogonal version of the eigenvector obtained directly from the symmetric eigenvalue equation. We begin with Eq. (8.34) and

multiply by $G^{-\frac{1}{2}}\left(t_{0}\right)$ from the left and insert the identity $I=G^{-\frac{1}{2}}\left(t_{0}\right) G^{\frac{1}{2}}\left(t_{0}\right)$ 
to get,

$$
\begin{aligned}
G^{-\frac{1}{2}}\left(t_{0}\right) G\left(t_{0}+\Delta t\right) G^{-\frac{1}{2}}\left(t_{0}\right) G^{\frac{1}{2}}\left(t_{0}\right) u^{\alpha} & =e^{-m_{\alpha} \Delta t} G^{\frac{1}{2}}\left(t_{0}\right) u^{\alpha} \\
G^{-\frac{1}{2}}\left(t_{0}\right) G\left(t_{0}+\Delta t\right) G^{-\frac{1}{2}}\left(t_{0}\right) w^{\alpha} & =e^{-m_{\alpha} \Delta t} w^{\alpha}
\end{aligned}
$$

such that $G^{-\frac{1}{2}}\left(t_{0}\right) G\left(t_{0}+\Delta t\right) G^{-\frac{1}{2}}\left(t_{0}\right)$ is a real symmetric matrix with orthogonal eigenvectors $w^{\alpha}$. The values of the matrix elements can be used to determine the order of states. Consider a case where the state with the lower eigenvalue is labelled $a$ and the higher eigenvalue state is labelled $b$. When $M^{a a}$ and $M^{b b}$ are large $(\sim 1)$ it means that the states are coupling strongly to the same source/sink at both field strengths, so the labelling of those states is correct. When $M^{a b}$ and $M^{b a}$ are larger it means that those two eigenstates have swapped positions in the spectrum and the labels need to be swapped.

We found that this technique was partially effective, but that for some field changes the diagonal term was only slightly larger than the off-diagonal. In other cases one of the diagonal terms was the largest, but the off-diagonals were larger than the other diagonal term, making the order ambiguous. This led to getting the correct state labels for some sub-ensembles but not others, especially at the first non-zero field strength, which revealed the error in a massive uncertainty in the eigenvalues at that field strength.

We noted that for both the odd-parity proton and neutron the first state always had a large $\chi_{2}$ component and the second state had a large $\chi_{1}$ component, while the smaller component fluctuated quite a bit. Therefore we chose to order the states simply by finding single largest contribution in $u^{\alpha}$ or $v^{\alpha}$ to either state. If the largest contribution was from the $\chi_{2}$ interpolating field then that state was designated state 1 and the other state was state 2 and vice versa if $\chi_{1}$ was the largest component. This technique was very effective and gave us unambiguous states from which to construct correlation function ratios.

Figure 8.12 shows the left eigenvector values for the odd-parity proton and neutron. In each case there is a clear dominant contribution from either $\chi_{1}$ or $\chi_{2}$. A reasonably systematic variation of the eigenvectors is observed as the external field changes the Hilbert space. The largest errors are generally found on the zero field and smallest two fields. This is in contrast to what we have seen in effective energies, where the errors generally increase with the field strength. 


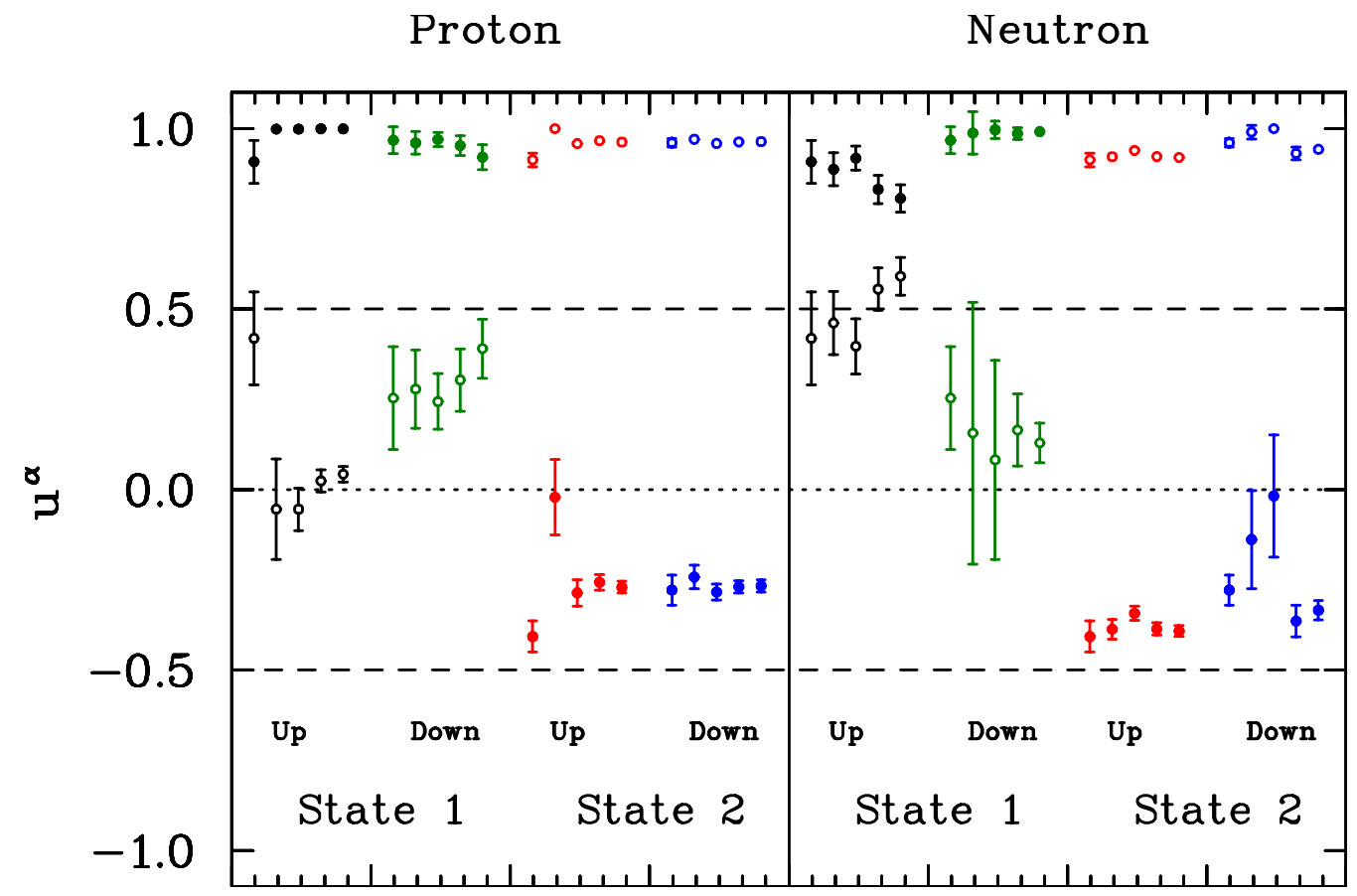

Figure 8.12: Plot of the left eigenvector values associated with the point sink for the odd-parity proton and neutron at each spin and field strength for the heaviest pion mass. Each group of points goes from left to right, zero field to largest magnetic field. The hollow circles are for $\chi_{1}$ and the solid circles are for $\chi_{2}$. 


\subsubsection{Effective energies}

Once we have a way to keep our ordering of states consistent for changing fields and masses we still need to identify what those states actually are. Figure 8.13 shows the spin-down effective mass at zero field for state 1 and 2. The masses begin to overlap and cross in the region where the signal is becoming noisy, but at the important early region where the eigenvalue equation was actually solved and the projected mass can be fit there is a clear distinction between the masses. The ordering of states at non-physical pion mass is non-trivial as the ordering can change [42,43], but our result is consistent with previous odd-parity mass spectrum calculations where a lighter quark mass next to the physical values has been investigated. We therefore determine that state 1 is the $S_{11}(1535)$ and state 2 is $S_{11}(1650)$.

Figures 8.14 to 8.17 display the projected spin-difference energy shifts for the odd-parity proton. The first state has negative energy shifts which are reasonably flat and display the characteristic linear progression with increasing field strength. The plateaus are lost to noise much earlier than the even-parity ones, but are not problematic to fit. Most of the curves are flat right from time slice 17, however some plateau later so to avoid any small excited contributions still present at the earliest times we choose for our fit windows 20-21 at $\kappa=0.13700$ and 19-21 at the other masses. These fit windows give $\chi_{\text {dof }}^{2}$ values close to 1 except at the lightest mass considered where a couple of them are a bit higher due to there being no ideal fit window. At $\kappa=0.13754$ the second field strength sits much closer to the third field strength than we would expect.

Looking at the second state the curves are much less clear. There are still significant excited state contributions at the first few time slices, but we expect the third and higher odd-parity states to be high enough in energy to be exponentially suppressed after a short time. However if one of these higher states had a large magnetic moment it could bring the energy down enough to prevent it being suppressed. The second state signal is lost to noise earlier than the first, giving us little time in which to find a plateau. Some fields have fittable plateaus from 18-19, but enough of them are still trending larger at these times to make us believe that this is too early. We again use $\chi_{\text {dof }}^{2}$ values to guide our fit windows. At $\kappa=0.13700$ the best window is $20-21$, which gives a $\chi_{\text {dof }}^{2}$ less than one for each field strength. At the other masses we use a window of 19-20. This gives a slightly too large

$\chi_{\text {dof }}^{2}$ value $(\approx 3)$ at the second field strength for $\kappa=0.13754$ and the third 

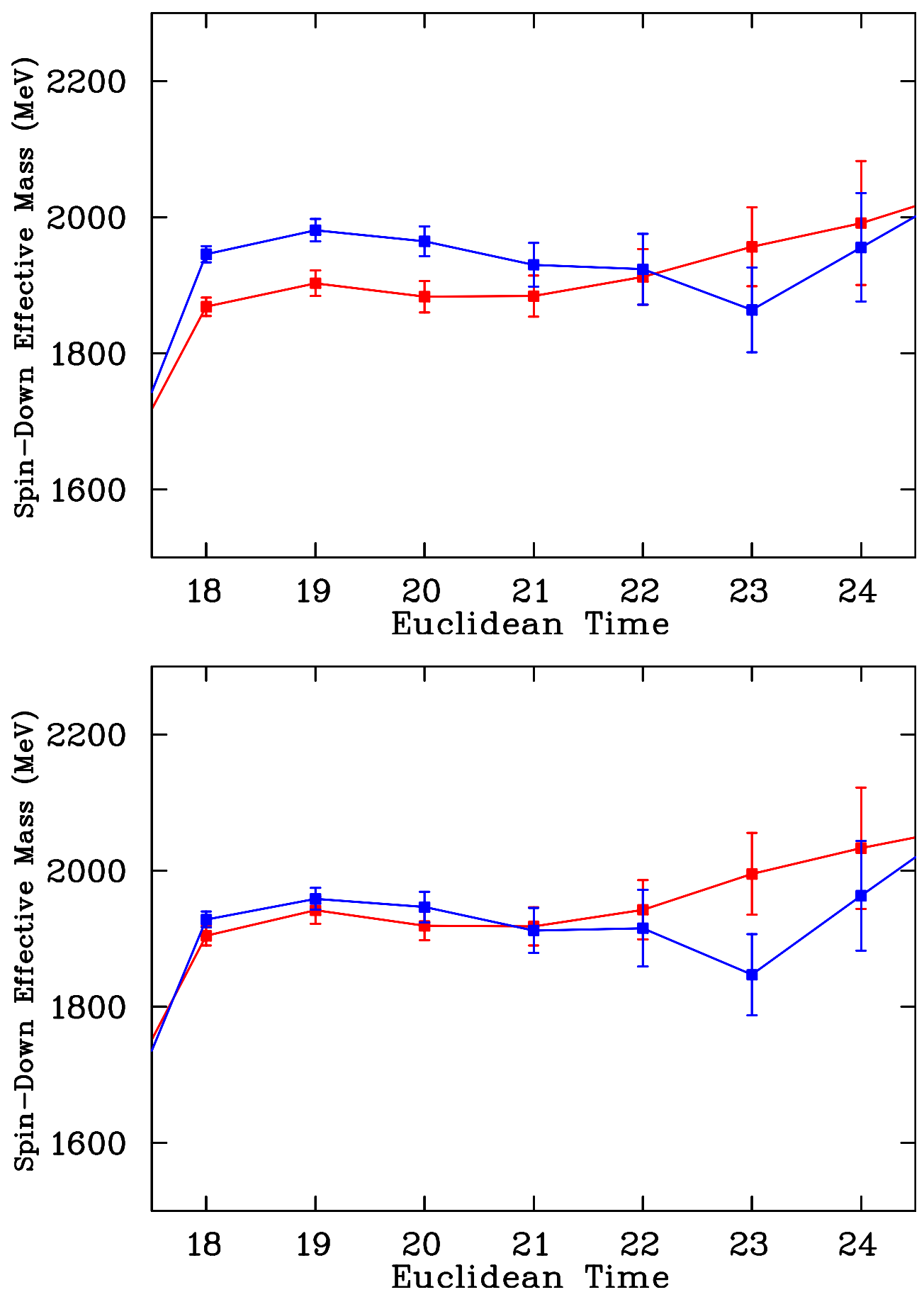

Figure 8.13: Spin-down effective mass plots for states 1 and 2 from projected correlation functions at $\kappa=0.13700$. Top plot is for zero background field and below is for the smallest non-zero field strength used. 


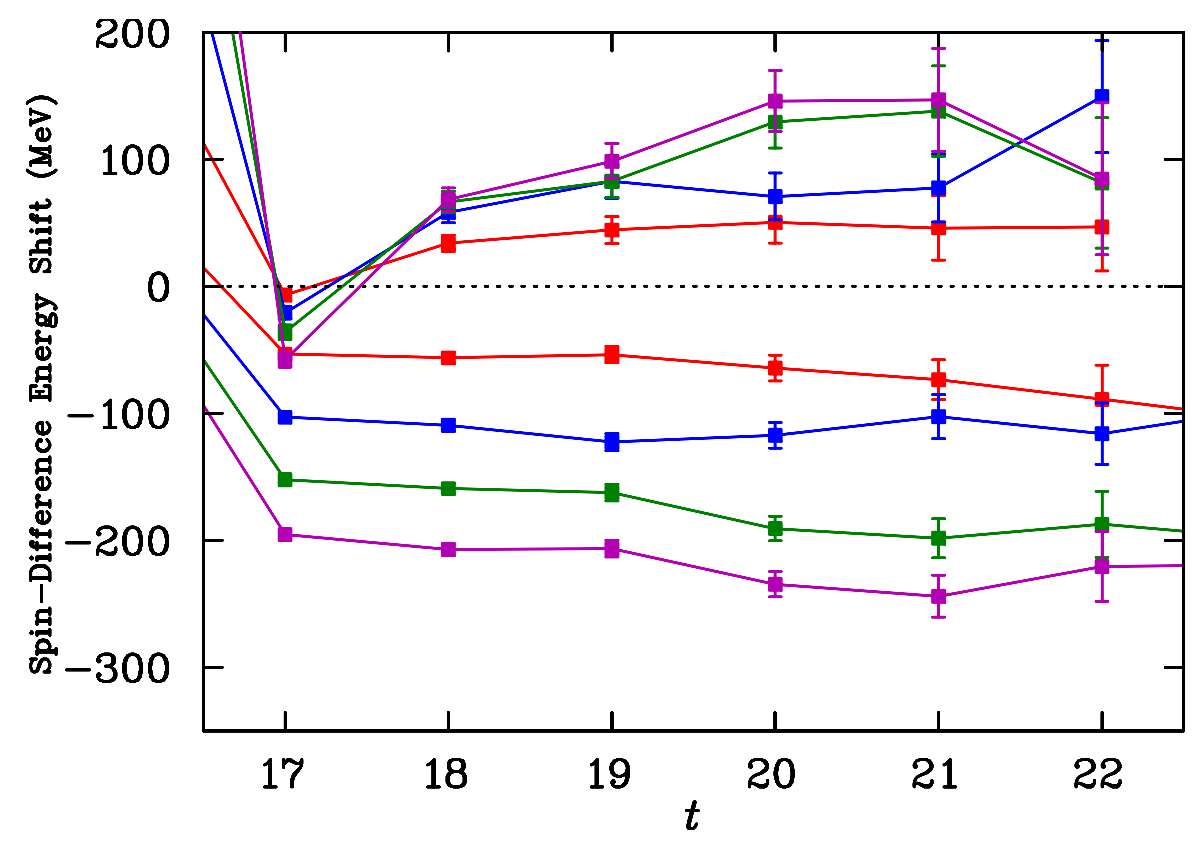

Figure 8.14: Spin-difference energy shift plot for odd-parity proton states from the variational analysis at $\kappa=0.13700$. Two states are illustrated for four different field strengths. State 1 is the negative energy shifts and state 2 is the positive shifts. The curves are ordered with the magnitude of the shifts increasing with the strength of the magnetic field as of times 20-21.

field strength for $\kappa=0.13770$, but the other field strengths give very good values, making this the best window overall. At $\kappa=0.13754$ the second field strength actually sits above the fourth, which is completely at odds with our expectations. There is no fit window for which this isn't the case. Since this is the same mass where state 1 gave an unexpected shift for the second field we conclude that the variational analysis has had difficulty in separating the states at this field and mass. The second field for state two also sits higher than expected at the other masses as well.

Figures 8.18 to 8.21 display the projected spin-difference energy shifts for the odd-parity neutron. These follow the same general trends as the proton, but with smaller shifts and opposite signs. We use the same fit windows as for the proton, 19-21 for state one and 19-20 for state two, except at the heaviest mass considered, where both states are fit starting 1 time slice later. These fit windows give the $\chi_{\text {dof }}^{2}$ values closest to one. We have the same 


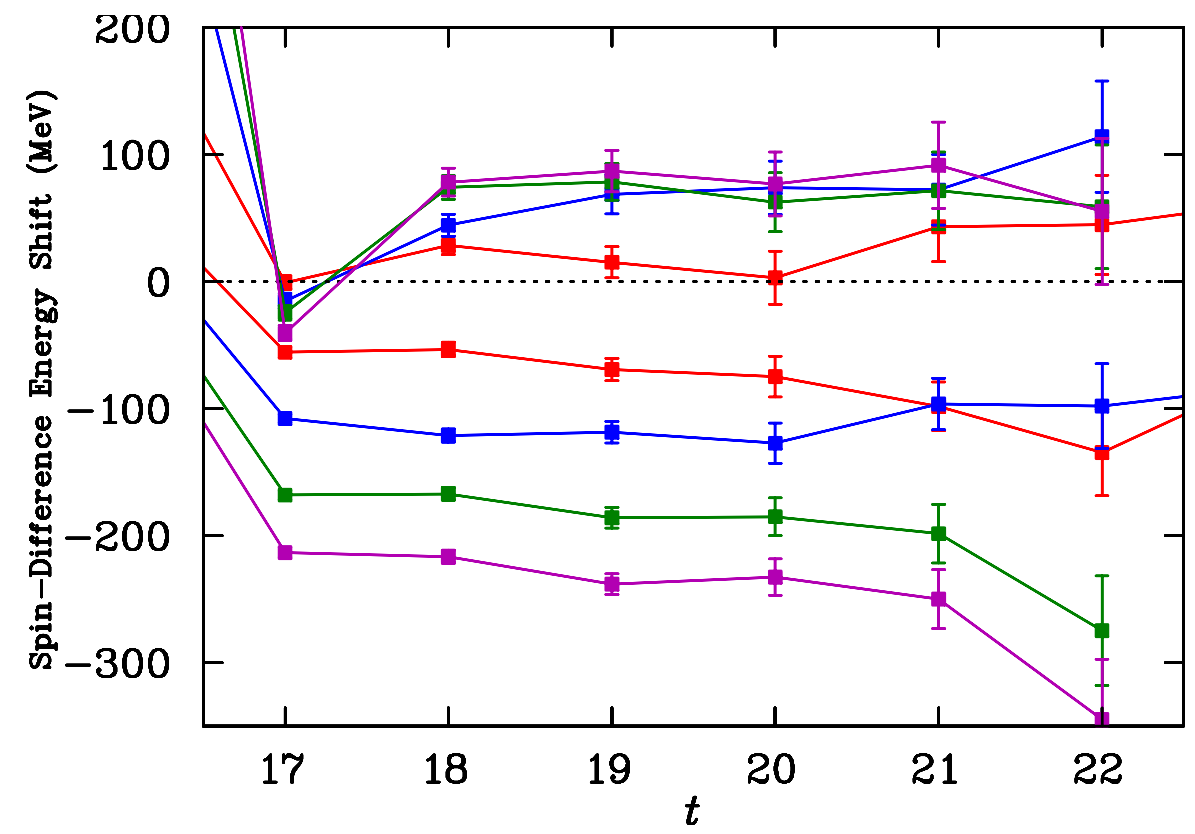

Figure 8.15: Spin-difference energy shift plot for odd-parity proton states from the variational analysis at $\kappa=0.13727$. Two states are illustrated for four different field strengths. State 1 is the negative energy shifts and state 2 is the positive shifts. The curves are ordered with the magnitude of the shifts increasing with the strength of the magnetic field as of times 18-19. 


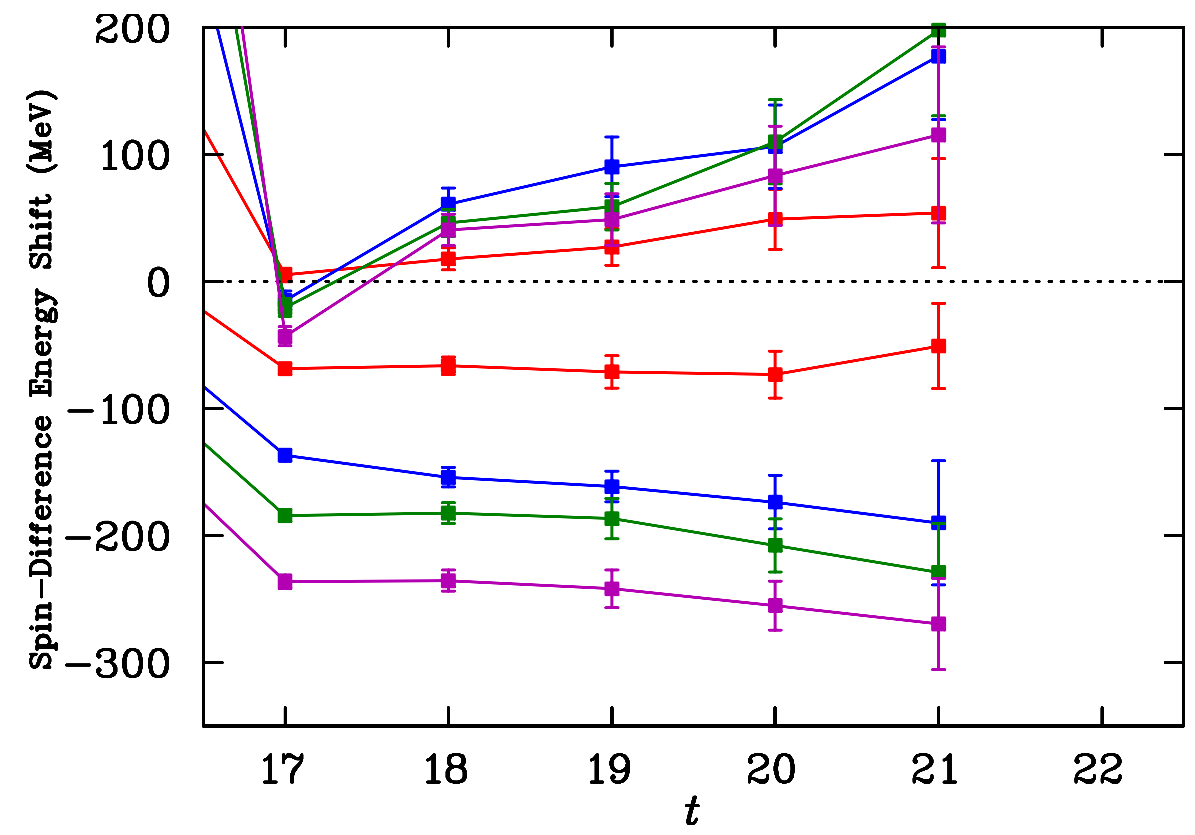

Figure 8.16: Spin-difference energy shift plot for odd-parity proton states from the variational analysis at $\kappa=0.13754$. Two states are illustrated for four different field strengths. State 1 is the negative energy shifts and state 2 is the positive shifts. The curves are ordered with the magnitude of the shifts increasing with the strength of the magnetic field except that for state 2 the second non-zero field strength gives the largest shift as of times 18-19. 


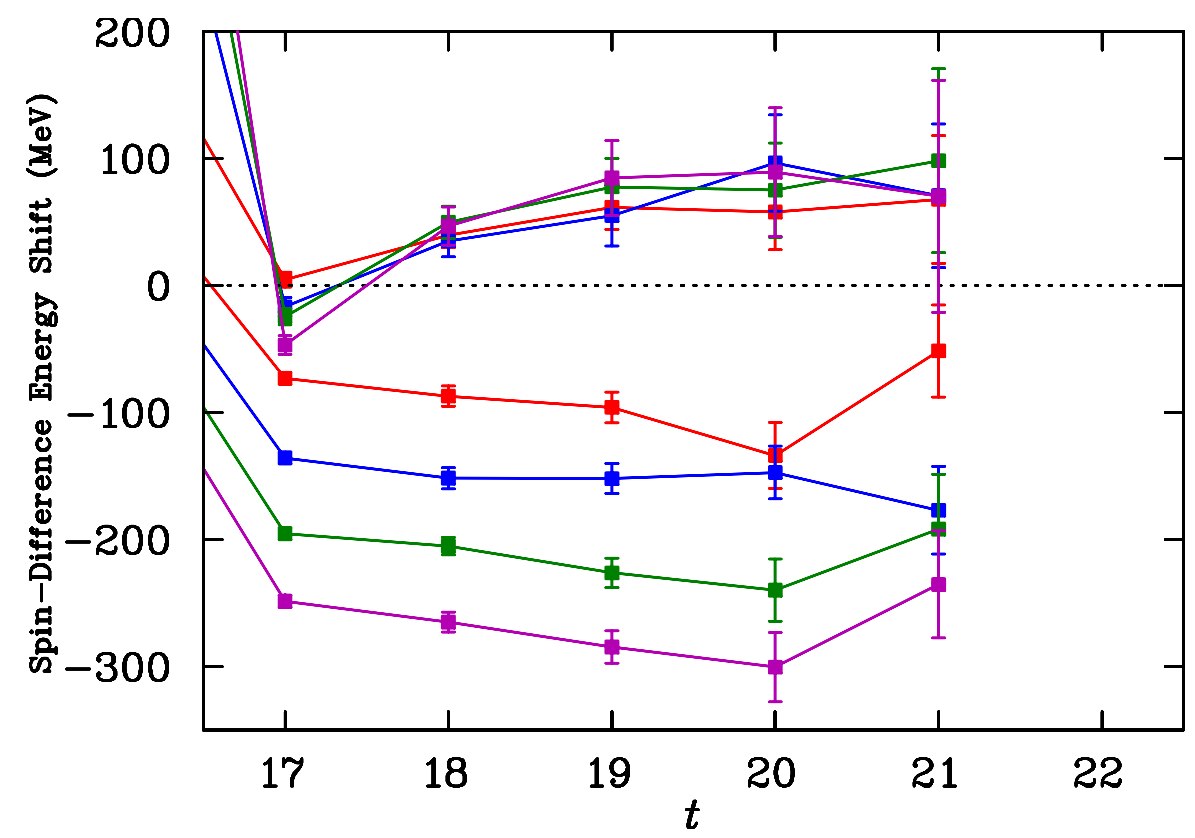

Figure 8.17: Spin-difference energy shift plot for odd-parity proton states from the variational analysis at $\kappa=0.13770$. Two states are illustrated for four different field strengths. State 1 is the negative energy shifts and state 2 is the positive shifts. The curves are ordered with the magnitude of the shifts increasing with the strength of the magnetic field for state 1. For state 2 the points are almost all degenerate. 


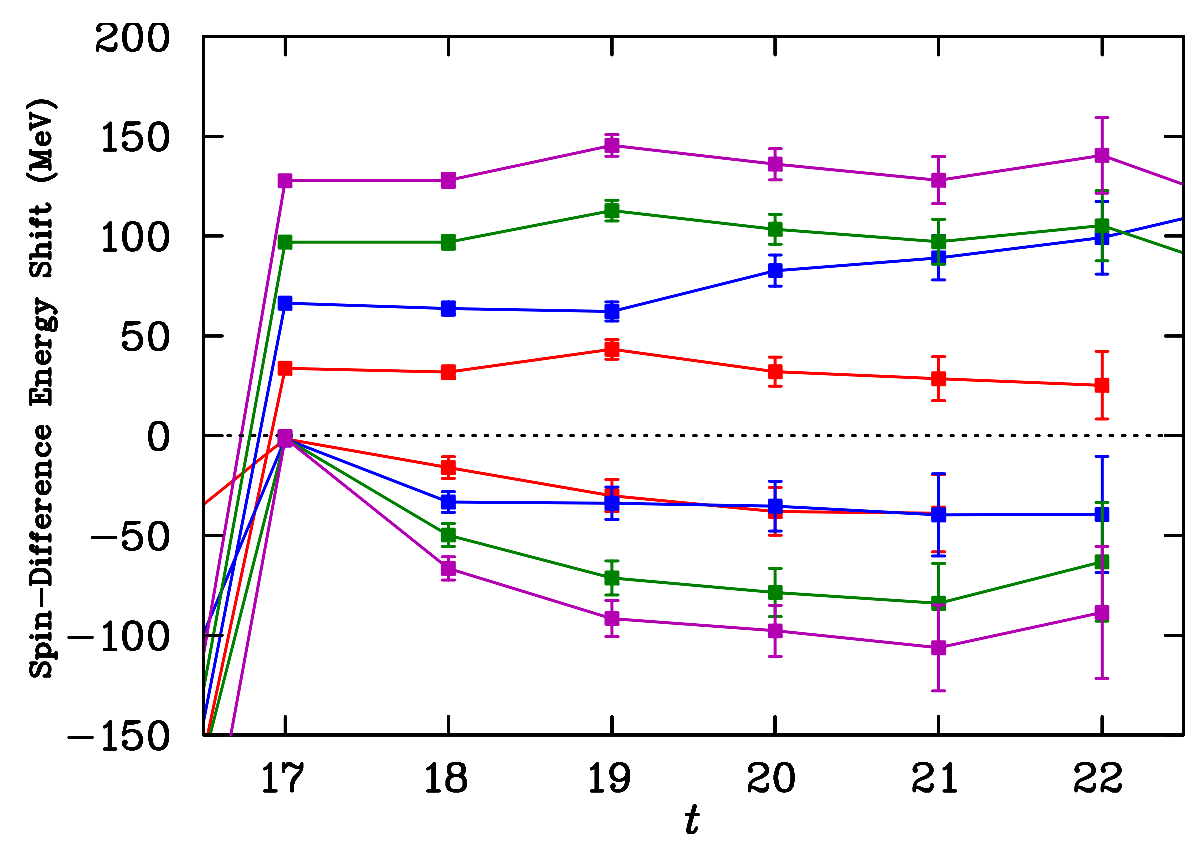

Figure 8.18: Spin-difference energy shift plot for odd-parity neutron states from the variational analysis at $\kappa=0.13700$. Two states are illustrated for four different field strengths. State 1 is the positive energy shifts and state 2 is the negative shifts. The curves are ordered with the magnitude of the shifts increasing with the strength of the magnetic field as of time slice 18 .

difficulties with the second field strength not following the trend we expect, especially at $\kappa=0.13754$ and $\kappa=0.13727$ where it has a smaller shift than the first field for state two.

\subsubsection{Fits to the field}

Figure 8.22 shows the fits of the spin-difference mass shifts to the magnetic field strength for the two lowest lying odd-parity proton states. As in the even-parity case we used two fits, a one parameter fit to the first two points and a two parameter fit to all the points. For the first state there is very good agreement between the two fits, with very little cubic contribution apparent at the heavier masses. For the second state there is still not that much difference, due to the fact that in cases where the second field strength point sits higher than expected, the first field strength sits lower than expected in 


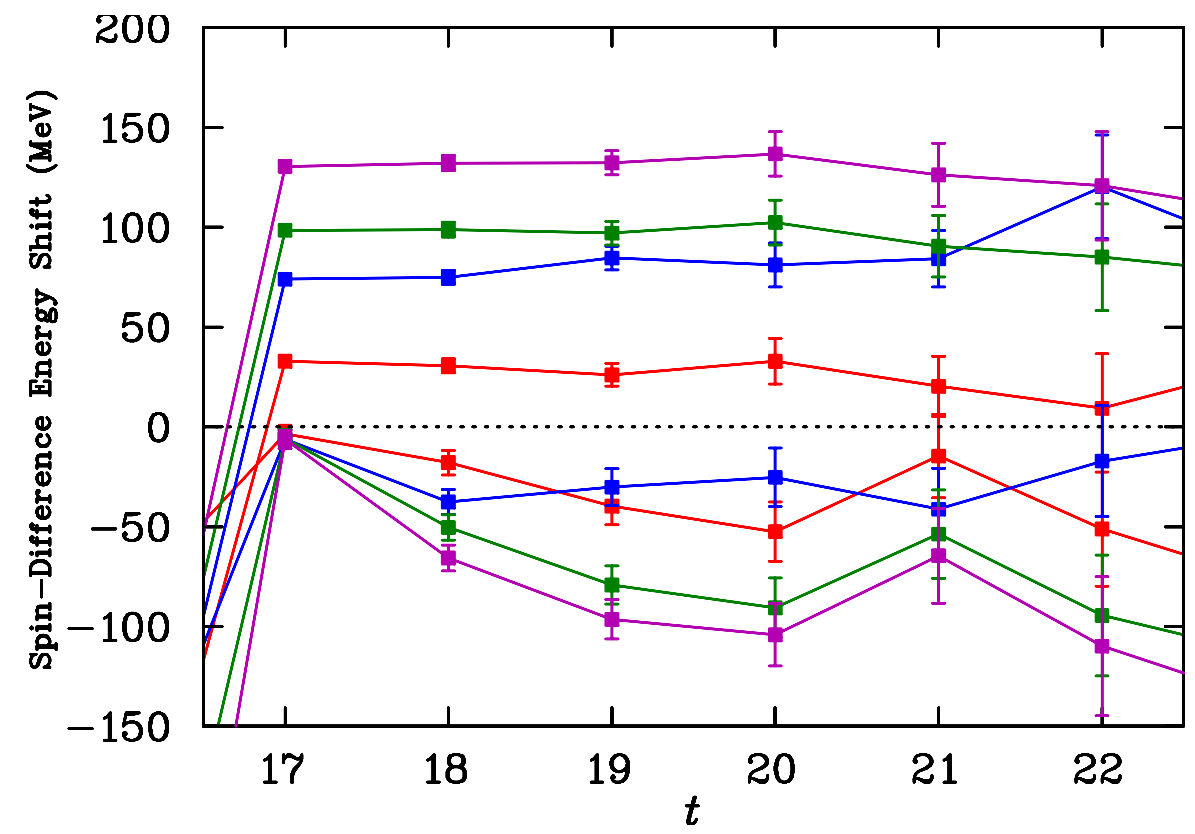

Figure 8.19: Spin-difference energy shift plot for odd-parity neutron states from the variational analysis at $\kappa=0.13727$. Two states are illustrated for four different field strengths. State 1 is the positive energy shifts and state 2 is the negative shifts. The curves are ordered with the magnitude of the shifts increasing with the strength of the magnetic field as of time slice 18 . 


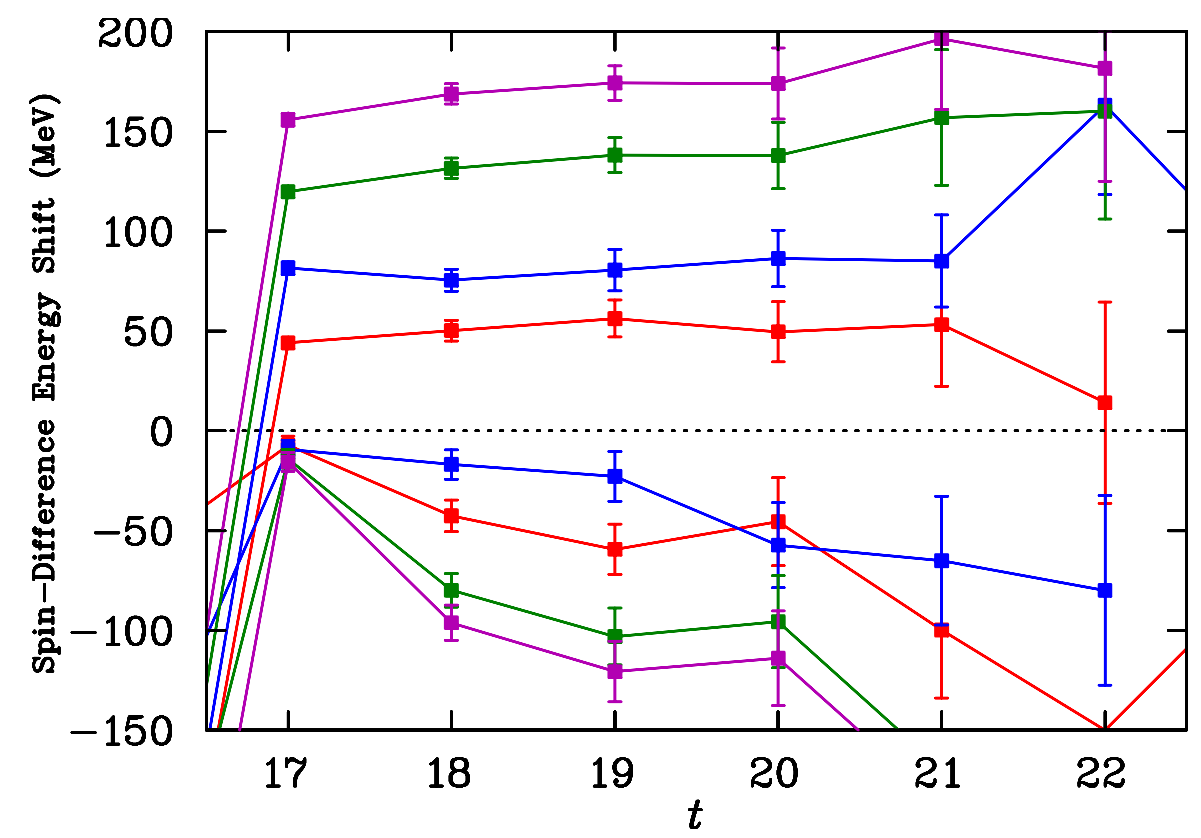

Figure 8.20: Spin-difference energy shift plot for odd-parity neutron states from the variational analysis at $\kappa=0.13754$. Two states are illustrated for four different field strengths. State 1 is the positive energy shifts and state 2 is the negative shifts. The curves are ordered with the magnitude of the shifts increasing with the strength of the magnetic field for state 1. For state 2 the first and second field strengths are switched in the region of 18-19. 


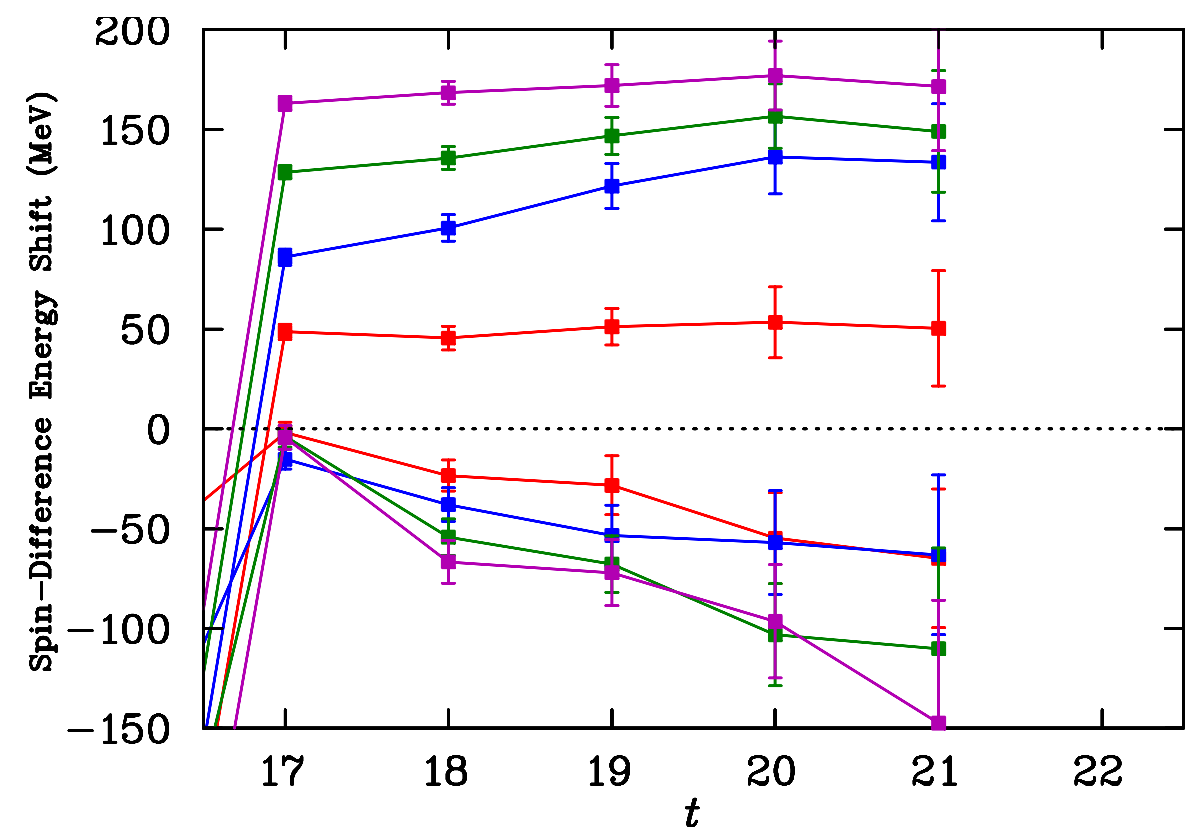

Figure 8.21: Spin-difference energy shift plot for odd-parity neutron states from the variational analysis at $\kappa=0.13770$. Two states are illustrated for four different field strengths. State 1 is the positive energy shifts and state 2 is the negative shifts. The curves are ordered with the magnitude of the shifts increasing with the strength of the magnetic field as of time slice 18 . 
a manner which mostly compensates.

Figure 8.23 shows the same fits for the odd-parity neutron states. Here the first state is again easy to fit, with almost zero cubic contribution visible except at the lightest quark mass. The second state has the same trend of the second field strength point being quite different from the predicted value, but with the first point varying in the opposite direction in a manner that gives fairly clear fits. The discrepancy in the values for the second state at the second field strength could be due to excited state contamination since we have only used a $2 \times 2$ correlation matrix here. Although the odd-parity spectrum shows a large gap between the two states we are examining and the next lightest state [42], a large magnetic moment could lead to an energy shift in the presence of a magnetic field which lowers the energy enough to interfere with the states we are considering. Future study using more sources may yield an improved result.

\subsubsection{Magnetic moment as a function of pion mass}

Figure 8.24 shows the odd-parity proton magnetic moment values as well as the even-parity values for comparison. The first odd-parity state, which we take to be the $S_{11}^{+}(1535)$ follows a similar trend to the even-parity ground state, but at a slightly smaller magnitude. The values for the second state are less clear, with no obvious trend and large uncertainties. Due to the relative difficulty in fitting the energy shifts and in particular the second field strength these results can only be considered exploratory. Despite this we can make some strong statements about the magnetic moment of this state, which we take to be the $S_{11}^{+}(1650)$. It clearly has a negative sign, opposite to that of the lowest lying even and odd-parity states. The magnitude of the moment is less than that of the first state but quite distinct from zero.

Figure 8.25 displays the moment values for the neutron states. The situation is very similar to that of the proton states. The first state $\left(S_{11}^{0}(1535)\right)$ has a gentle downward trend and a magnitude slightly smaller than that of the even-parity ground state. Here we used a simple linear extrapolation to get an idea of the trend to the physical pion mass. The second odd-parity nucleon state $\left(S_{11}^{0}(1650)\right)$ appears to have a mostly flat trend, although due to the large errors the trend is not at all definitive. The sign is clearly positive, opposite that of the other neutron states, and the magnitude is less than that found for the lowest lying odd-parity state. 

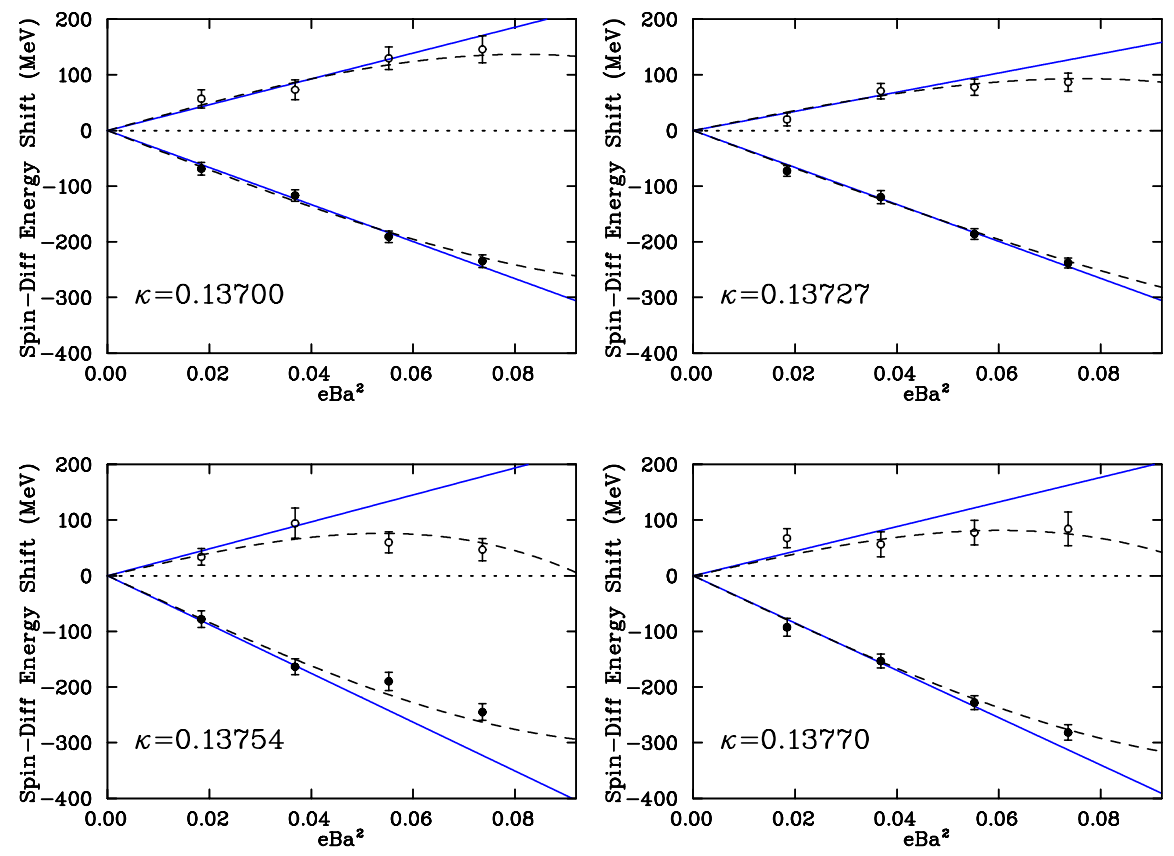

Figure 8.22: Fits of the spin-difference mass shift to the magnetic field strength for the two lowest lying odd-parity proton states. The solid line is a linear fit to the first two points and the dashed line is a linear plus cubic fit to all four points. State 1 is illustrated by the negative values while state 2 is the positive values. 

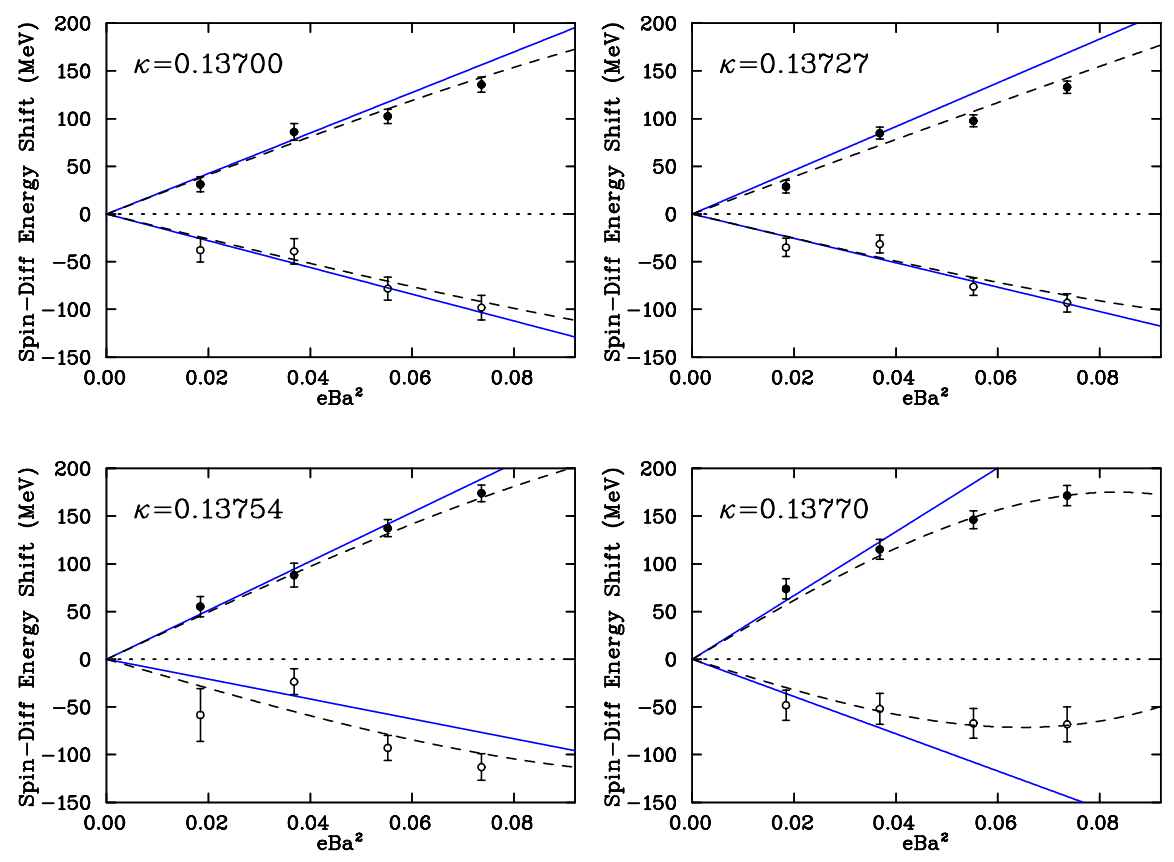

Figure 8.23: Fits of the spin-difference mass shift to the magnetic field strength for the two lowest lying odd-parity neutron states. The solid line is a linear fit to the first two points and the dashed line is a linear plus cubic fit to all four points. State 1 is illustrated by the positive values while state 2 is the negative values. 


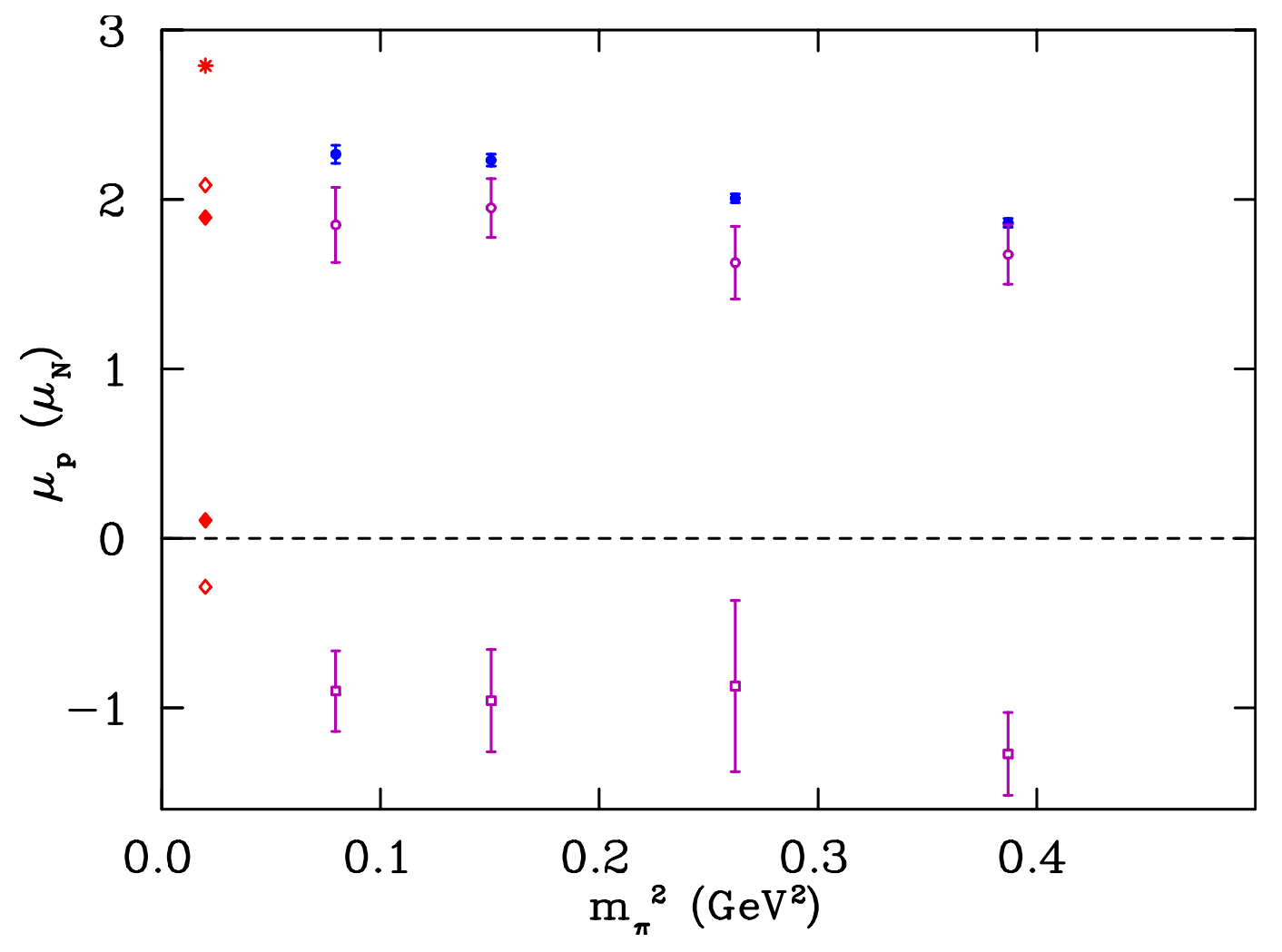

Figure 8.24: Magnetic moments of the proton and its odd-parity excitations as a function of pion mass. The solid circles are the even-parity results presented previously, the hollow circles are the first odd-parity state and the hollow squares are the second odd-parity state. At the far left the asterisk indicates the experimental value for the ground state [27] and the diamonds indicate the quark model predictions as described in Table 8.4. 


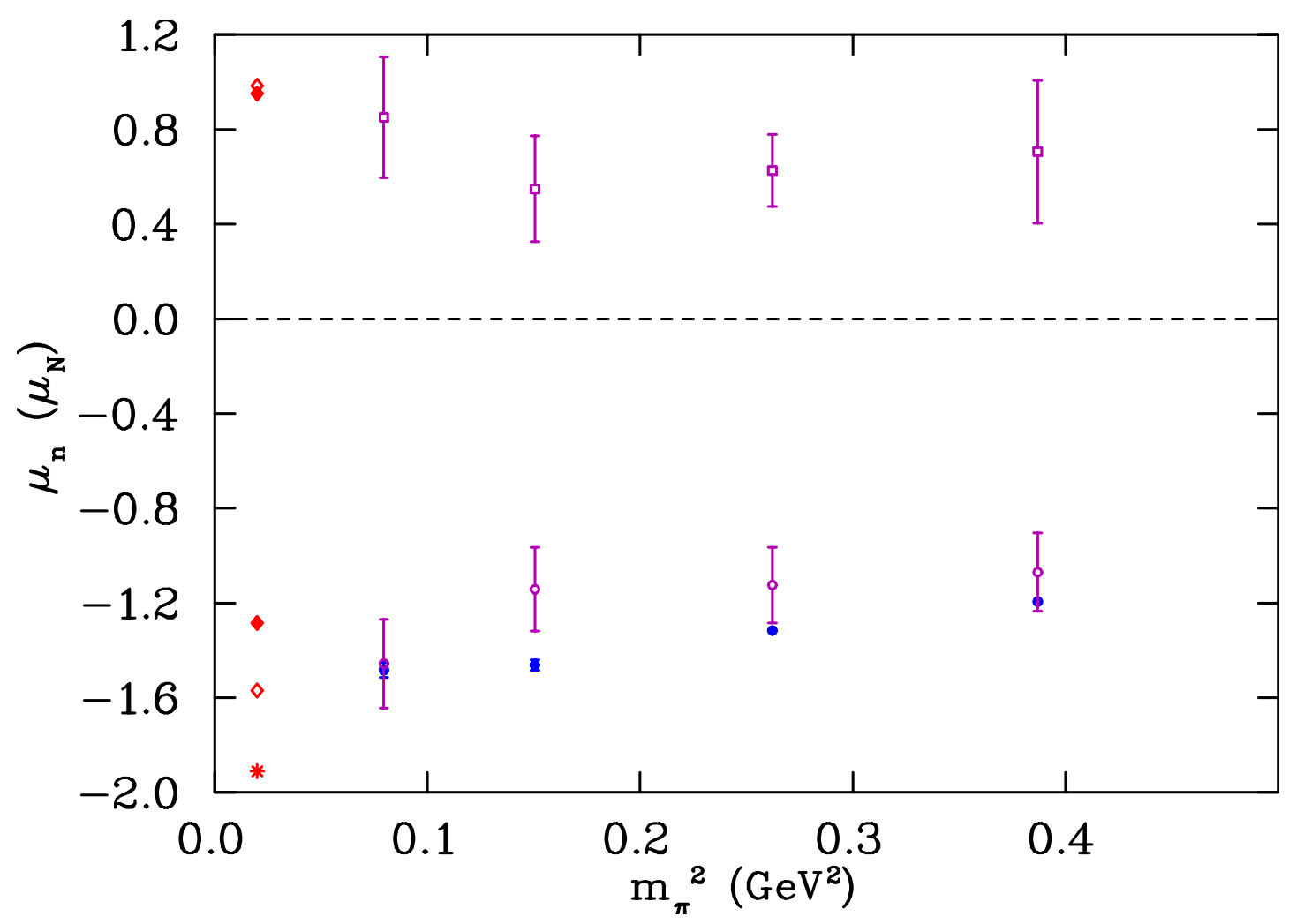

Figure 8.25: Neutron magnetic moments as a function of pion mass. The solid circles are the even-parity results presented previously, the hollow circles are the first odd-parity state and the hollow squares are the second odd-parity state. At the far left the asterisk indicates the experimental value for the ground state [27] and the diamonds indicate the quark model predictions as described in Table 8.4. 


\subsubsection{Quark model prediction}

As in the even-parity case it is possible to calculate the magnetic moment of the odd-parity nucleon using a constituent quark model. This value is much more valuable in this case because there do not exist any experimental results for the magnetic moment of the odd-parity proton or neutron states at present. Therefore the quark model prediction is useful for giving us a framework to compare our results within.

In the $S U(6)$ constituent quark model the lowest lying odd-parity states of the nucleon are $\left|N^{2} P_{1 / 2}\right\rangle$ and $\left|N^{4} P_{1 / 2}\right\rangle$, where the notations ${ }^{2} P_{1 / 2}$ and ${ }^{4} P_{1 / 2}$ indicate total quark spin $S=1 / 2,3 / 2(2 S+1=2,4)$, orbital angular momentum $L=1$, and total angular momentum $J=1 / 2$. The wave-functions of these states can be written explicitly,

$$
\begin{aligned}
\left|N^{2} P_{1 / 2}\right\rangle= & \frac{1}{\sqrt{2}} \sum_{m_{l} m_{s}}\left\langle 1 \frac{1}{2} m_{l} m_{s} \mid \frac{1}{2} \frac{1}{2}\right\rangle \\
& \times\left\{\psi_{1 m_{l}}^{\rho}\left[\frac{1}{\sqrt{2}}\left(\chi_{m_{s}}^{\lambda} \phi^{\rho}+\chi_{m_{s}}^{\rho} \phi^{\lambda}\right)\right]+\psi_{1 m_{l}}^{\lambda}\left[\frac{1}{\sqrt{2}}\left(\chi^{\rho} m_{s} \phi^{\rho}+\chi_{m_{s}}^{\lambda} \phi^{\lambda}\right)\right]\right\}, \\
\left|N^{4} P_{1 / 2}\right\rangle= & \frac{1}{\sqrt{2}} \sum_{m_{l} m_{s}}\left\langle 1 \frac{3}{2} m_{l} m_{s} \mid \frac{1}{2} \frac{1}{2}\right\rangle\left[\psi_{1 m_{l}}^{\rho} \chi_{m_{s}}^{s} \phi^{\rho}+\psi_{1 m_{l}}^{\lambda} \chi_{m_{s}}^{s} \phi^{\lambda}\right],
\end{aligned}
$$

where $\psi, \chi$, and $\phi$ denote the spatial, spin, and flavour wave-functions. The superscripts $s$ or $\rho(\lambda)$ indicate that they are totally symmetric among the three quarks, or mixed-symmetric, odd (even) under the exchange of the first two quarks.

The observed physical odd-parity nucleon resonances are mixtures of these $S U(6)$ spin-flavour states,

$$
\begin{aligned}
& \left|S_{11}(1535)\right\rangle=\left|N^{2} P_{1 / 2}\right\rangle \cos \theta-\left|N^{4} P_{1 / 2}\right\rangle \sin \theta \\
& \left|S_{11}(1650)\right\rangle=\left|N^{2} P_{1 / 2}\right\rangle \sin \theta+\left|N^{4} P_{1 / 2}\right\rangle \cos \theta
\end{aligned}
$$

The magnetic moment of these states is then calculated using the magnetic moment operator,

$$
\boldsymbol{\mu}=\boldsymbol{\mu}^{S}+\boldsymbol{\mu}^{L}
$$

where $\boldsymbol{\mu}^{S}$ indicates the magnetic moment due to the intrinsic spin of the quark and $\boldsymbol{\mu}^{L}$ is the part due to the orbital angular momentum of the quark. The magnetic moment for the $S_{11}(1535)$ and $S_{11}(1650)$ therefore has terms from the spin angular momentum and orbital angular momentum of the 
$S U(6)$ states, plus a term from the spin angular momentum of the mixing of the two $S U(6)$ spin-flavour states (the orbital angular momentum of the states is orthogonal and so has no mixing term). This method was used to calculate the magnetic moment in Ref. [44].

The quark model was expanded upon by another group in Ref. [45]. They added contributions from the spin and orbital angular momentum of the sea quarks and introduced a Goldstone boson which is emitted in a P-wave state $\left(\left\langle l_{z}\right\rangle=1\right)$. The values predicted by each model for the magnetic moment are given in Table 8.4. For three of the four states the additions in Ref. [45] serve to increase the magnitude of the moment by a small amount. For the $S_{11}^{+}(1650)$ however, the additional terms led to a moment with the opposite sign to that of the first prediction.

It is interesting to compare these results with our lattice results to evaluate the merit of the expanded model. The model predictions can be seen on Figures 8.24 and 8.25, with the solid diamonds representing Ref. [44] and the hollow diamonds representing Ref. [45]. For the lowest lying odd-parity proton and neutron states we had extrapolated values of $2.0(3) \mu_{N}$ and $-1.4(2)$ $\mu_{N}$ respectively from a simple linear extrapolation. These values are both in agreement with the predictions of both models, with the central values falling between them.

Our world's first calculation of the second lowest-lying odd parity nucleon states allows us to bring some insight to the situation. Of particular interest is the $S_{11}^{+}(1650)$ value, where the signs of the model predictions differ. Our lattice results for the $S_{11}^{+}(1650)$ are negative, with the central value significantly larger in magnitude than in Ref. [45]. Our $S_{11}^{0}(1650)$ result has a slightly smaller central value than the two model values but agrees with them both well within errors. 
Table 8.4: Predictions for the magnetic moment of the two lowest lying oddparity nucleon states from the quark model of Ref. [44] and an expanded model of Ref. [45]. Values are in units of nuclear magnetons. The predictions are compared to our lattice results in Figures 8.24 and 8.25, with the solid diamonds representing Ref. [44] and the hollow diamonds representing Ref. [45]. Our lattice results are from a simple linear extrapolation for each of the four states.

\begin{tabular}{|c|c|c|c|}
\hline $\begin{array}{c}\text { Baryons Mass } \\
(\mathrm{MeV})\end{array}$ & $\begin{array}{r}\operatorname{NCQM~[44]} \\
\left(\mu_{N}\right)\end{array}$ & $\begin{array}{r}\mathrm{CQM}[45] \\
\left(\mu_{N}\right)\end{array}$ & $\begin{array}{r}\text { Lattice (this work) } \\
\left(\mu_{N}\right)\end{array}$ \\
\hline 1535 & 1.89 & 2.09 & $2.0(3)$ \\
\hline 1535 & -1.28 & -1.57 & $-1.4(2)$ \\
\hline 1650 & 0.11 & -0.29 & $-0.8(3)$ \\
\hline 1650 & 0.95 & 0.98 & $0.7(3)$ \\
\hline
\end{tabular}




\section{Chapter 9}

\section{Magnetic Polarisability}

\subsection{Introduction}

The magnetic polarisability is a property of a system of charged particles which measures the degree to which it deforms in the presence of a magnetic field. On the lattice we see this as a shift in the effective mass of a hadron proportional to $B^{2}$. Proton polarisabilities can be measured experimentally via Compton scattering [46-48]. The neutron polarisability is somewhat more difficult due to the absence of a neutron target and having no overall charge, but can be done indirectly by scattering low energy neutrons off a heavy nucleus or from deuteron cross sections $[49,50]$. These calculations rely on the the sum rule,

$$
\alpha+\beta=\frac{1}{2 \pi^{2}} \int_{0}^{\infty} d \omega \frac{\sigma_{\mathrm{tot}}(\omega)}{\omega^{2}},
$$

where $\alpha$ is the electric polarisability, $\beta$ is the magnetic polarisability, $\omega$ is the photon energy and $\sigma$ is the scattering cross-section. This results in anticorrelated errors for $\alpha$ and $\beta$. The error on the experimental value of magnetic polarisability is large for both the proton and neutron. We therefore have the opportunity to make a prediction for the physical values through lattice QCD. There is currently no other method for calculating polarisabilities on the lattice than the background field method.

However calculating the polarisabilities of a charged particle using the background field method is problematic. For the electric polarisability the presence of the field causes a force on the particle which complicates the static energy solution $[17,18]$. For magnetic polarisabilities we have the issue of the 
Landau levels, as described in Chapter 6. Landau levels make it impossible to extract a clean ground state energy using the typical zero momentum wave function projection. For this reason we do not present proton polarisability results in this work. In the future we hope to develop finite-volume based techniques which will allow us to deal with the Landau levels accurately.

Even for a neutral particle like the neutron the polarisability is more difficult to calculate than the magnetic moment. First of all this is because the polarisability is a $B^{2}$ effect, making it much smaller than the order $B$ moment effect at the small field strengths we are using. We also find that the spin-averaged energy shift used in calculating the polarisability takes a long time to plateau, making it difficult to fit. In the following we investigate the use of different sources and sinks in order to try and improve the plateau behaviour of the energy shifts.

\subsection{Method}

Recall again the energy-field relationship for a hadron in a uniform magnetic field $[9,11]$,

$$
E(B)=M-\boldsymbol{\mu} \cdot \mathbf{B}+\frac{e|B|}{2 M}-\frac{4 \pi}{2} \beta B^{2}+\mathcal{O}\left(B^{3}\right) .
$$

As discussed in Chapter 8, the sign of the magnetic moment term in this equation is opposite for spin-up and spin-down. We can take advantage of this to remove that term from the energy by taking the average of spinup and spin-down. Since we are now looking only at the neutron magnetic polarisability the Landau energy term $e|B| / 2 M$ is zero and can be ignored. This leaves us with just the bare, zero-field mass and the polarisability term in addition to higher order terms. As with the spin-difference used in the moment calculations there are multiple ways of constructing our spin-average from correlators, such as,

$$
\Delta E(B)=\left(G_{\uparrow}(B, t)_{f i t}-G_{\uparrow}(0, t)_{f i t}+G_{\downarrow}(B, t)_{f i t}-G_{\downarrow}(0, t)_{f i t}\right) / 2,
$$

or,

$$
\Delta E(B)=\frac{1}{2}\left(\left(\frac{G_{\uparrow}(B, t)}{G_{\uparrow}(0, t)}\right)_{f i t}+\left(\frac{G_{\downarrow}(B, t)}{G_{\downarrow}(0, t)}\right)_{f i t}\right) .
$$




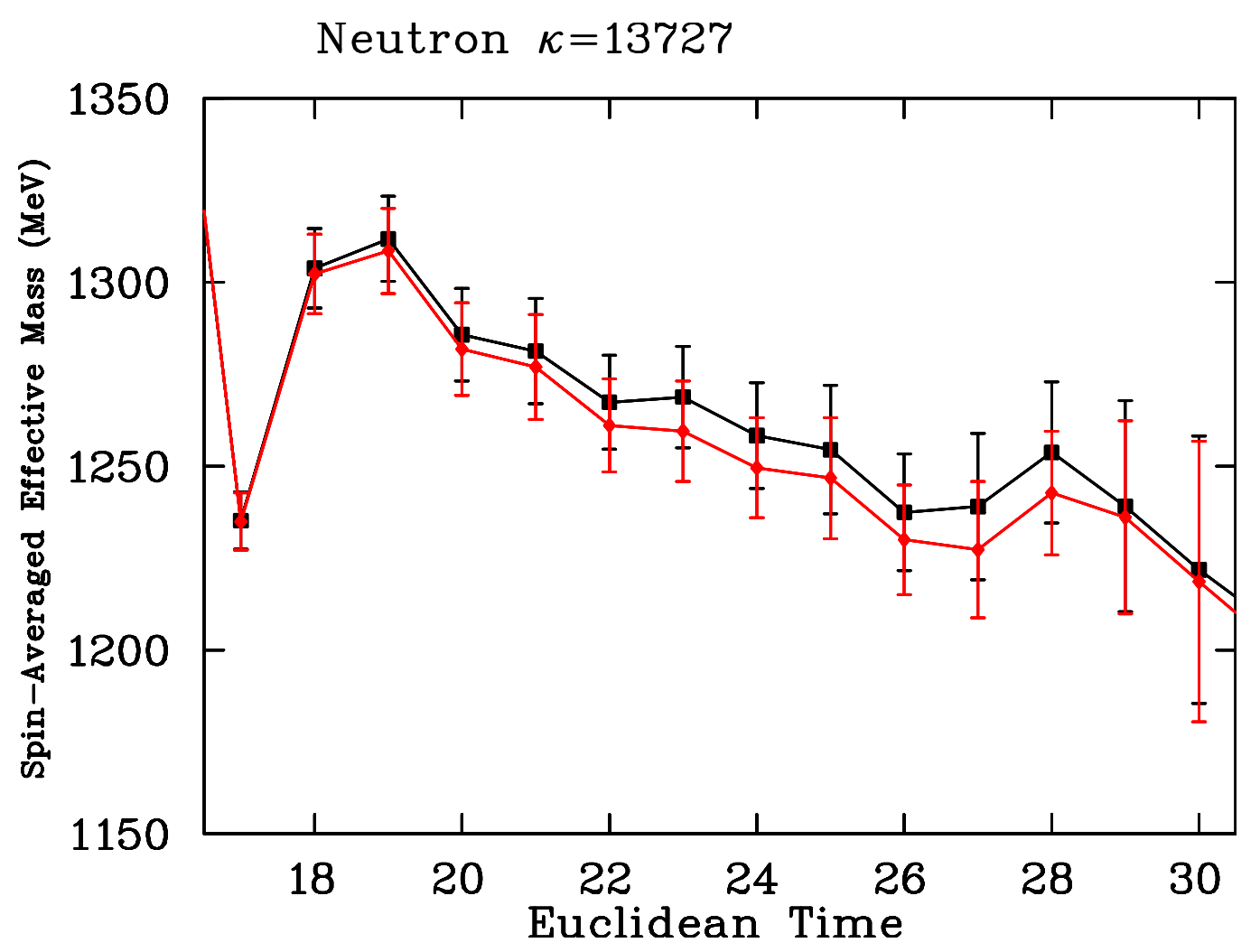

Figure 9.1: Spin-averaged effective mass for the neutron with zero field mass still included. The top line (squares) is for zero field and the bottom line (circles) is for the smallest non-zero field strength.

Here the subscript ${ }_{f i t}$ again indicates a fit of the effective energy. Using what we learned from the magnetic moment calculations, the best results come from using a single fit,

$$
\Delta E(B)=\frac{1}{2}\left(\frac{G_{\uparrow}(B, t)}{G_{\uparrow}(0, t)} \frac{G_{\downarrow}(B, t)}{G_{\downarrow}(0, t)}\right)_{f i t} .
$$

Making sure to combine field on and zero field correlation functions before fitting is even more important here than in the spin-difference case. Figure 9.1 shows the spin-averaged effective mass with the field off and field on. At each point in time the values agree within error because the shift due to the polarisability is smaller than the error in the overall energy. However, the uncertainties are highly correlated and largely cancel in taking the ratio. 
This provides a correlator directly related to the physical quantity of interest. Moreover, the covariance matrix based $\chi_{\text {dof }}^{2}$ provides a direct measure of the quality of this signal. Although Figure 9.1 displays a slight downward trend this is due to the close scale used to show the small effect. A plateau can easily be fit from $t=22$ to $t=30$ with a good $\chi_{\text {dof }}^{2}$ of 0.56 .

\section{$9.3 \quad$ Results}

\subsubsection{Effective energies}

We take the average of spin-up and spin-down correlation functions and remove the zero field mass using the ratio defined in Eq. (9.5). The resultant effective energies are shown in Figures 9.2 to 9.5. We see immediately that the behaviour is very different from what we saw for the spin-differences in Figures 8.2 through 8.5. Instead of being very flat right from the source, these effective energies all start at approximately zero and then decrease monotonically. Only the smallest field strength shows anything like a good plateau, all of the other higher field strengths are still decreasing up until the time when they are overtaken by statistical noise.

We can compare these for the neutron to proton spin-averaged energy shifts, given by Figure 9.6. The experimental value for the proton magnetic polarisability is very similar to the neutron, so we would expect the plots to be similar in the absence of other effects. Instead we see clear influence from the Landau levels, leading to a positive energy shift and much larger errors.

As they are, the neutron effective energies are extremely difficult to fit. In typical mass spectroscopy a lack of plateau at early times is generally due to excited state overlap in the correlator. Our bare effective mass plots illustrated in Fig. 9.1 do reveal a systematic drift in the energy suggesting some improvement in the interpolating field may be possible. Our previous technique of fitting at the earliest possible time that gives an acceptable value

for the $\chi_{\text {dof }}^{2}$ is not effective, because the only windows that give decent $\chi^{2}$ values are those that are fitting into the giant errors at the onset of noise.

\subsubsection{Source smearing}

Since these effective energies cannot be accurately fit we had to look for ways to improve them. We tried to improve our plateau behaviour using different 


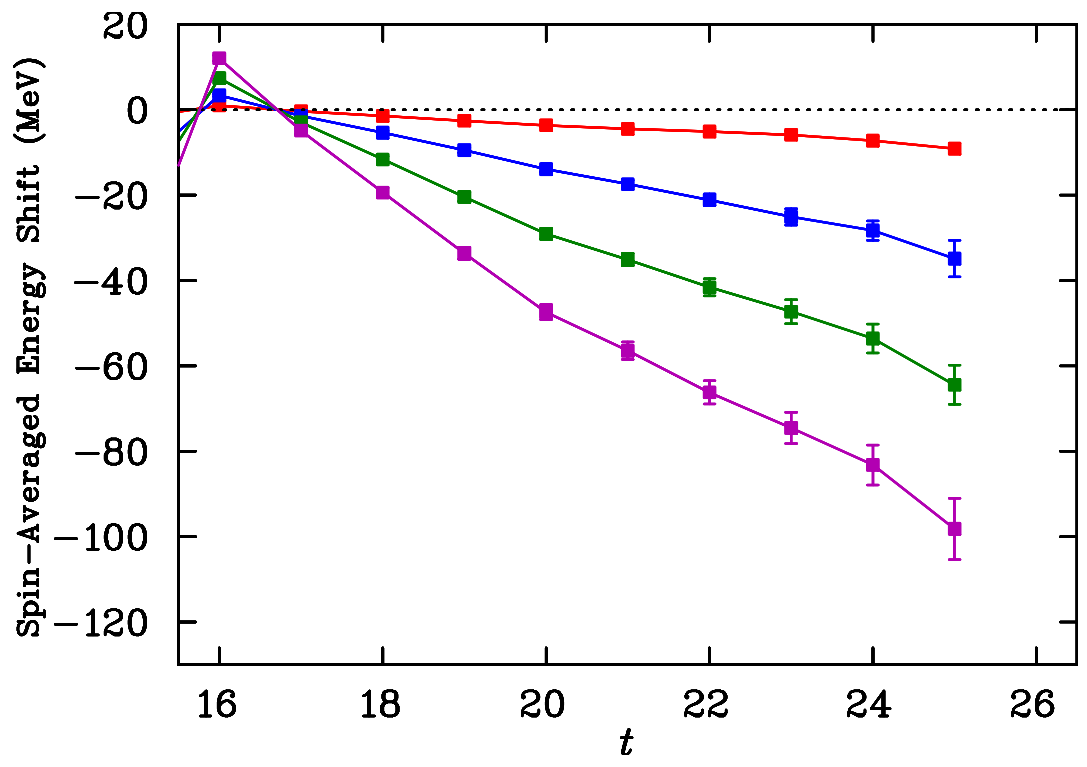

Figure 9.2: Spin-averaged energy shift proportional to the magnetic polarisability for the neutron at $\kappa=0.13700$. Field strengths go from top to bottom, weakest to strongest.

sources. We experimented with a number of different source smearings in addition to our usual 100 sweeps of smearing, by varying the number of sweeps while keeping the smearing fraction the same. We tried 16 and 35 sweeps of smearing as well as trying a point source. The results of the different smearings for spin-up and spin-down are shown in Figure 9.7. For the spin-average it was found that each of the smeared sources gave approximately the same result. The plots of spin-up and spin-down show that the smeared sources all agree with each other by time slice 24 , indicating that by then the majority of the excited state contributions have been suppressed. The point source starts with huge excited state contaminations and is approaching agreement with the smeared sources as it is overcome with noise.

One thing we noticed in Figure 9.7 is that for spin-up the best plateau behaviour comes from the source with 16 sweeps of smearing and for spindown the best behaviour comes from 100 sweeps of smearing. We therefore decided to combine these two smearings when constructing our spin-average. The result of this combination is shown in Figure 9.8. As expected the combined smearing results in a much improved behaviour, with a nice early 


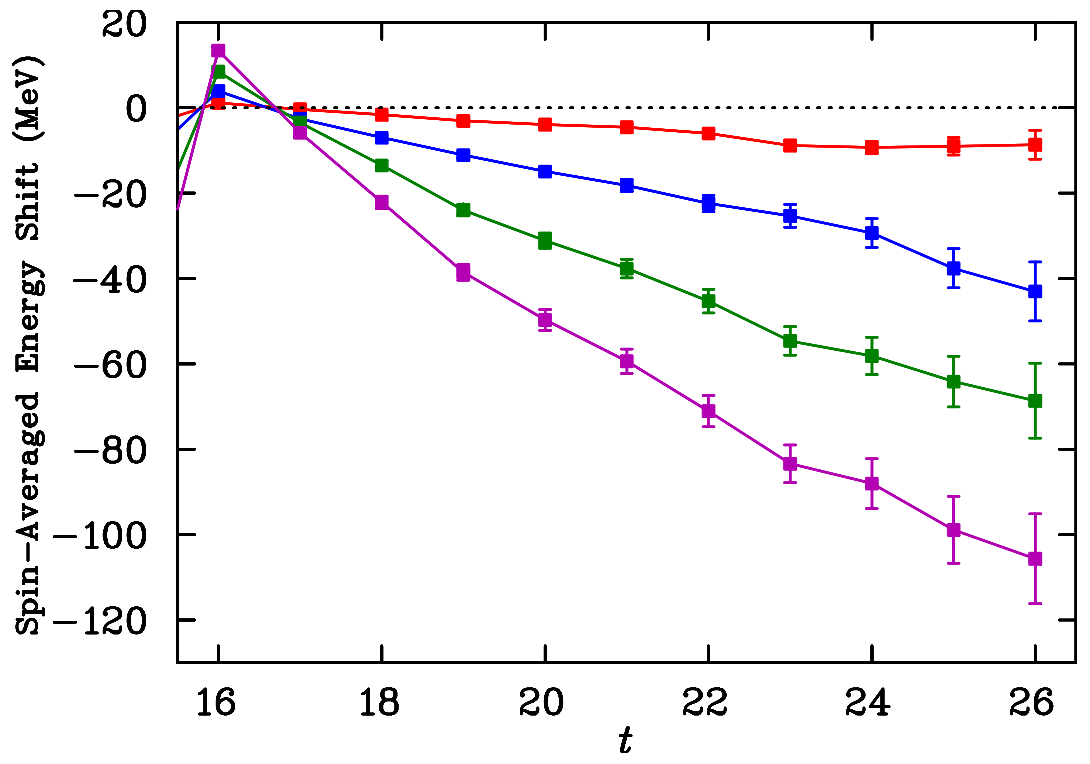

Figure 9.3: Spin-averaged energy shift proportional to the magnetic polarisability for the neutron at $\kappa=0.13727$. Field strengths go from top to bottom, weakest to strongest.

plateau up to time slice 24 where all the curves agree.

Taking the idea of combining smearings further, we can use the variational method to find the best possible combination for extracting the ground state. Details of the variational method can be found in Section 8.5.2, but the basic process is that we construct a correlation matrix from different sources and sinks,

$$
\begin{aligned}
G_{i j}(t) & =\sum_{\mathbf{x}} e^{i \mathbf{p} \cdot \mathbf{x}}\left\langle\Omega\left|\chi_{i} \bar{\chi}_{j}\right| \Omega\right\rangle \\
& =\sum_{\alpha} \lambda_{i}^{\alpha} \bar{\lambda}_{j}^{\alpha} e^{-E_{\alpha} t},
\end{aligned}
$$

then solve a pair of eigenvalue equations to get left and right eigenvectors,

$$
\begin{aligned}
{\left[\left(G\left(t_{0}\right)\right)^{-1} G\left(t_{0}+\Delta t\right)\right] u^{\alpha} } & =e^{-m_{\alpha} \Delta t} u^{\alpha} \\
v^{\alpha}\left[G\left(t_{0}+\Delta t\right)\left(G\left(t_{0}\right)\right)^{-1}\right] & =e^{-m_{\alpha} \Delta t} v^{\alpha}
\end{aligned}
$$

which project out individual eigenstates,

$$
v_{i}^{\alpha} G_{i j}(t) u_{j}^{\beta}=\delta^{\alpha \beta} \lambda^{\alpha} \bar{\lambda}^{\beta} e^{-m_{\alpha} t} .
$$




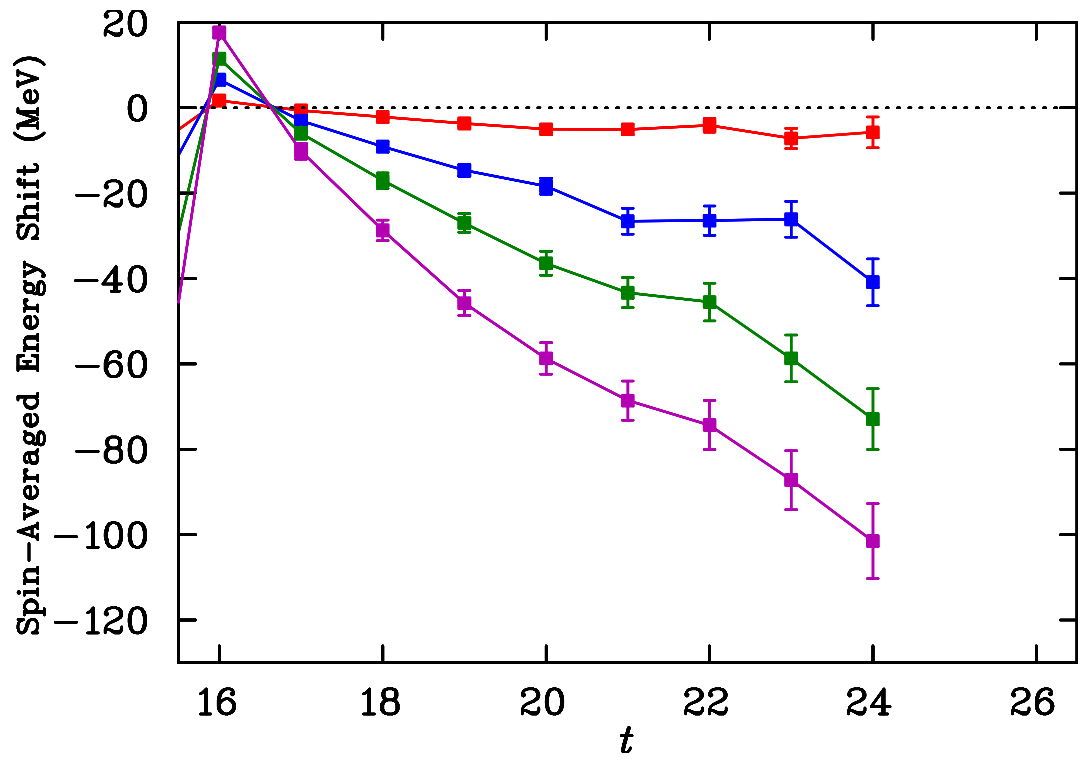

Figure 9.4: Spin-averaged energy shift proportional to the magnetic polarisability for the neutron at $\kappa=0.13754$. Field strengths go from top to bottom, weakest to strongest.

Using an $N \times N$ correlation matrix it is possible to isolate up to $N$ different states depending on how well your interpolator basis spans the state space. We could in theory apply background field method techniques to the excited states, although the signal tends to get weaker quickly as you go to higher excited states. For this purpose though we are only interested in the ground state.

We applied the variational analysis using a $2 \times 2$ correlation matrix of sources and sinks with 16 and 100 sweeps of smearing at the heaviest mass and smallest field strength. As discovered in Section 8.5 the eigensystem must be solved separately for spin-up field off, spin-down field off, spin-up field on, and spin-down field on. We construct our spin-average with bare mass subtracted from the projected correlation functions. The eigenvectors are approximately the same for each of the different spin-field combinations. Thanks to this the correlation between the errors is mostly maintained and the errors stay small.

Figure 9.9 shows a comparison between the plain 100 sweeps of smearing correlation functions and the projected ground state from the variational 


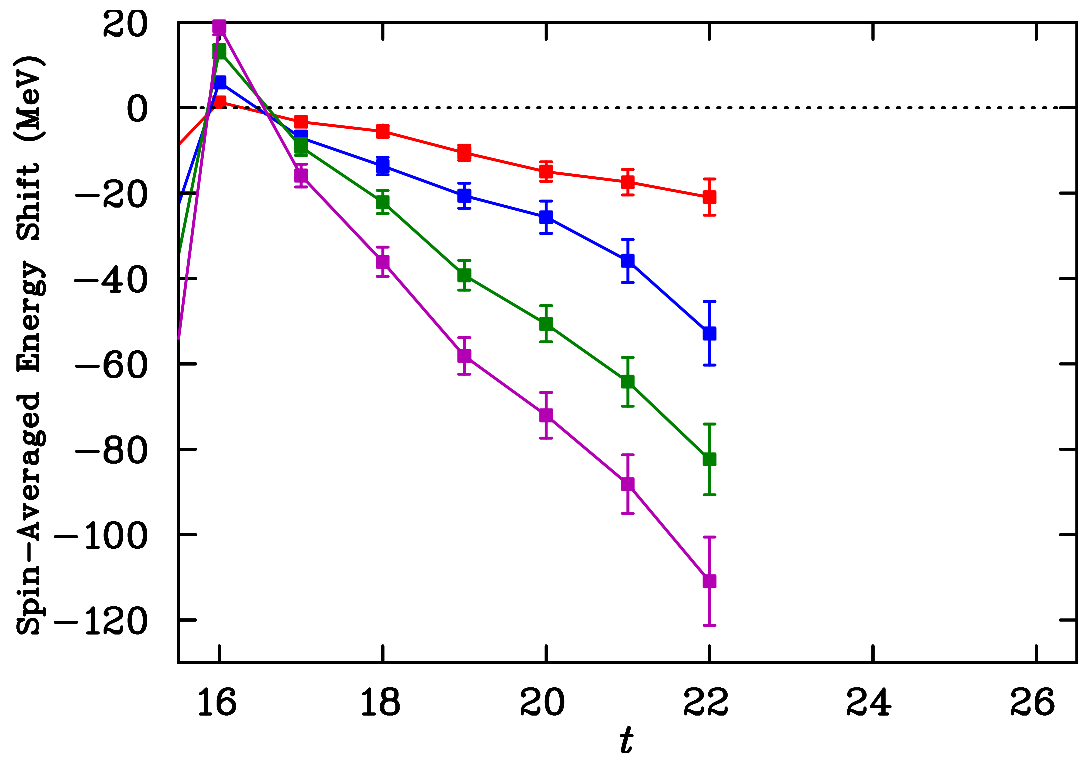

Figure 9.5: Spin-averaged energy shift proportional to the magnetic polarisability for the neutron at $\kappa=0.13770$. Field strengths go from top to bottom, weakest to strongest.

method using $t_{0}=18$ and $\Delta t=3$. The projected ground state follows a similar trajectory to the normal correlation function. We do not see the good asymptotic behaviour that was exhibited in Figure 9.8. This indicates there is more than a single excited state contamination and significantly more sources are required. The projected energy shift does show errors that grow slightly more slowly than those from the normal correlation function which could lead to an improved fit. While some improvement is observed in the $2 \times 2$ analysis, in that the projected correlator sits below the single 100 sweep correlator, we defer a more ambitious examination of correlation matrix techniques to the future.

In addition to the 2 by 2 correlation matrix using 16 and 100 sweeps of smearing we also tried some other operator bases. We tried different interpolator forms, with a $2 \times 2$ matrix of $\chi_{1}$ and $\chi_{2}$ at 100 sweeps of smearing. We tried a $3 \times 3$ matrix using the point source in addition to the 16 and 100 sweeps of smearing, and we tried a $4 \times 4$ matrix using 16 and 100 sweeps of smearing with both $\chi_{1}$ and $\chi_{2}$.

We tried a variety of values for the variational parameters $t_{0}$ and $\Delta t$. 


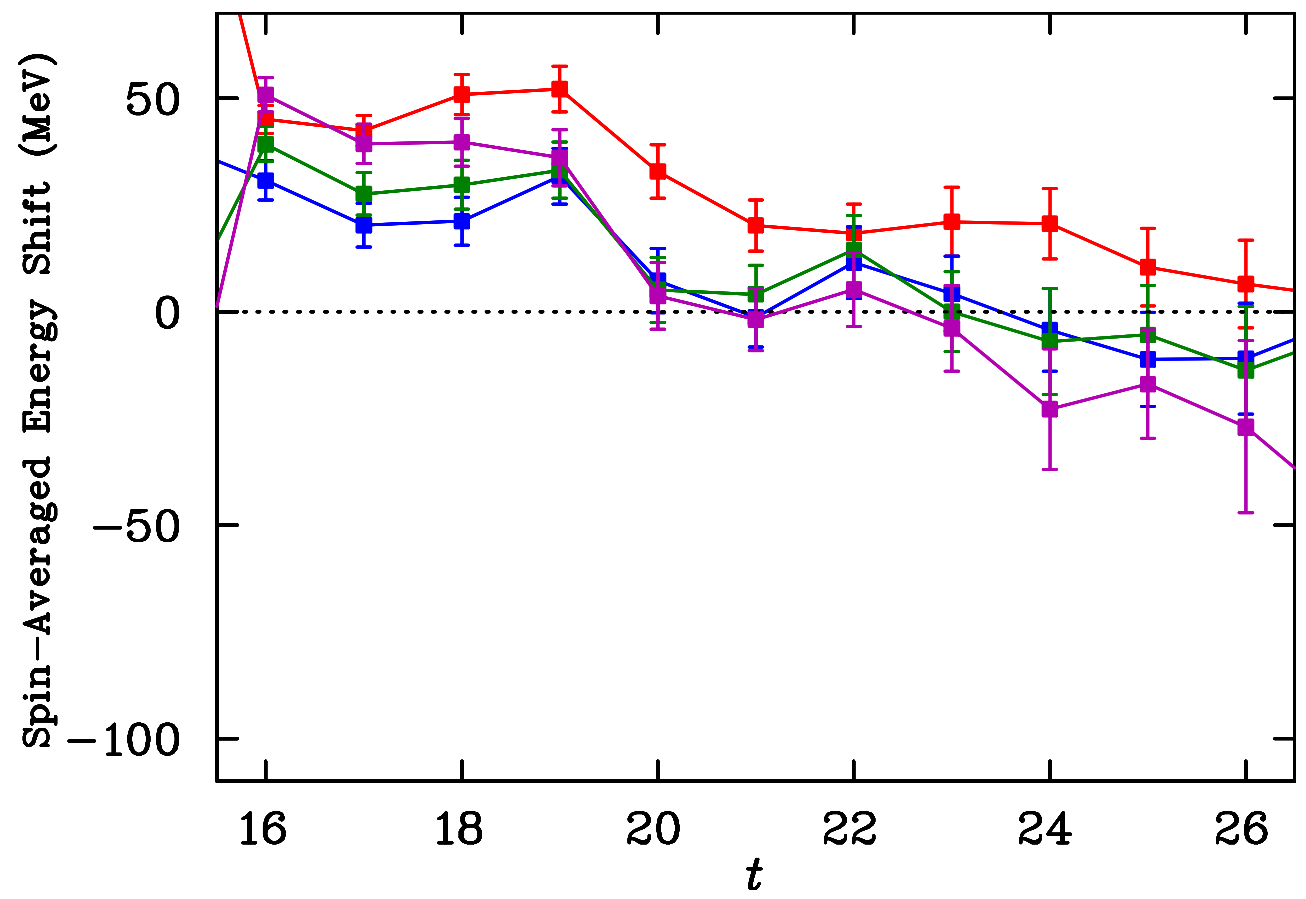

Figure 9.6: Proton spin-averaged energy shift for the heaviest quark mass at all field strengths. The top line is the smallest field strength, with the other three agreeing well within errors for most of the relevant time frame. 

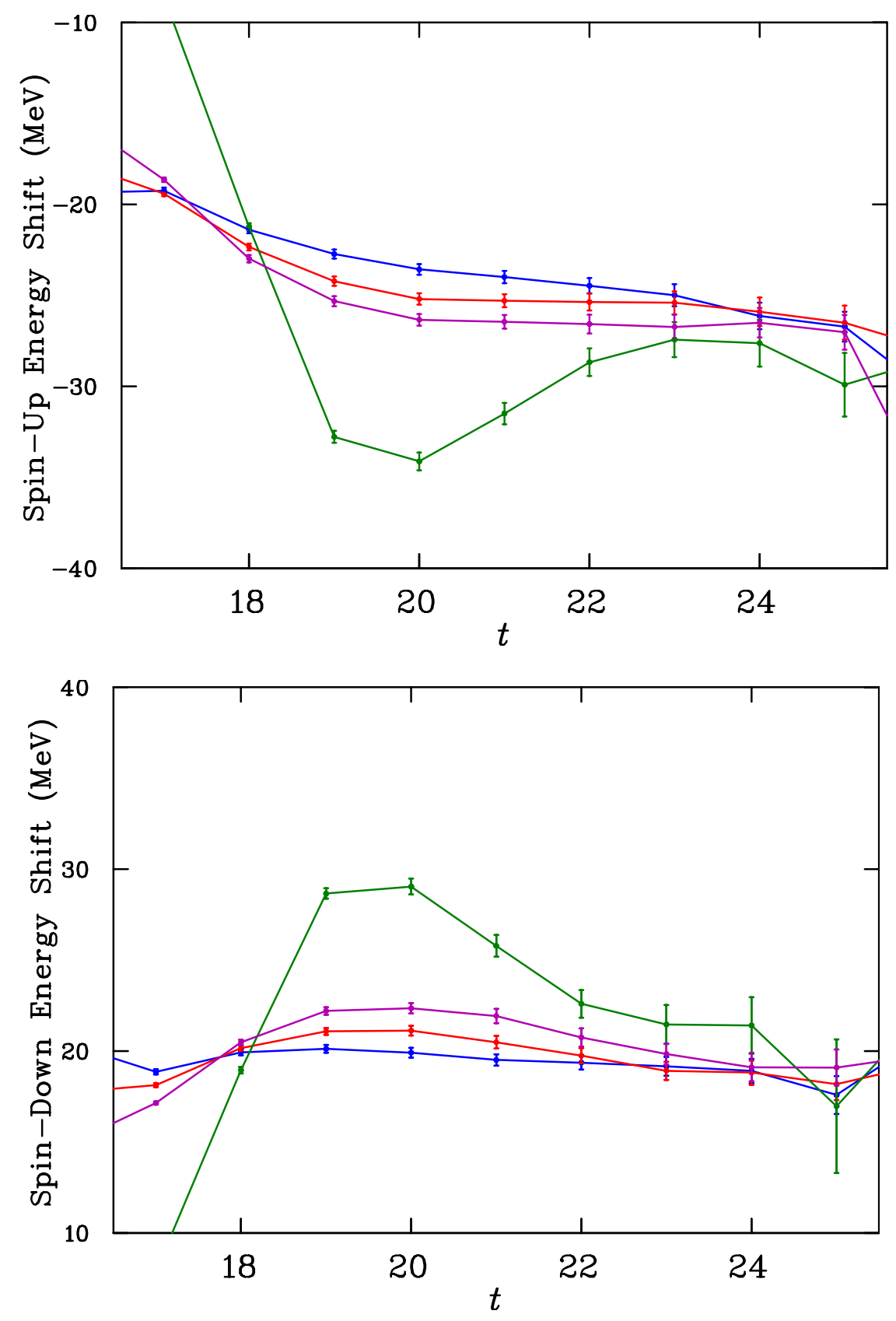

Figure 9.7: The shift in the energy due to the smallest magnetic field at the heaviest mass for spin-up (top) and spin-down (bottom). The curves represent different smeared fermion sources. For spin-up, from top to bottom at $t=20$ : 100 sweeps, 35 sweeps, 16 sweeps and point source. For spin-down the order is reversed. 


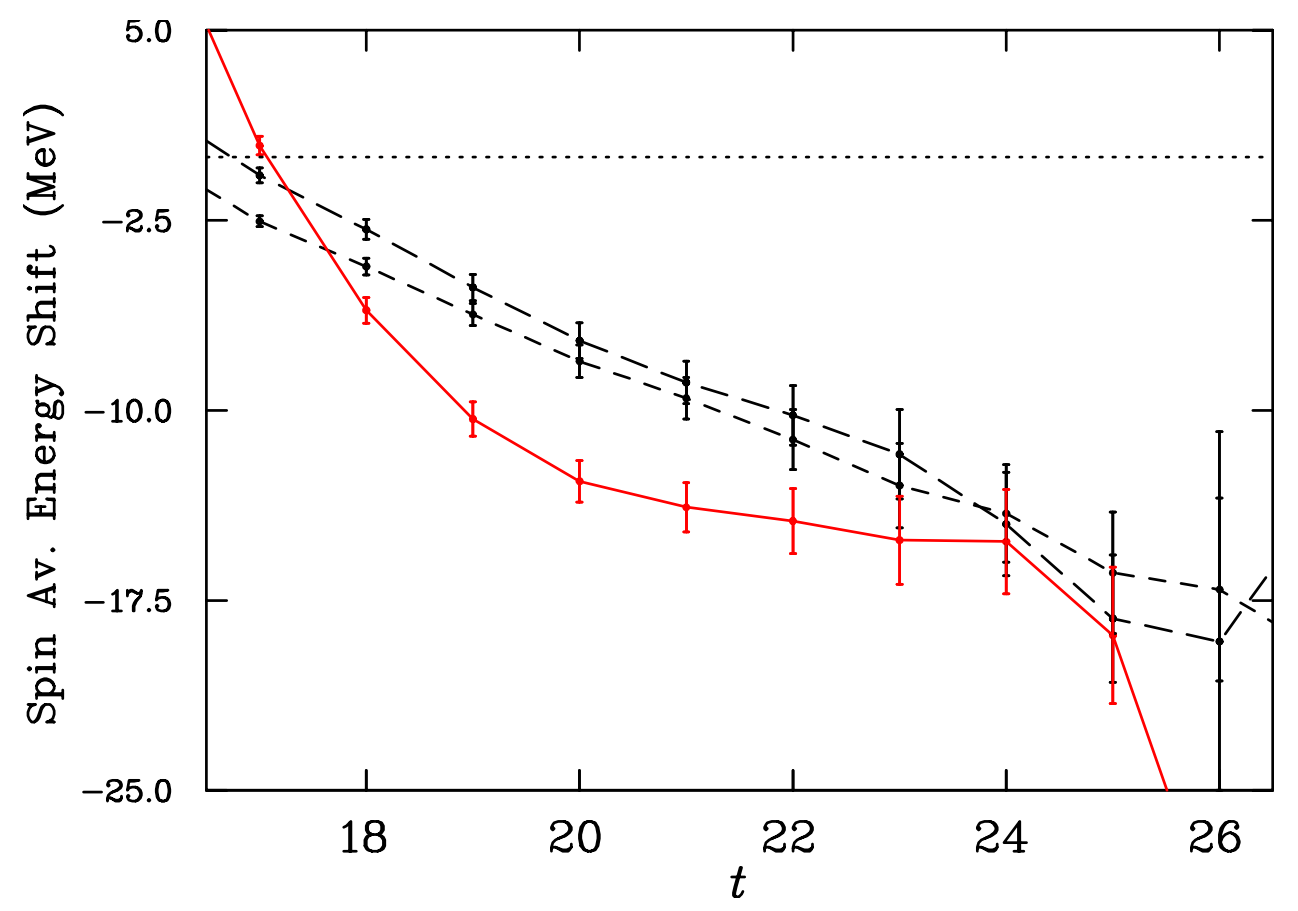

Figure 9.8: Spin-average energy shift for the neutron at the smallest field strength and heaviest mass. The dashed lines represent the 100 sweeps of smearing and 16 sweeps of smearing sources. The solid line is from combining spin-up with 16 sweeps and spin-down with 100 sweeps. 


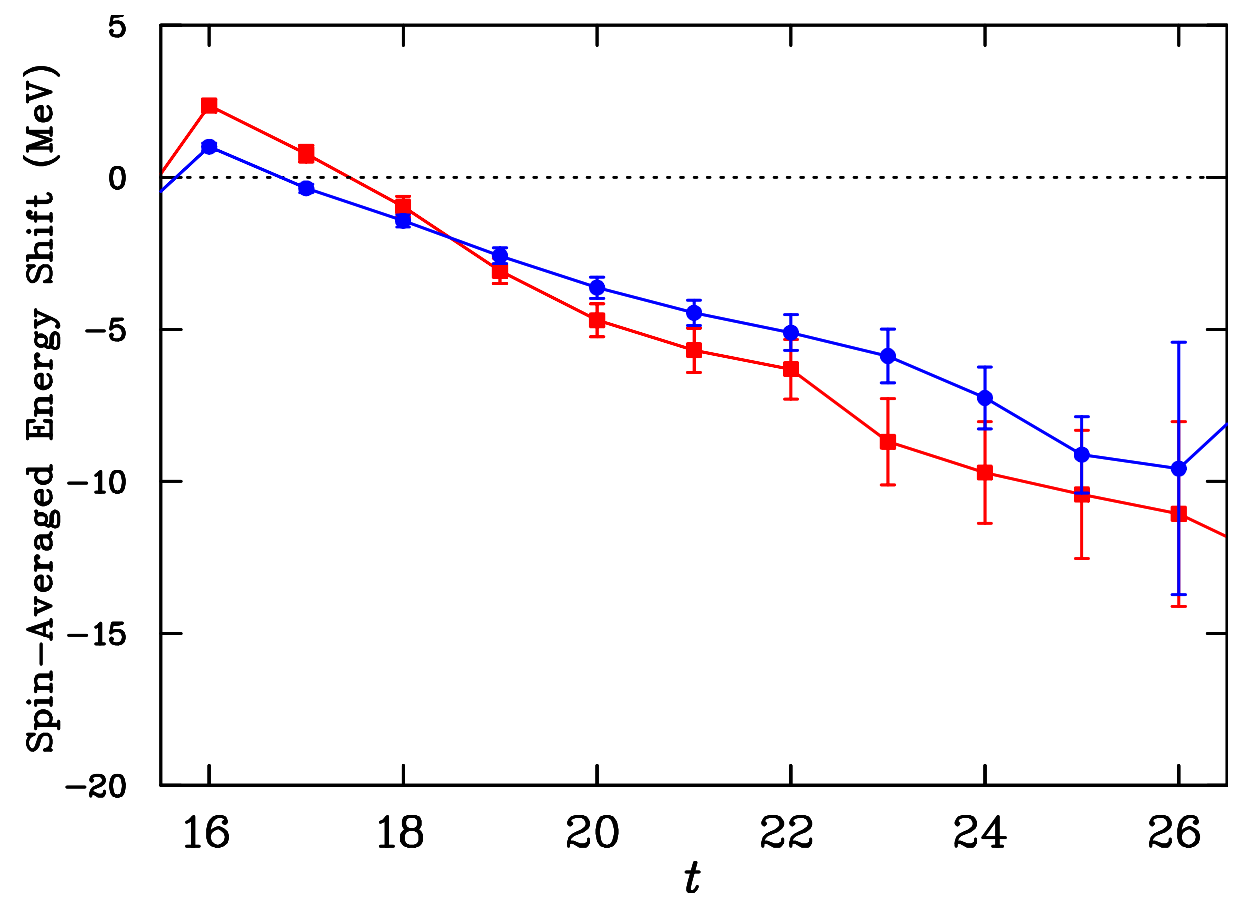

Figure 9.9: Spin-averaged energy shift for the neutron at the smallest field strength and heaviest mass. The squares are from a $2 \times 2$ correlation matrix with 16 and 100 sweeps of smearing at the source. The circles are with the usual 100 sweeps of source smearing. 
This included typical values of $t_{0}=17,18,19,20$ with $\Delta t=1,2,3,4$. We also tried using later times like $t_{0}=24,25$ in the area we might be hoping to fit. This did not significantly affect the projected energies at these times, but caused the time slices just after the source to have large uncertainties. None of these combinations led to any noticeable improvement in the plateau behaviour beyond the original $2 \times 2$ analysis.

Since we can not find any significant improvement in the energy shifts through the use of different smearings we need to choose a way of getting a best estimate for the energy shift from the effective energies we have. Looking

at the different smearings and combinations thereof we can see that all of the different effective energies agree well at time slice 24 for the heaviest mass. There are similar points at the other masses. The agreement makes it plausible that the ground state has been isolated and we use these points as our estimate for the effective energy shift associated with the polarisability and apply our fits to these values

The lack of early plateaus for the neutron despite the fact that there seems to be very little excited nucleon contributions is hard to explain. One hypothesis is that the effect is evidence that the neutron does actually experience Landau levels. Although the total charge of the hadron is zero, the charge radius of the particle is non-zero, due to the internal distribution of the quarks. It is therefore possible that the neutron does feel the magnetic field, but with a much smaller effective charge than the proton. This would lead to extremely closely spaced Landau levels which would decay smoothly in the effective mass. This seems to be consistent with what we see in our spin-averaged energy shift plots, but will require further study.

\subsubsection{Magnetic field dependence}

Figure 9.10 shows plots of the spin-averaged energy shift against the magnetic field strength. These points have significantly larger relative errors than the spin-difference points used in the magnetic moment calculations. The first field strength has significantly smaller errors than the others at all quark masses. This makes the first point the most important one in the fit. We fit the mass shift to a quadratic term which is proportional to the polarisability. As with the moment case the fit is constrained to go through zero. Again we see that there are clearly some higher order contributions which become large at the two largest field strengths. We therefore require an extra term in our polynomial to fit these points. In this case we add a quartic term since 


\begin{tabular}{c|cccc}
\hline \hline & \multicolumn{2}{|c}{2 point, 1 parameter } & \multicolumn{2}{c}{4 point, 2 parameter } \\
\hline$\kappa$ & $\beta_{n}\left(\mathrm{fm}^{3} \times 10^{-4}\right)$ & $\chi_{\text {dof }}^{2}$ & $\beta_{n}\left(\mathrm{fm}^{3} \times 10^{-4}\right)$ & $\chi_{\text {dof }}^{2}$ \\
\hline 0.13700 & $1.06(90)$ & 0.04 & $1.08(81)$ & 0.20 \\
0.13727 & $1.17(15)$ & 0.23 & $1.17(13)$ & 0.98 \\
0.13754 & $0.97(10)$ & 1.60 & $0.98(10)$ & 1.15 \\
0.13770 & $1.20(13)$ & 3.66 & $1.20(13)$ & 2.17 \\
\hline \hline
\end{tabular}

Table 9.1: Magnetic polarisability values and $\chi_{\text {dof }}^{2}$ values from the fits of the energy shift to the field strength for the neutron in units of $\mathrm{fm}^{3} \times 10^{-4}$ at each $\kappa$ value comparing the 1 parameter 2 point fits with the 2 parameter 4 point fits.

the spin-difference had a cubic term. With the quartic term included all four points can be fit. We also perform a single parameter quadratic fit to just the first two points. Comparing both gives an important consistancy check since the quartic contribution is quite large. The values of the polarisability from the one and two parameter fits are given in Table 9.1. The table shows that the 1 and 2 parameter fits agree well within errors. This is largely a symptom of the fact that the first point drives the value to such an extent that the later points don't have that much effect so long as they are within a reasonable range where the quartic term can handle them. This gives us greater confidence in our results because the energy shift plots at the smallest field are the most reasonable to fit, so the difficulty in fitting the higher fields has less effect.

Table 9.1 also displays $\chi_{\text {dof }}^{2}$ values which are $\sim 1$ or even below for both 1 and 2 parameter fits except at the lightest quark mass considered where they are a bit larger due to the first point sitting lower than expected.

\subsubsection{Magnetic polarisability as a function of pion mass}

Using the values from the fits of the mass shift to the field strength we can plot values for the magnetic polarisability of the neutron against the pion mass squared. The polarisability is given in units of $\mathrm{fm}^{3} \times 10^{-4}$. Figure 9.11 shows the polarisability as a function of pion mass squared. Included are both the quenched and dynamical results. There is good agreement between the two sets of results. Both are quite constant across the regime explored, with the lightest point appearing somewhat, but not statistically significantly, higher. 

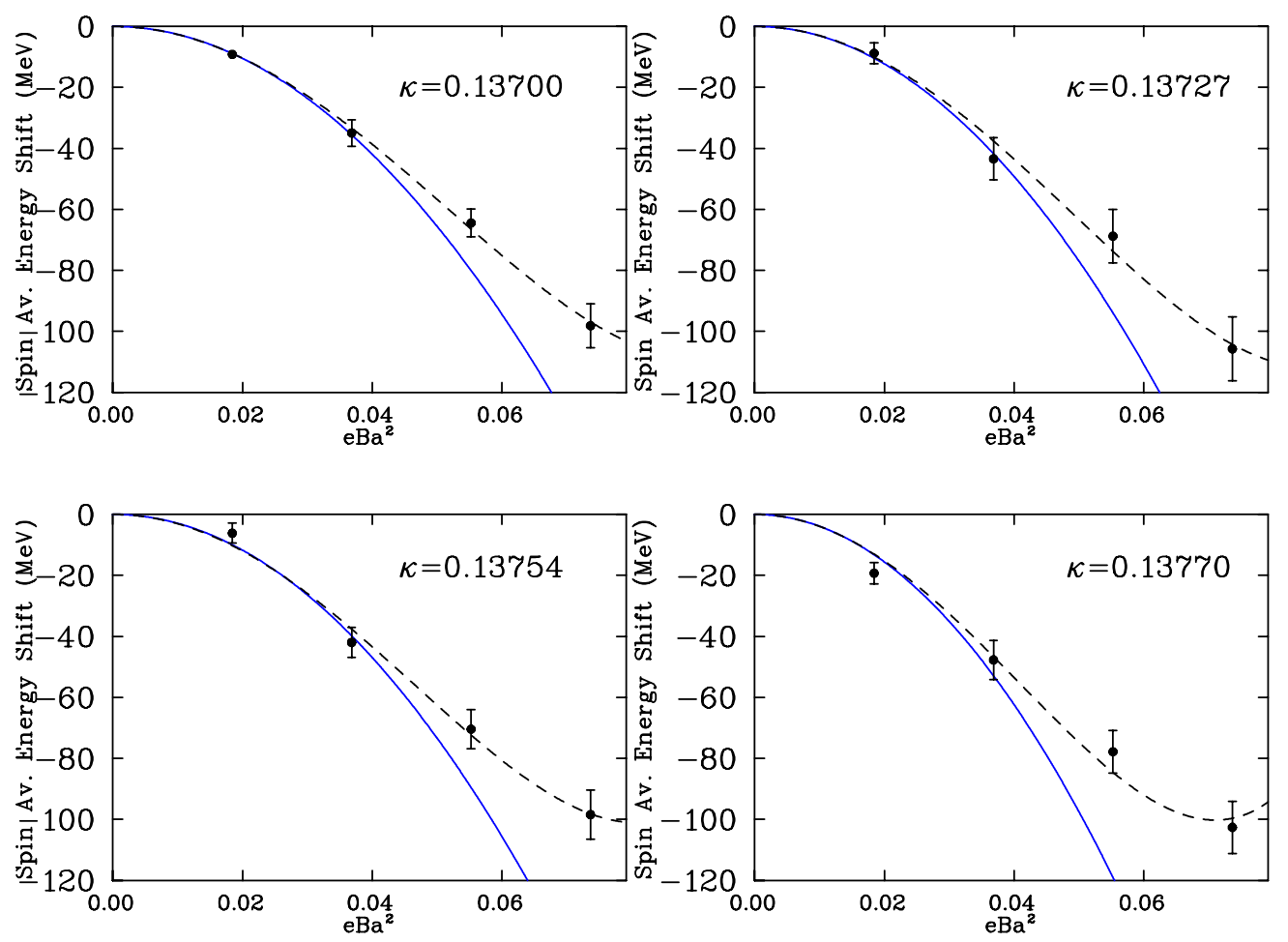

Figure 9.10: Plots of the fits of the spin-averaged mass shift to the magnetic field strength for the neutron. The solid line is a one parameter quadratic fit to just the first two points. The dashed line is a two parameter quadratic plus quartic fit to all four points. 


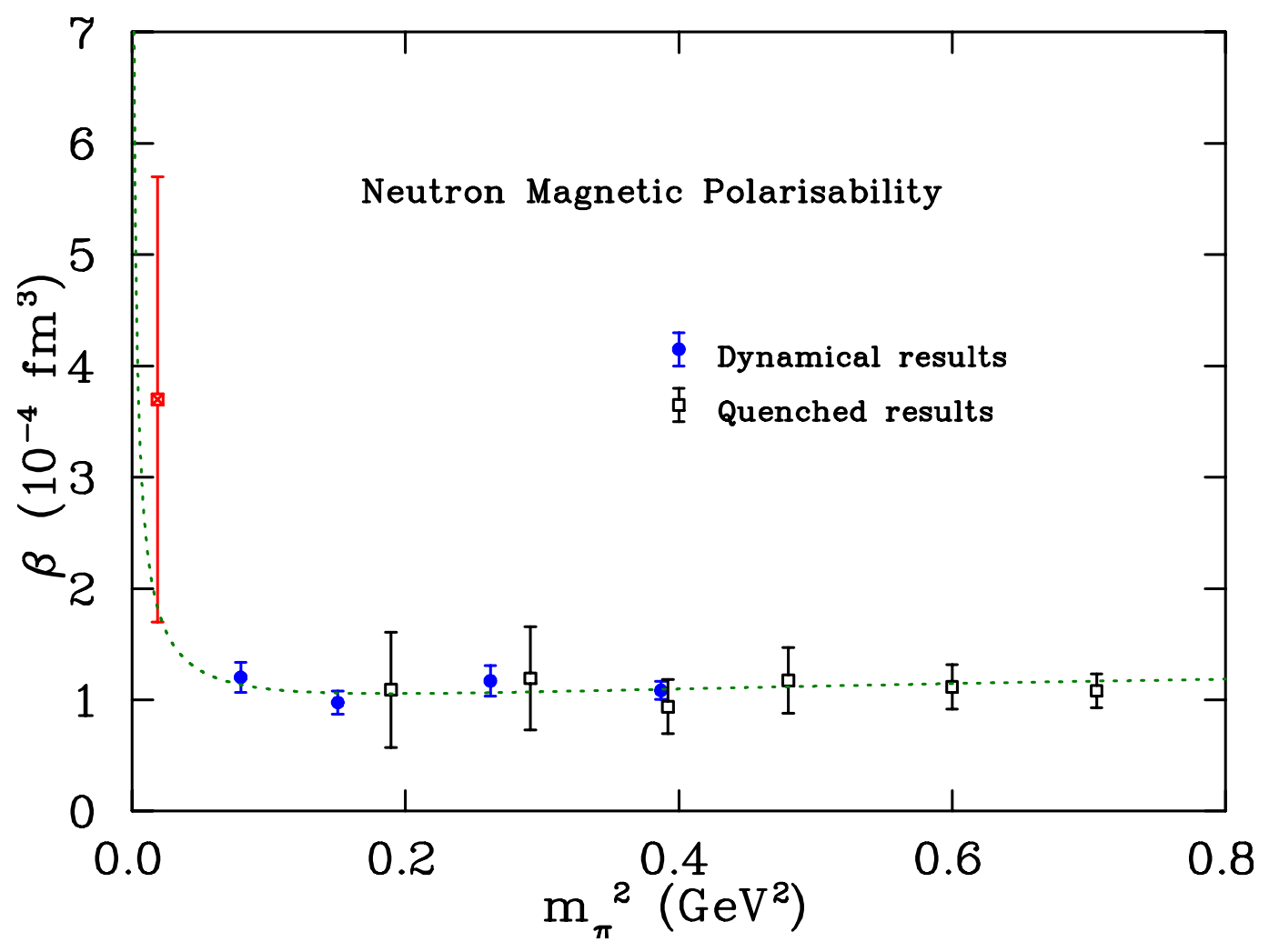

Figure 9.11: The magnetic polarisability of the neutron as a function of pion mass squared. Shown are quenched and dynamical values. The point on the far left is the experimental value. The dashed line represents a chiral fit to the dynamical results.

The point at the far left is the experimental value.

In order to make a prediction at the physical pion mass we performed a chiral extrapolation. The electromagnetic polarisabilities of the nucleon were investigated thoroughly by Bernard, et. al. in the early 90s [51]. Focusing on the leading and next-to-leading terms which are divergent in the chiral limit we have,

$$
\beta_{n}=\frac{C g_{A}^{2}}{8 m_{\pi}}+\frac{C}{\pi}\left[\left(\frac{3\left(1-\kappa_{s}\right) g_{A}^{2}}{M_{N}}-c_{2}\right) \ln \left(m_{\pi}\right)\right],
$$


with,

$$
C=\frac{e^{2}}{96 \pi^{2} F_{\pi}^{2}}=4.36 \cdot 10^{-4} \mathrm{fm}^{2}
$$

and $c_{2}$ estimated via,

$$
c_{2}=\frac{g_{A}^{2}}{2\left(M_{\Delta}-M_{N}\right)}
$$

Here $\kappa_{s}=-0.12$ is the isoscalar anomalous magnetic moment and $g_{A}=1.26$, with $M_{N}$ the physical nucleon mass. This allows us to write an equation for the magnetic polarisability of the neutron as a function of the pion mass, including terms regular for $m_{\pi} \rightarrow 0$,

$$
\beta_{n}=a+\frac{C g_{A}^{2}}{8 m_{\pi}}+\frac{C}{\pi}\left(\frac{3\left(1-\kappa_{s}\right) g_{A}^{2}}{M_{N}}-c_{2}\right) \ln \left(m_{\pi}\right)+b m_{\pi}^{2}
$$

We include a constant and an $m_{\pi}^{2}$ term which are fit freely. The result of this fit is given by the dashed line in Figure 9.11. The line is consistent with the experimental value within its (large) error bars. Our extrapolated value at the physical pion mass is $1.8 \pm 0.2 \times 10^{-4} \mathrm{fm}^{3}$, where the error quoted includes only the statistical uncertainty. Due to the sharp curvature in the chiral region the extrapolated value is quite sensitive to changes in the fit parameters. 


\section{Chapter 10}

\section{Conclusion}

In this work we have shown the effectiveness of the background field method at calculating magnetic properties of hadrons on the lattice. In order to get the most accurate results possible we have performed the first calculation which uses a uniform quantised magnetic field with an exponential phase and periodic boundary conditions.

We have thoroughly investigated issues that need to be understood before performing background field method calculations. We looked at how Landau levels interfere with magnetic polarisability calculations with charged particles. These Landau levels can not be easily taken into account but may be able to be dealt with using a projection onto the lowest Landau energy state.

We described the connection between the origin of the background field and the technique of twisted boundary conditions. Certain choices for the gauge potential that give rise to the background magnetic field cause a constant phase to be included at every site on the lattice. This phase is reminiscent of the type of phase used to implement twisted boundary conditions, however cancellations resulting from the quantisation of the phase mean that it has no effect on physical states. The choice of origin for the field still has an effect on the excited state overlap of a smeared source correlation function and we showed that the best behaviour comes when the quark source and background field source were coincident.

We were able to perform excellent magnetic moment calculations for the proton and neutron. Effective energies were flat and easy to fit and higher order contributions were easily taken into account in the fit to the field. Chiral extrapolations indicated a good approach to the physical values.

Using the variational method we were also able to investigate the mag- 
netic moment of odd-parity nucleon states. We found strong signals for the lowest lying odd-parity states, the $S_{11}^{+}(1535)$ and $S_{11}^{0}(1535)$. Our calculated moments have a projected chiral behaviour which suggests good agreement with predictions made using the chiral constituent quark model. In addition we performed an exploratory calculation of the magnetic moment of the $S_{11}^{+}(1650)$ and $S_{11}^{0}(1650)$. Our values for the two lowest lying odd-parity states support the prediction of an expanded quark model which produced a different sign for the moment of the $S_{11}^{+}(1650)$ than the simplest model.

Calculating the magnetic polarisability of the neutron proved more difficult than the moment. This was due to the fact that it took a lot of Euclidean time evolution for the effective energies to move towards a plateau. We investigated the use of different fermion source and sink smearings to improve the plateau behaviour, including the use of the variational method. There was no significant improvement to be found, but we were still able to construct a plausible criterion for where ground state saturation was achieved and obtain tentative polarisability results which were in good agreement with experiment (within the large error bars of the latter). From chiral perturbation theory the extrapolated value for the magnetic polarisability of the neutron was $1.8 \pm 0.2 \times 10^{-4} \mathrm{fm}^{3}$, where the error quoted includes only the statistical uncertainty.

Overall we have been very successful with our background field method calculations. In the future we hope to improve our results in a number of ways: By moving to lighter quark masses to make the chiral extrapolation easier; using larger correlation matrices to nail down the second odd-parity state; projecting Landau levels to be able to do the proton polarisability; investigating the potential for Landau level effects on the neutron; performing a volume analysis to properly account for finite volume effects. We look forward to presenting this future work. 


\section{Acknowledgements}

We are grateful for the generosity of the PACS-CS collaboration for providing the gauge configurations used in this study. The contributions of the ILDG in making the configurations accessible is also appreciated. This research was undertaken with the assistance of resources at the NCI National Facility in Canberra, Australia, and the iVEC facilities at the University of Western Australia. These resources were provided through the National Computational Merit Allocation Scheme, supported by the Australian Government. This research is supported by the Australian Research Council. 


\section{Bibliography}

[1] R. Gupta, "Introduction to lattice QCD: Course," pp. 83-219, 1997.

[2] S. Chatrchyan et al., "Observation of a new boson at a mass of $125 \mathrm{GeV}$ with the CMS experiment at the LHC," Physics Letters B, vol. 716, no. 1 , pp. $30-61,2012$.

[3] G. Aad et al., "Observation of a new particle in the search for the Standard Model Higgs boson with the ATLAS detector at the LHC," Physics Letters B, vol. 716, no. 1, pp. 1 - 29, 2012.

[4] D. Griffiths, Introduction to Elementary Particles. 2008.

[5] P. Dirac, The Principles of Quantum Mechanics (International Series of Monographs on Physics). 1982.

[6] K. G. Wilson, "Confinement of Quarks," Phys.Rev., vol. D10, pp. 24452459, 1974.

[7] B. Sheikholeslami and R. Wohlert, "Improved Continuum Limit Lattice Action for QCD with Wilson Fermions," Nucl.Phys., vol. B259, p. 572, 1985 .

[8] S. Aoki et al., "Nonperturbative $\mathrm{O}(\mathrm{a})$ improvement of the Wilson quark action with the RG-improved gauge action using the Schrodinger functional method," Phys.Rev., vol. D73, p. 034501, 2006.

[9] C. Bernard, T. Draper, and K. Olynyk, "Lattice QCD calculation of some baryon magnetic moments," Phys.Rev.Lett., vol. 49, p. 1076, 1982.

[10] J. Smit and J. Vink, "Remnants of the Index Theorem on the Lattice," Nucl.Phys., vol. B286, p. 485, 1987. 
[11] H. Fiebig, W. Wilcox, and R. Woloshyn, "A study of hadron electric polarizability in quenched lattice QCD," Nucl.Phys., vol. B324, p. 47, 1989 .

[12] H. Rubinstein, S. Solomon, and T. Wittlich, "Dependence of lattice hadron masses on external magnetic fields," Nucl.Phys., vol. B457, pp. 577-593, 1995.

[13] M. Burkardt, D. Leinweber, and X. Jin, "Background field formalism in quantum systems," Phys.Lett., vol. B385, pp. 52-56, 1996.

[14] L. Zhou, F. Lee, W. Wilcox, and J. Christensen, "Magnetic polarizability of hadrons from lattice QCD," Nucl.Phys.Proc.Suppl., vol. 119, pp. 272274, 2003.

[15] F. Lee, R. Kelly, L. Zhou, and W. Wilcox, "Baryon magnetic moments in the background field method," Phys.Lett., vol. B627, pp. 71-76, 2005.

[16] F. Lee, L. Zhou, W. Wilcox, and J. Christensen, "Magnetic Polarizability of hadrons from lattice QCD in the background field method," Phys.Rev., vol. D73, p. 034503, 2006.

[17] W. Detmold, B. Tiburzi, and A. Walker-Loud, "Electromagnetic and spin polarisabilities in lattice QCD," Phys.Rev., vol. D73, p. 114505, 2006 .

[18] M. Engelhardt, "Neutron electric polarizability from unquenched lattice QCD using the background field approach," Phys.Rev., vol. D76, p. 114502, 2007.

[19] F. Lee, S. Moerschbacher, and W. Wilcox, "Magnetic moments of vector, axial, and tensor mesons in lattice QCD," Phys.Rev., vol. D78, p. 094502, 2008.

[20] A. Alexandru and F. X. Lee, "The Background field method on the lattice," PoS, vol. LATTICE2008, p. 145, 2008.

[21] B. Tiburzi and S. Vayl, "Charged Hadron Properties from Lattice QCD in Magnetic Fields," Phys.Rev., vol. D87, p. 054507, 2013.

[22] S. Aoki, et al. (PACS-CS Collaboration), "2+1 Flavor Lattice QCD toward the Physical Point," Phys.Rev., vol. D79, p. 034503, 2009. 
[23] M. G. Beckett, et al., "Building the International Lattice Data Grid," Comput. Phys. Commun., vol. 182, pp. 1208-1214, 2001.

[24] R. Sommer, "A New way to set the energy scale in lattice gauge theories and its applications to the static force and alpha-s in SU(2) Yang-Mills theory," Nucl.Phys., vol. B411, pp. 839-854, 1994.

[25] W. Freeman, A. Alexandru, F. Lee, and M. Lujan, "Sea Contributions to Hadron Electric Polarizabilities through Reweighting," PoS International Lattice Conference 2012, 2012.

[26] C. Itzykson and J. B. Zuber, Quantum Field Theory. 1980.

[27] J. Beringer, et al. (Particle Data Group), "Review of Particle Physics (RPP)," Phys.Rev., vol. D86, p. 010001, 2012.

[28] P. F. Bedaque, "Aharonov-Bohm effect and nucleon nucleon phase shifts on the lattice," Phys.Lett., vol. B593, pp. 82-88, 2004.

[29] G. de Divitiis, R. Petronzio, and N. Tantalo, "On the discretization of physical momenta in lattice QCD," Phys.Lett., vol. B595, pp. 408-413, 2004.

[30] A. Alexandru and F. Lee, "Hadron electric polarizability - finite volume corrections," PoS, vol. LATTICE2010, p. 131, 2010.

[31] D. Leinweber, R. Woloshyn, and T. Draper, "Electromagnetic structure of octet baryons," Phys.Rev., vol. D43, pp. 1659-1678, 1991.

[32] S. Boinepalli, D. B. Leinweber, A. G. Williams, J. M. Zanotti, and J. B. Zhang, "Precision Electromagnetic Structure of Octet Baryons in the Chiral Regime," Phys.Rev., vol. D74, p. 093005, 2006.

[33] S. Collins, M. Gockeler, P. Hagler, R. Horsley, Y. Nakamura, et al., "Dirac and Pauli Form factors from lattice QCD," Phys.Rev., vol. D84, p. 074507, 2011.

[34] G. Morpurgo, "Smallness of gluon coupling to constituent quarks in baryons and the validity of the nonrelativistic quark model," Phys.Rev., vol. D46, pp. 4068-4075, 1992. 
[35] D. B. Leinweber, A. W. Thomas, and R. D. Young, "Chiral symmetry and the intrinsic structure of the nucleon," Phys.Rev.Lett., vol. 86, pp. 5011-5014, 2001.

[36] J. Hall, D. Leinweber, and R. Young, "Chiral extrapolations for nucleon magnetic moments," Phys.Rev., vol. D85, p. 094502, 2012.

[37] D. B. Leinweber and A. W. Thomas, "A Lattice QCD analysis of the strangeness magnetic moment of the nucleon," Phys.Rev., vol. D62, p. $074505,2000$.

[38] F. X. Lee and A. Alexandru, "Magnetic Moments of Negative-Parity Baryons from Lattice QCD," PoS, vol. LATTICE2010, p. 148, 2010.

[39] M. S. Mahbub, W. Kamleh, D. B. Leinweber, P. J. Moran, and A. G. Williams, "Roper Resonance in 2+1 Flavor QCD," Phys.Lett., vol. B707, pp. 389-393, 2012.

[40] M. Mahbub, W. Kamleh, D. Leinweber, P. Moran, and A. Williams, "Exploring excited states of the nucleon in $2+1$ flavor lattice QCD," AIP Conf.Proc., vol. 1432, pp. 261-264, 2012.

[41] B. J. Menadue, W. Kamleh, D. B. Leinweber, and M. S. Mahbub, "Isolating the $\Lambda(1405)$ in Lattice QCD," Phys.Rev.Lett., vol. 108, p. 112001, 2012 .

[42] M. S. Mahbub, W. Kamleh, D. B. Leinweber, P. J. Moran, and A. G. Williams, "Low-lying Odd-parity States of the Nucleon in Lattice QCD," Phys.Rev., vol. D87, p. 011501, 2013.

[43] M. S. Mahbub, W. Kamleh, D. B. Leinweber, P. J. Moran, and A. G. Williams, "Structure and Flow of the Nucleon Eigenstates in Lattice QCD," 2013.

[44] W.-T. Chiang, S.-N. Yang, M. Vanderhaeghen, and D. Drechsel, "Magnetic moment of the $S_{11}(1535)$ resonance," Nucl.Phys., vol. A721, pp. 731-734, 2003.

[45] N. Sharma, A. Martinez Torres, K. Khemchandani, and H. Dahiya, "Magnetic Moments of the $S_{11}(1535)$ and $S_{11}(1650)$ Resonances, and Low-Lying Negative Parity Baryons," Eur.Phys.J., vol. A49, p. 11, 2013. 
[46] F. Federspiel, R. Eisenstein, M. Lucas, B. MacGibbon, K. Mellendorf, et al., "The Proton Compton effect: A Measurement of the electric and magnetic polarizabilities of the proton," Phys.Rev.Lett., vol. 67, pp. 1511-1514, 1991.

[47] A. Zieger, R. Van de Vyver, D. Christmann, A. De Graeve, C. Van den Abeele, et al., "180-degrees Compton scattering by the proton below the pion threshold," Phys.Lett., vol. B278, pp. 34-38, 1992.

[48] V. Olmos de Leon, F. Wissmann, P. Achenbach, J. Ahrens, H. Arends, et al., "Low-energy Compton scattering and the polarizabilities of the proton," Eur.Phys.J., vol. A10, pp. 207-215, 2001.

[49] K. Kossert, M. Camen, F. Wissmann, J. Ahrens, J. Annand, et al., "Quasifree Compton scattering and the polarizabilities of the neutron," Eur.Phys.J., vol. A16, pp. 259-273, 2003.

[50] M. Lundin, J. Adler, T. Glebe, K. Fissum, K. Hansen, et al., "Compton scattering from the deuteron and neutron polarizabilities," Phys.Rev.Lett., vol. 90, p. 192501, 2003.

[51] V. Bernard, N. Kaiser, U. G. Meissner, and A. Schmidt, "Aspects of nucleon Compton scattering," Z.Phys., vol. A348, p. 317, 1994. 


\section{Appendix A}

\section{Quenched Results}

\section{A.1 Simulation details}

Our quenched calculations were performed on $32^{3} \times 40$ lattices using a Symanzik improved gauge action. We used a FLIC fermion action with $\beta=4.52$ and seven values of the hopping parameter $\kappa_{\mathrm{ud}}$. These give us a lattice spacing $a=0.128 \mathrm{fm}$ and pion masses $m_{\pi}=840,775,693,626,540,435$ and 275 $\mathrm{MeV}$. We use periodic boundary conditions in the spatial dimensions and a fixed boundary condition in time.

For each mass we had an ensemble of 192 configurations. We used four values of the dimensionless magnetic field value $e B a^{2}=2 \pi n / n_{x} n_{y}=+0.0061$, $-0.0123,+0.0245,-0.0492$, corresponding to $n=1,-2,4,-8$. This is the field experienced by the $d$ quark, with the $u$ quark experiencing -2 times these values for a field $B$ experienced by a proton of -3 times these values. By choosing fields related to the previous field by a factor of -2 , the same factor relating the quark charges, we require only five propagators (not including zero field) to get both quarks in four non-zero magnetic field strengths. The propagators were source smeared with 40 sweeps of Gaussian smearing with a smearing coefficient of 0.7 .

\section{A.2 Magnetic moment}

The calculation of the quenched magnetic moment and magnetic polarisability results follows the same method as for the dynamical results. We 
construct a spin-difference effective energy using the form,

$$
\Delta E(B)=\frac{1}{2}\left(\frac{G_{\uparrow}(B, t)}{G_{\uparrow}(0, t)} \frac{G_{\downarrow}(0, t)}{G_{\downarrow}(B, t)}\right)_{f i t} .
$$

Figures A.1-A.7 show the spin-difference effective energy shifts for the proton and neutron at all seven quark masses. We see in each plot the expected progression to larger energy shifts as the strength of the field increases. Here the field doubles each time unlike the linear progression we used for the dynamical results, so although the gap between the curves gets larger at higher field strengths the shift due to the field is getting proportionally smaller, just as in the dynamical case. There appears to be more excited state contribution than we saw with the dynamical results, likely due to the 40 sweeps compared to 100 sweeps for the dynamical results which improved the ground state overlap.

The proton and neutron are similar in the shapes of their curves, with the neutron taking longer to reach a plateau at the larger field strengths, but also maintaining signal for longer. As expected the signal gets more and more noisy as we move to lighter quark masses, however the three smallest field strengths are reasonable in each case. The largest field strength shows significant curvature at early times and does not reach a good plateau at the lighter quark masses. This is the reason we switched our field strengths from $1,-2,4,-8$ to $1,-2,3,4$ when we performed our dynamical calculation. Although it required an extra propagator to be calculated the data point for the 3rd field strength was much more useful than the overly large one here.

We determined fitting windows based on the same criteria as described in Section 8.4.1, looking for the earliest possible window with a reasonable $\chi_{\text {dof }}^{2}$ value. Although there was no difficulty in finding an acceptable window for each mass and field, the late plateaus at high field strengths and increase of noise at light quark masses meant that we could not pick a single window to apply across every fit. Despite this we tried to keep them as consistent as possible and since the first two field strengths which are the most important to the moment value are mostly flat, we are confident that no significant systematic error is introduced by the choice of fit windows.

Figures A.8 and A.9 show the fits of the spin-difference energy shift against the field strength. As with the dynamical results we had to include a cubic term in order to fit the third and fourth field strengths with an acceptable $\chi^{2}$ value. Again we performed a single parameter fit to just the 


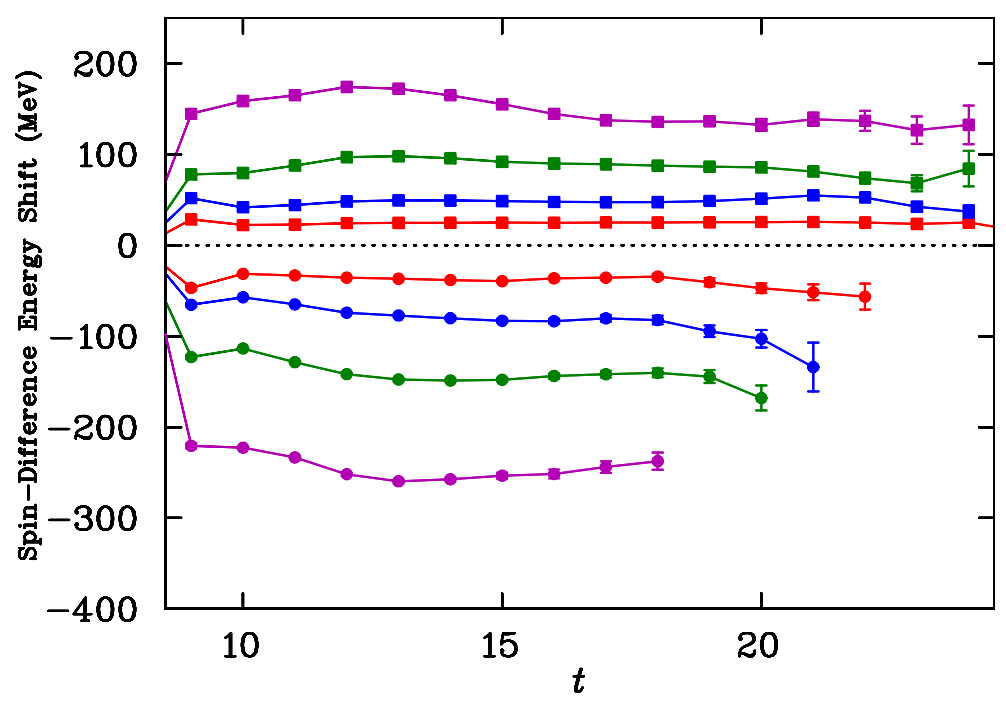

Figure A.1: Quenched QCD energy shifts for the difference of spin-up and spin-down at $m_{\pi}=840 \mathrm{MeV}$ for all field strengths. The top (positive) curves are for the neutron and the bottom (negative) curves are for the proton.

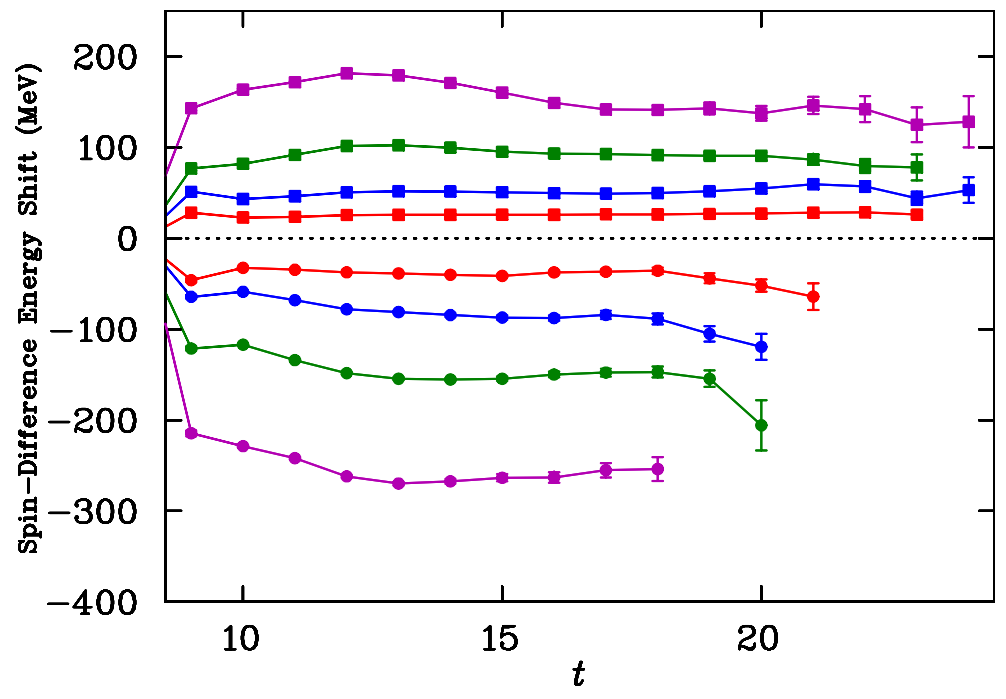

Figure A.2: Quenched QCD mass shift for the difference of spin-up and spindown at $m_{\pi}=775 \mathrm{MeV}$ for all field strengths. The top (positive) curves are for the neutron and the bottom (negative) curves are for the proton. 


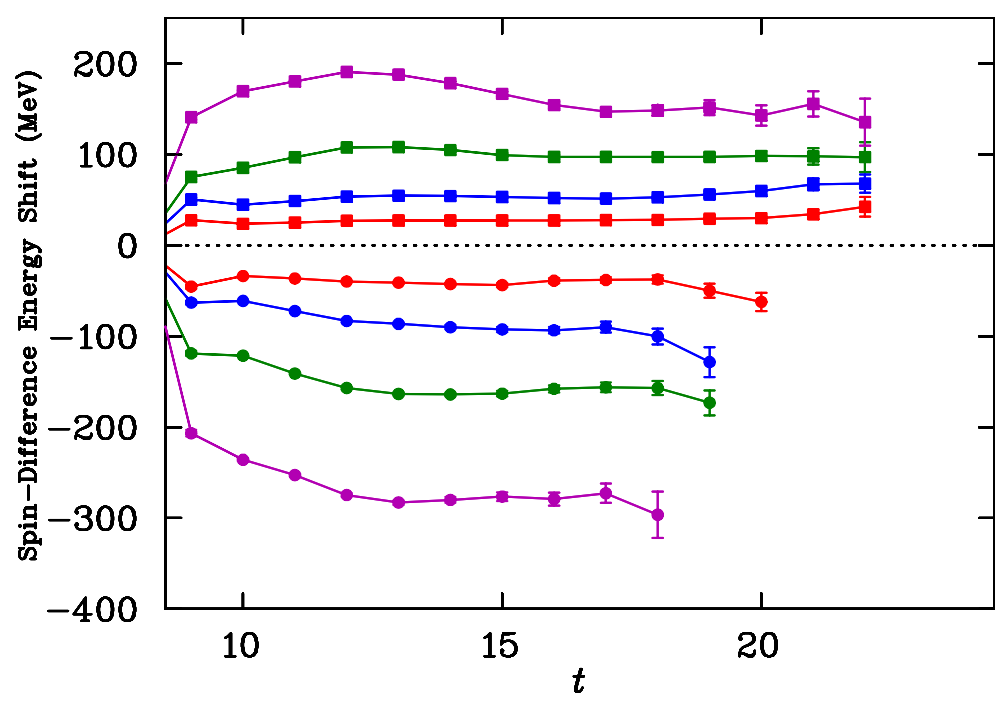

Figure A.3: Quenched QCD effective energies for the difference of spin-up and spin-down at $m_{\pi}=693 \mathrm{MeV}$ for all field strengths. The top (positive) curves are for the neutron and the bottom (negative) curves are for the proton.

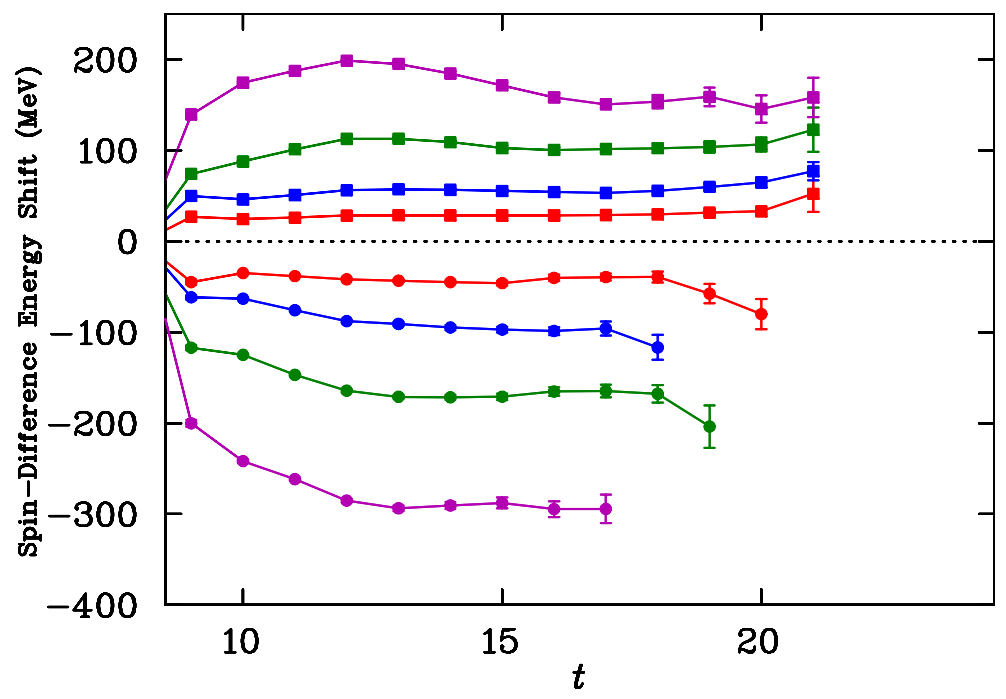

Figure A.4: Quenched QCD effective energies for the difference of spin-up and spin-down at $m_{\pi}=626 \mathrm{MeV}$ for all field strengths. The top (positive) curves are for the neutron and the bottom (negative) curves are for the proton. 


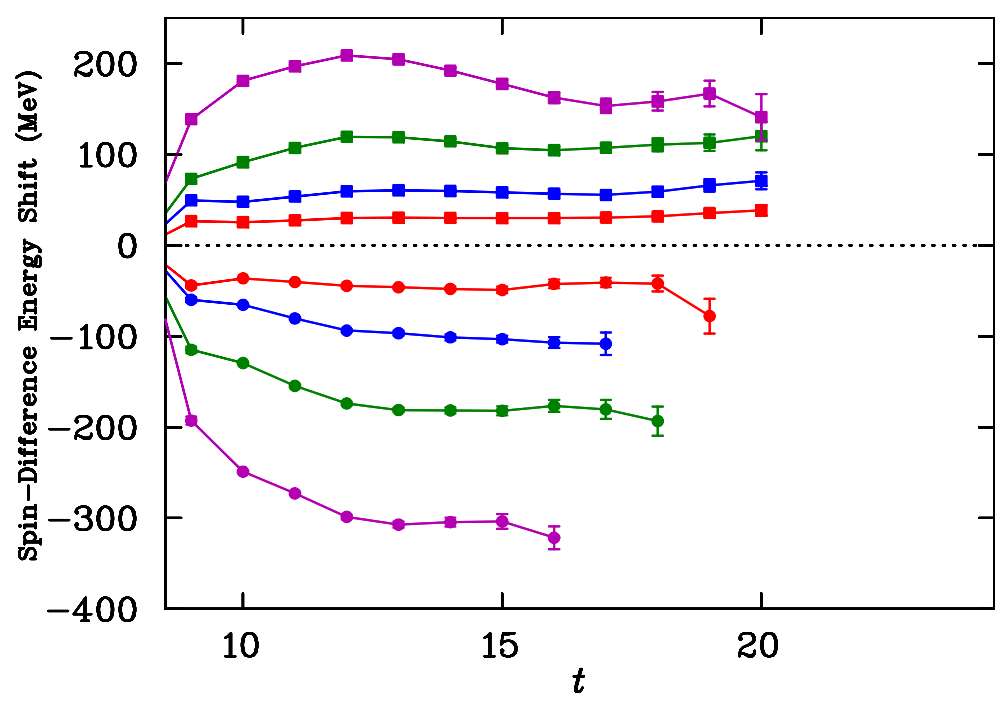

Figure A.5: Quenched QCD effective energies for the difference of spin-up and spin-down at $m_{\pi}=540 \mathrm{MeV}$ for all field strengths. The top (positive) curves are for the neutron and the bottom (negative) curves are for the proton.

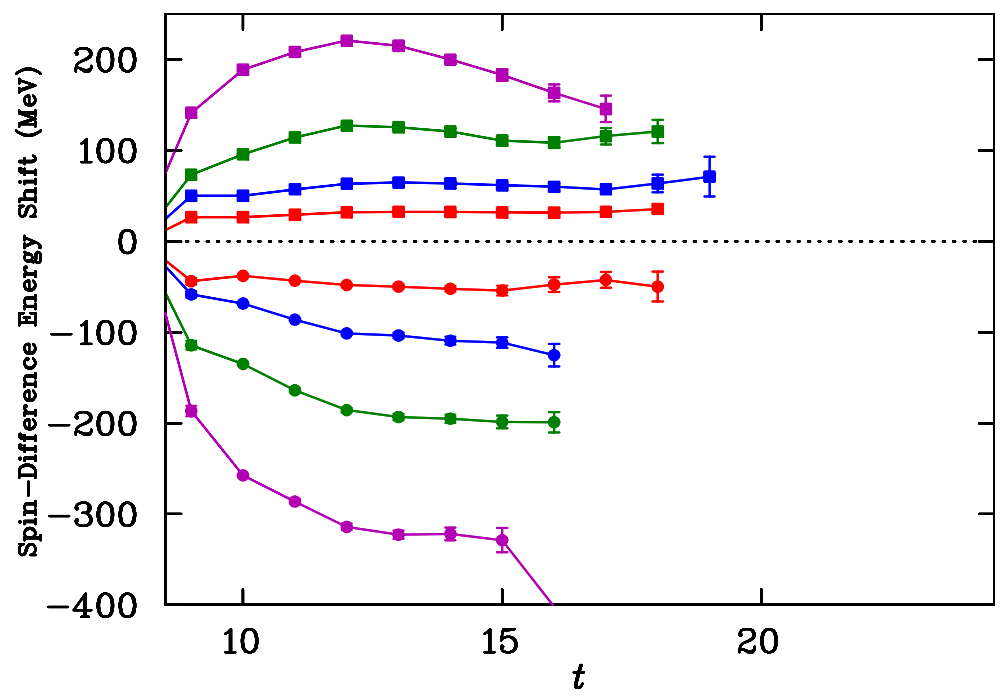

Figure A.6: Quenched QCD effective energies for the difference of spin-up and spin-down at $m_{\pi}=435 \mathrm{MeV}$ for all field strengths. The top (positive) curves are for the neutron and the bottom (negative) curves are for the proton. 


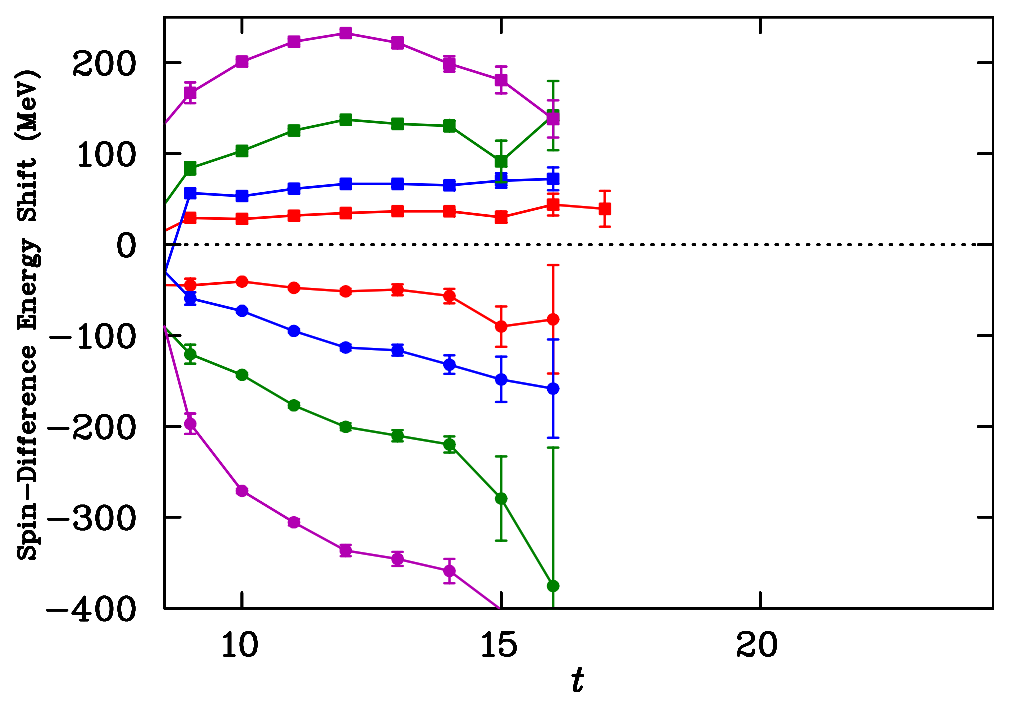

Figure A.7: Quenched QCD effective energies for the difference of spin-up and spin-down at $m_{\pi}=275 \mathrm{MeV}$ for all field strengths. The top (positive) curves are for the neutron and the bottom (negative) curves are for the proton.

first two points for consistency and found that the two results agreed within errors.

Figures A.10 and A.11 show the magnetic moment values from the quenched configurations as a function of pion mass squared. They are compared with values from another quenched calculation which used the form factor method [32]. For the proton the two sets of values agree quite well throughout the range of pion masses. The neutron values from our background field calculation are somewhat larger than the form factor ones, although the large errors on the form factor values means they agree in the light quark mass region. 

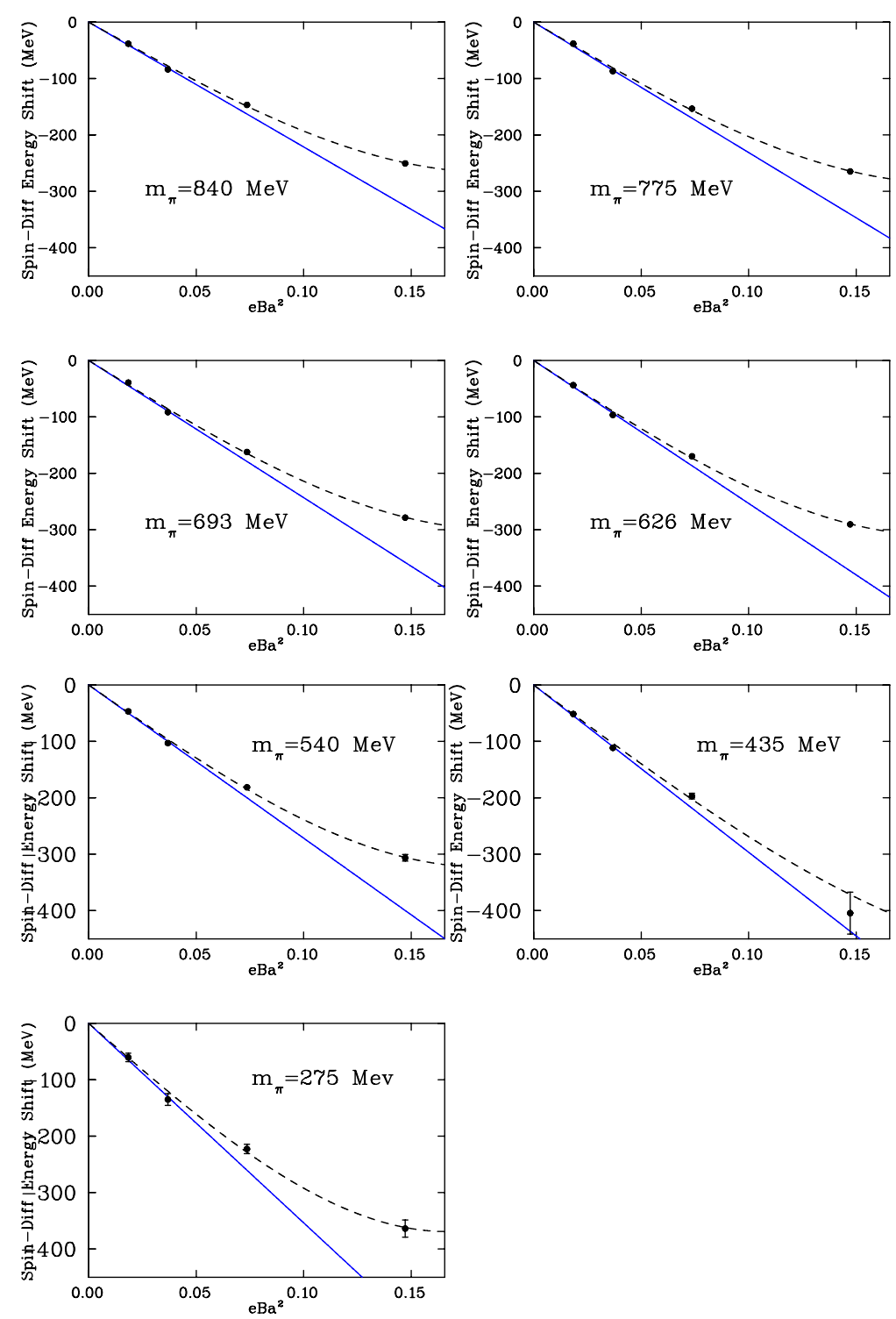

Figure A.8: Fits of the quenched proton spin-difference effective energy shift against the field at each quark mass. The solid line is a 1 parameter fit to the first two points, the dashed line is a 2 parameter fit to all 4 points. 

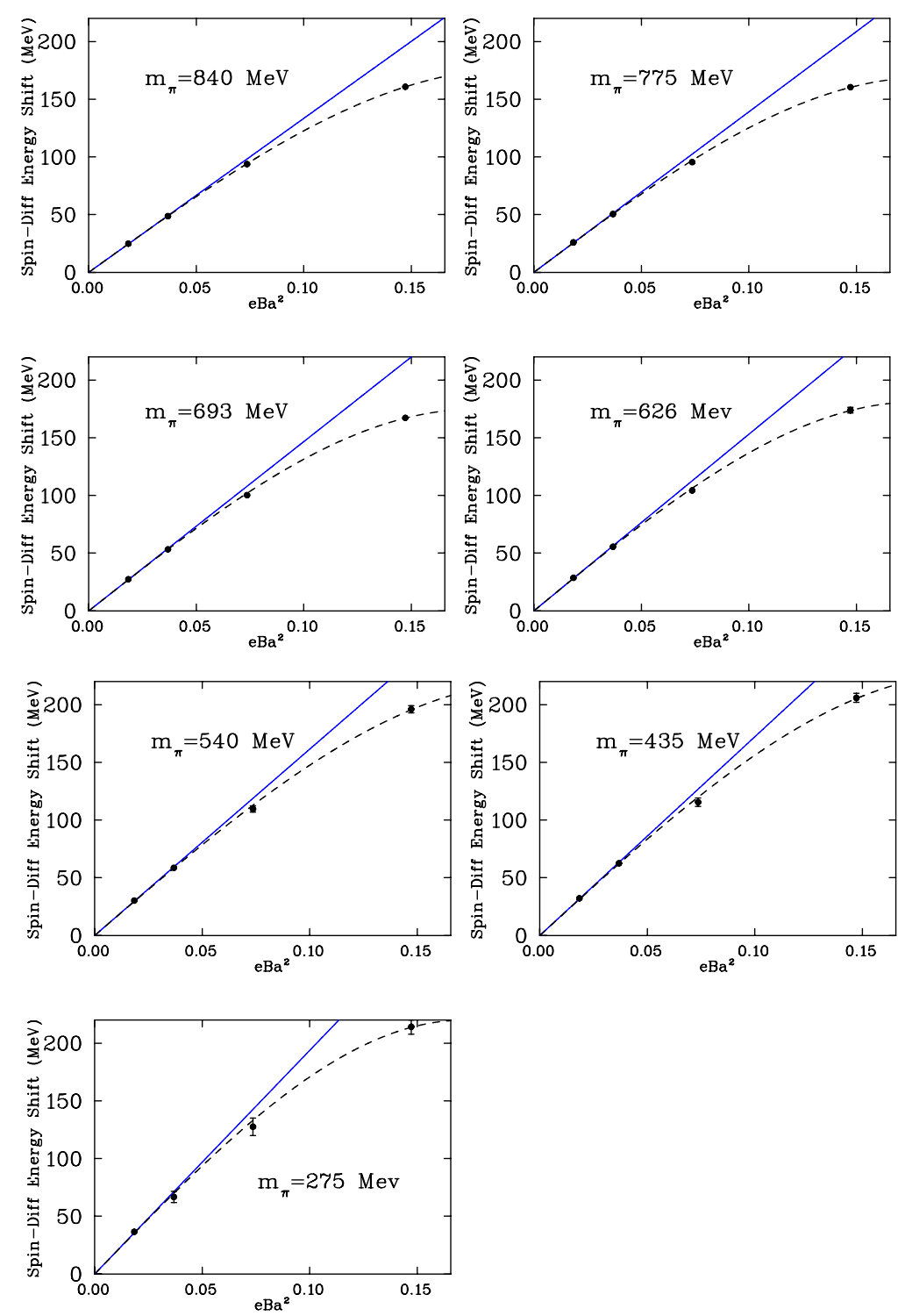

Figure A.9: Fits of the quenched neutron spin-difference effective energy shift against the field at each quark mass. The solid line is a 1 parameter fit to the first two points, the dashed line is a 2 parameter fit to all 4 points. 


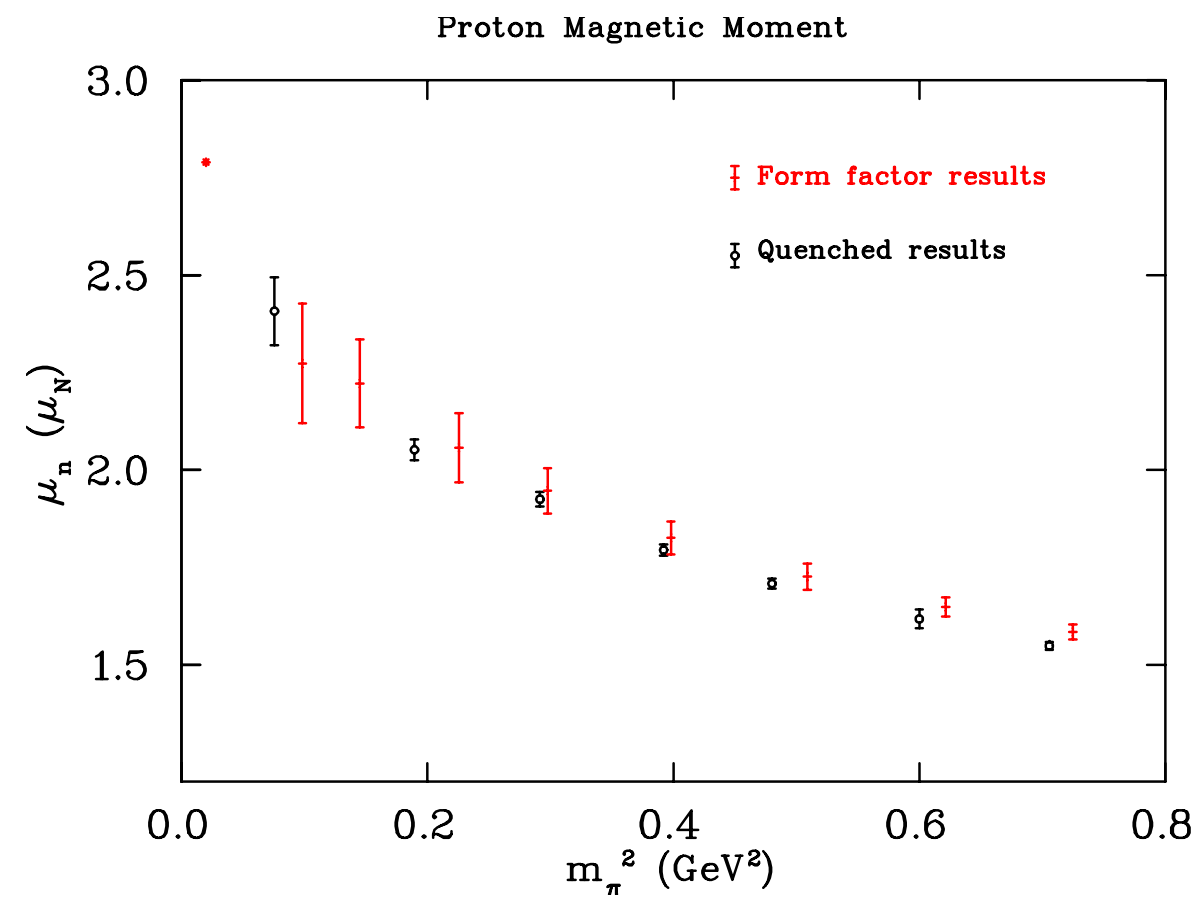

Figure A.10: The magnetic moment of the proton as a function of pion mass squared. The left-most point is the experimental value. Form factor results taken from Ref. [32]. 


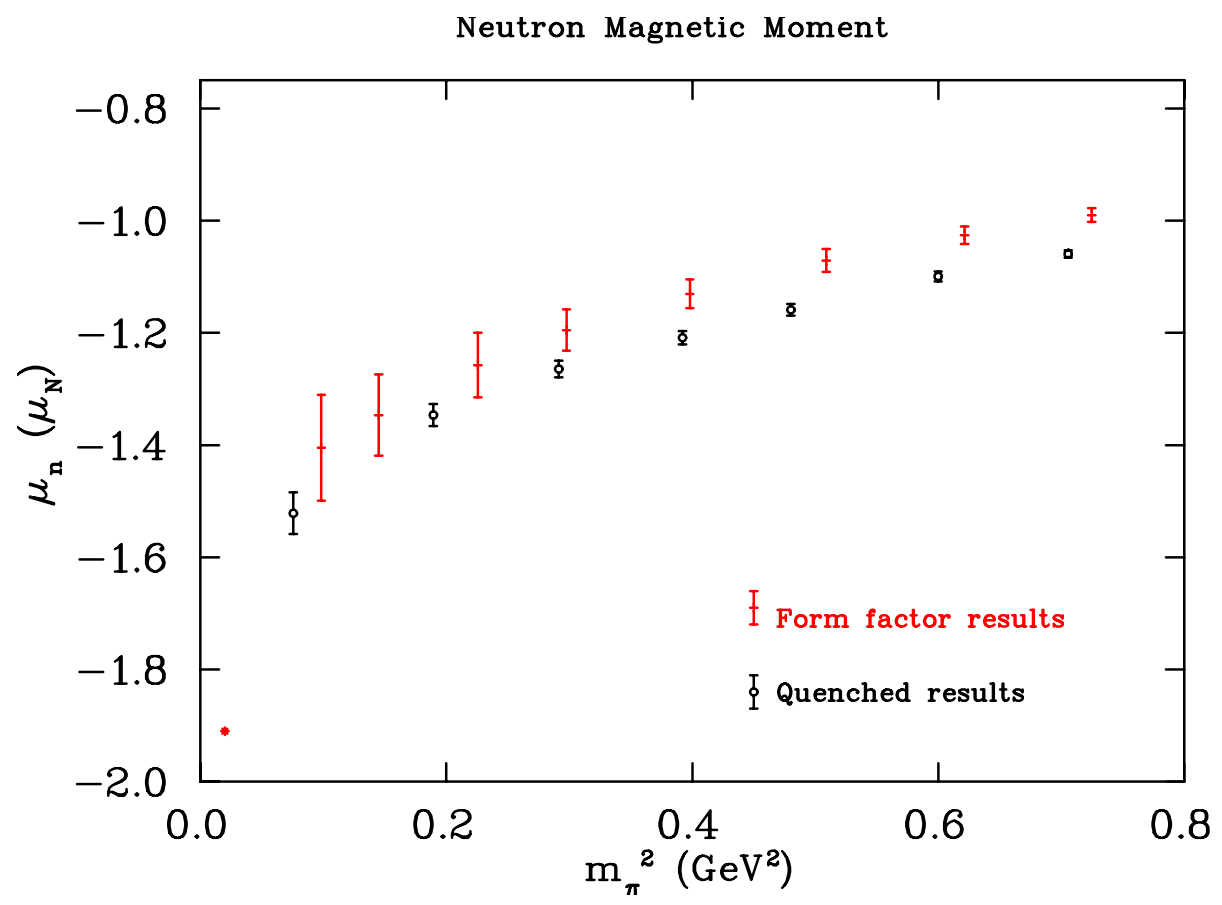

Figure A.11: The magnetic moment of the neutron as a function of pion mass squared. The left-most point is the experimental value. Form factor results taken from Ref. [32]. 


\section{A.3 Magnetic polarisability}

Figures A.12 to A.18 show effective energy plots for the shift due to the magnetic field averaged over spin-up and spin-down neutrons. These follow the same general trend as the dynamical versions, starting as a small shift and growing with Euclidean time before becoming overcome with noise. The curves do not all start at a point like the dynamical ones did because the smaller amount of source smearing used on the quenched propagators has resulted in more excited state contamination at early times. The smallest field strength plateaus fairly well but is consistent with zero at almost every mass. This is because the smallest field strength here is very small and the ensemble is not as large as our dynamical ensemble, leading to larger errors. The lightest mass is so noisy that the plots do not retain signal for long enough to extract any meaningful results, therefore we do not include them in our polarisability results.

Figure A.19 shows the fits of the spin-averaged energy shift to the magnetic field strength. We performed a two parameter fit with quadratic and quartic terms at all four points and a purely quadratic fit to the first three points. The quadratic coefficients of the two fits (which give the polarisability) agreed within errors. We could include the third point in the one parameter fit because it has larger errors than we saw in the dynamical results so the higher order contributions are not apparent at that field strength. The final quenched polarisability results can be seen in Section 9.3.4 in Figure 9.11. 


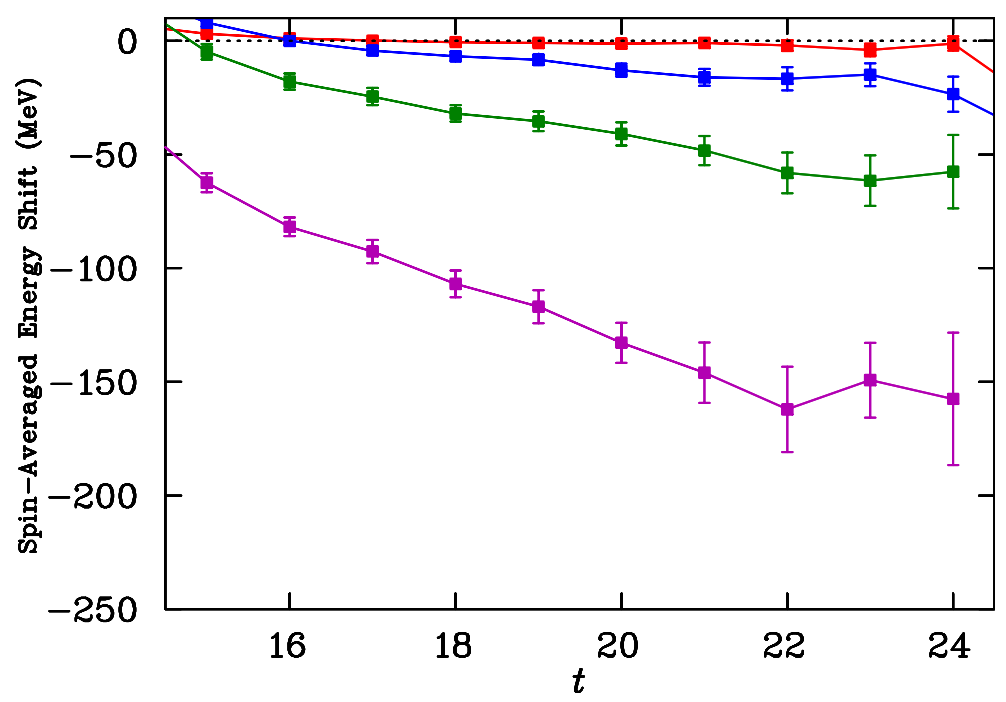

Figure A.12: Quenched QCD energy shifts for the average of spin-up and spin-down at $m_{\pi}=840 \mathrm{MeV}$ for all field strengths.

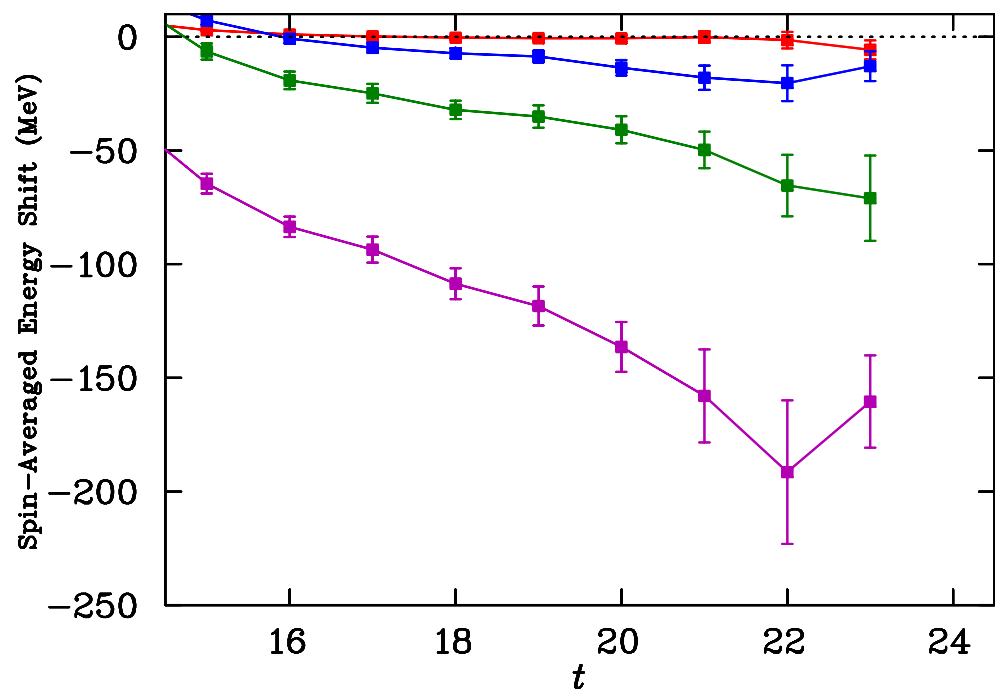

Figure A.13: Quenched QCD energy shifts for the average of spin-up and spin-down at $m_{\pi}=775 \mathrm{MeV}$ for all field strengths. 


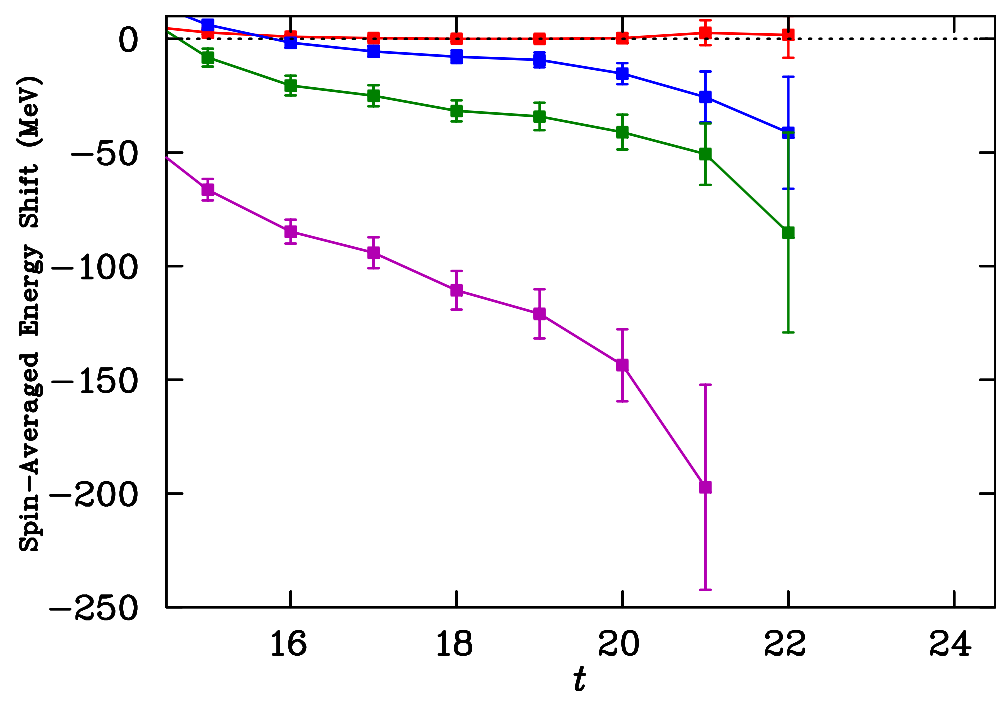

Figure A.14: Quenched QCD energy shifts for the average of spin-up and spin-down at $m_{\pi}=693 \mathrm{MeV}$ for all field strengths.

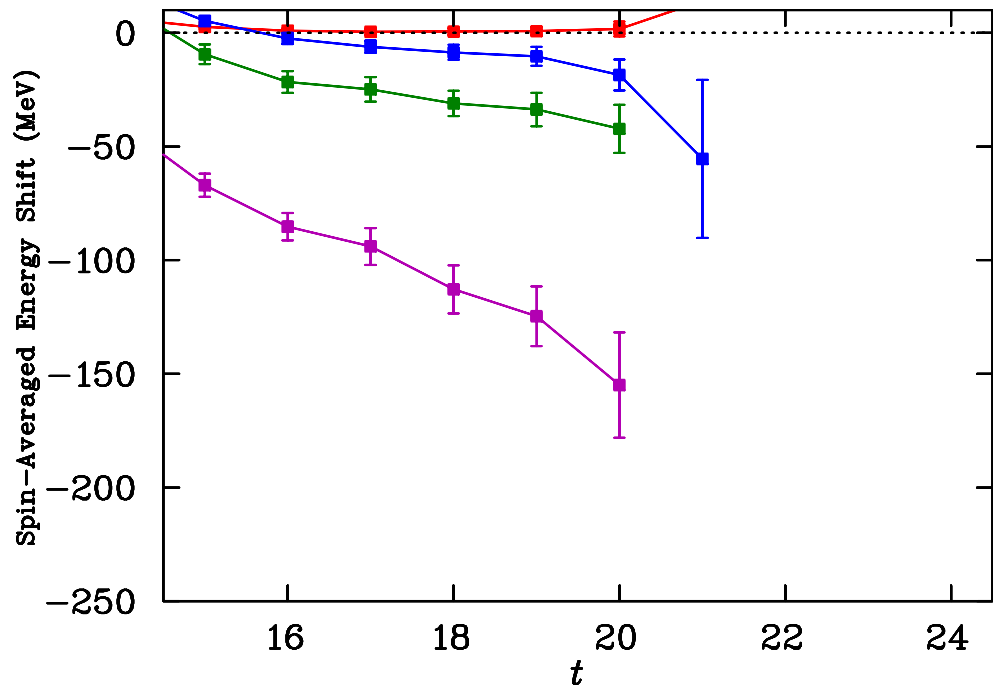

Figure A.15: Quenched QCD energy shifts for the average of spin-up and spin-down at $m_{\pi}=626 \mathrm{MeV}$ for all field strengths. 


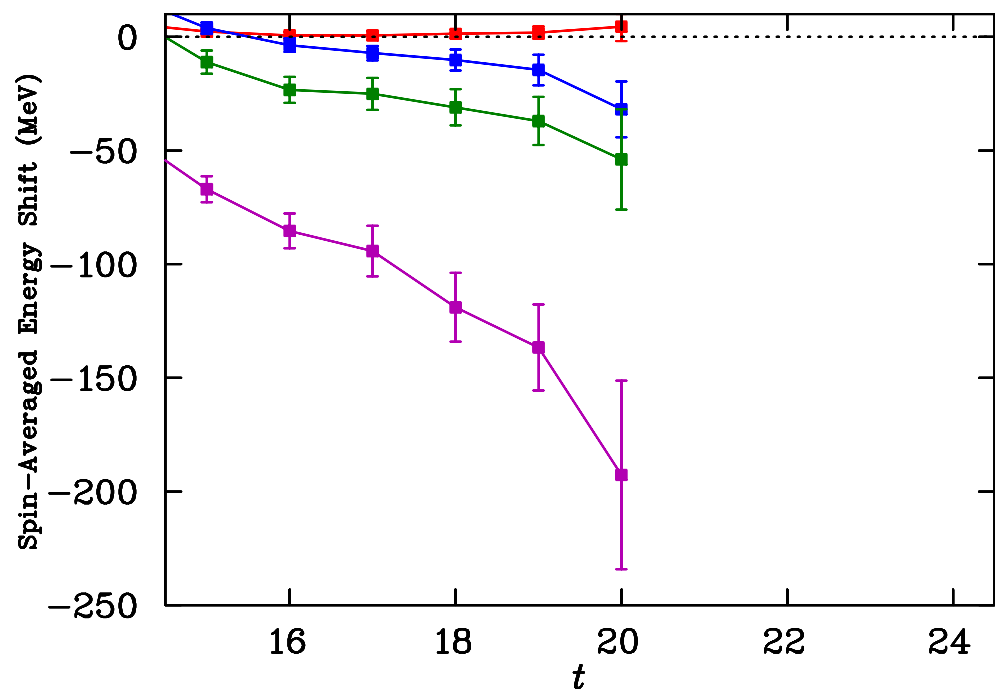

Figure A.16: Quenched QCD energy shifts for the average of spin-up and spin-down at $m_{\pi}=540 \mathrm{MeV}$ for all field strengths.

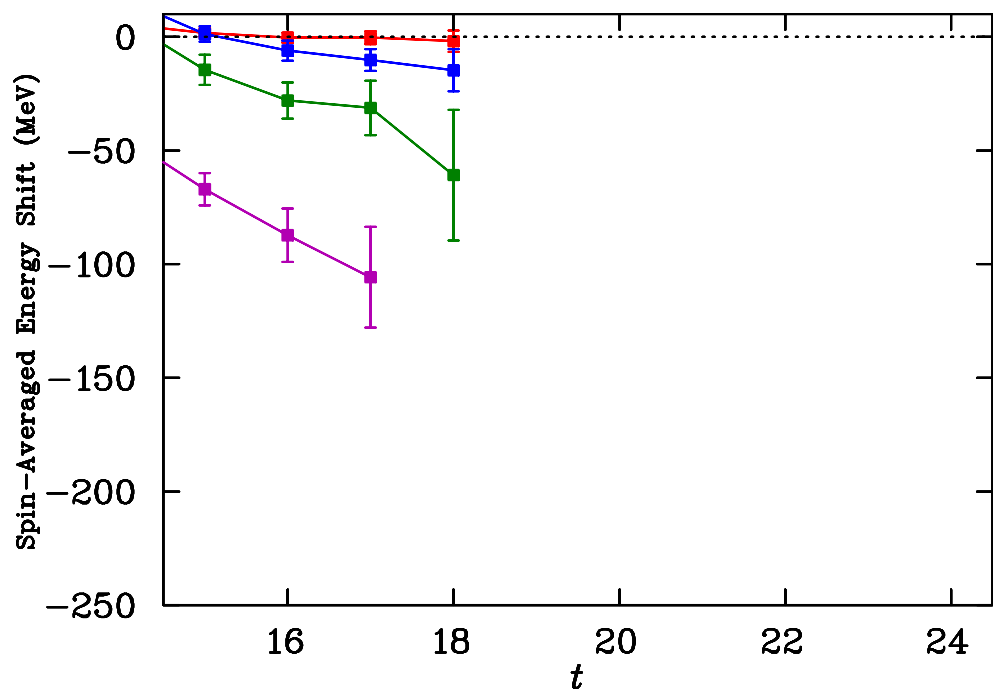

Figure A.17: Quenched QCD energy shifts for the average of spin-up and spin-down at $m_{\pi}=435 \mathrm{MeV}$ for all field strengths. 


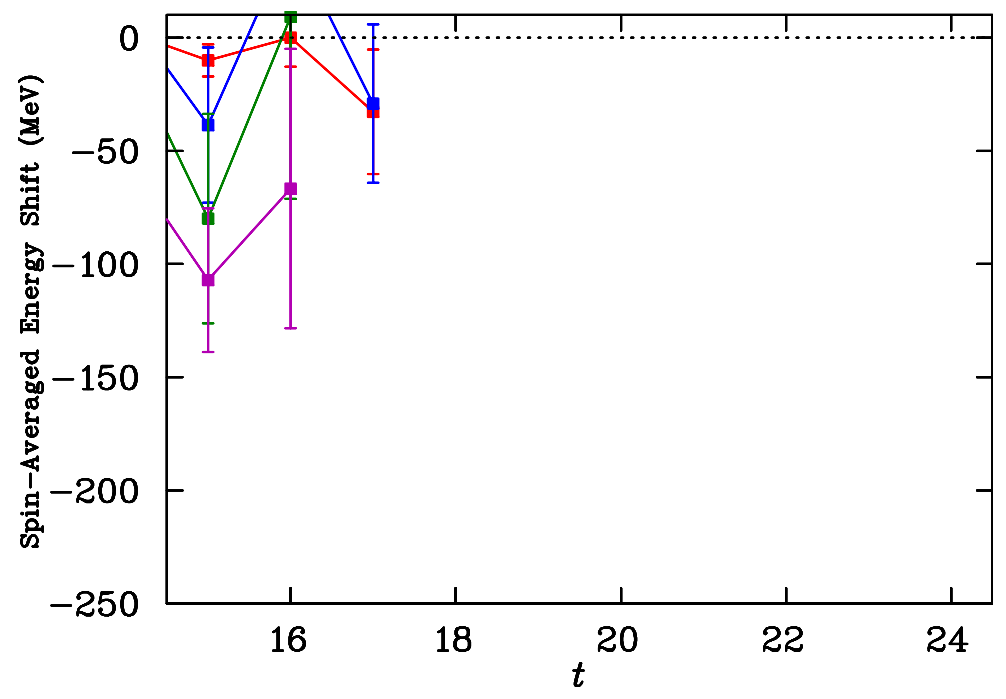

Figure A.18: Quenched QCD energy shifts for the average of spin-up and spin-down at $m_{\pi}=275 \mathrm{MeV}$ for all field strengths. 

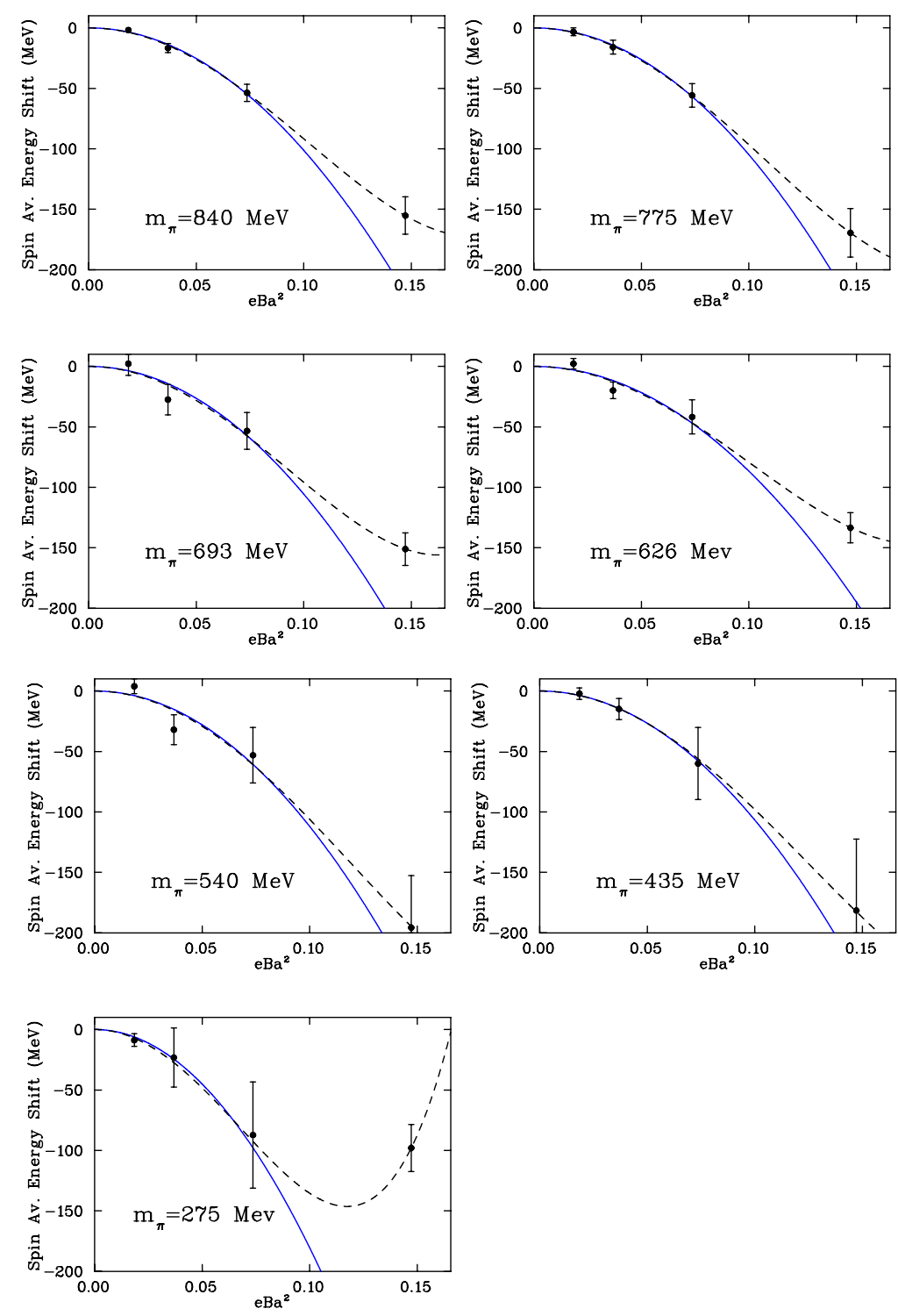

Figure A.19: Fits of the quenched neutron spin-averaged effective energy shift against the field at each quark mass. The solid line is a 1 parameter fit to the first three points, the dashed line is a 2 parameter fit to all 4 points. 